\title{
IDENTIFYING AND REDUCING IN-PLANE AND OUT-OF-PLANE ARTIFACTS IN PhotoACoustic IMAging
}

Ho Nhu Y Nguyen 


\section{Graduation Committee:}

Chairman/secretary: Prof. dr. J.L. Herek (University of Twente)

Supervisor: $\quad$ Prof. dr. ir. W. Steenbergen (University of Twente)

Committee Members: Prof. dr. S. Manohar (University of Twente)

Prof. dr. ir. R.M. Verdaasdonk (University of Twente)

Prof. dr. ing. G. Schmitz (Ruhr-University Bochum)

Dr. ir. R.G.P. Lopata (Eindhoven University of Technology)

Dr. M.K.A. Singh (Cyberdyne Inc.)

The work presented in this thesis has received funding from the European Union's Horizon 2020 research and innovation programme under grant agreement $\mathrm{n}^{\circ} 731771$.

The research was carried out at the Biomedical Photonic Imaging group, Faculty of Science and Technology, University of Twente.

Cover design: Ho Nhu Y Nguyen

Printed by: Gildeprint

ISBN: $\quad$ 978-90-365-5080-2

DOI: $\quad$ https://doi.org/10.3990/1.9789036550802

Copyright (C) 2020 Ho Nhu Y Nguyen, The Netherlands. All rights reserved. No parts of this thesis may be reproduced, stored in a retrieval system or transmitted in any form or by any means without permission of the author. Alle rechten voorbehouden. Niets uit deze uitgave mag worden vermenigvuldigd, in enige vorm of op enige wijze, zonder voorafgaande schriftelijke toestemming van de auteur. 


\title{
IDENTIFYING AND REDUCING IN-PLANE AND OUT-OF- PLANE ARTIFACTS IN PHOTOACOUSTIC IMAGING
}

\section{DISSERTATION}

\author{
to obtain \\ the degree of doctor at the University of Twente, \\ on the authority of the rector magnificus, \\ Prof. dr. ir. A. VELDKAMP, \\ on account of the decision of the Doctorate Board, \\ to be publicly defended \\ on Friday the $29^{\text {th }}$ of January 2021 at 14:45 hours
}

by

Ho Nhu Y Nguyen

born on the $16^{\text {th }}$ of April 1990

in Nghe An, Vietnam 
This dissertation has been approved by:

Supervisor

Prof. dr. ir. W. Steenbergen 


\section{Contents}

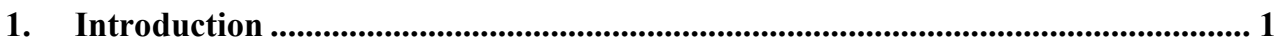

1.1. Photoacoustic imaging toward clinical applications ................................. 1

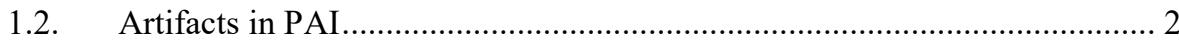

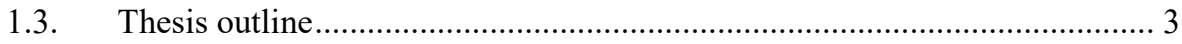

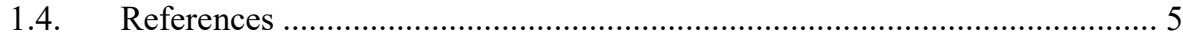

2. Recent Development of Technology and Application of Photoacoustic Molecular Imaging Toward Clinical Translation ......................................................................... 7

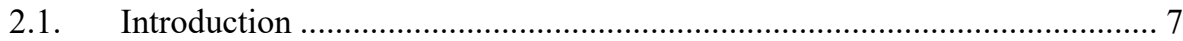

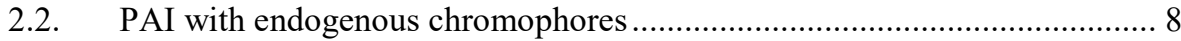

2.3. Photoacoustic molecular imaging with exogenous contrast agents ............13

2.4. Conclusion and future direction.............................................................. 19

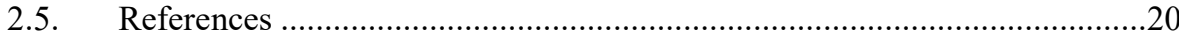

\section{Reflection Artifact Identification in Photoacoustic Imaging Using Multi-}

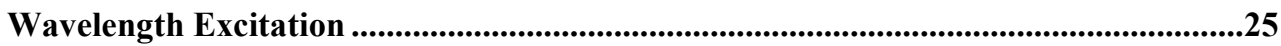

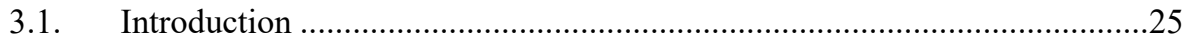

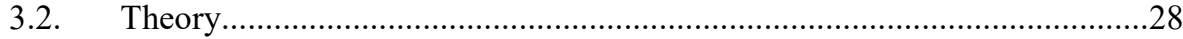

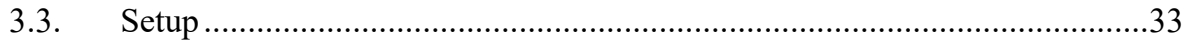

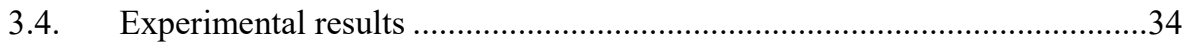

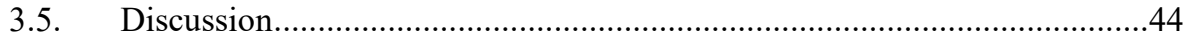

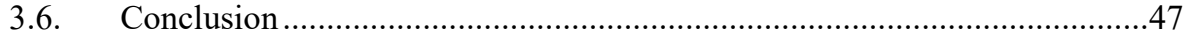

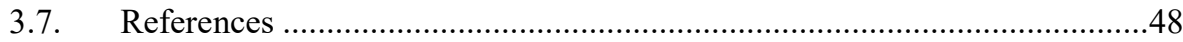


4. Reducing Artifacts in Photoacoustic Imaging by Using Multi-Wavelength Excitation and Transducer Displacement ...................................................................5

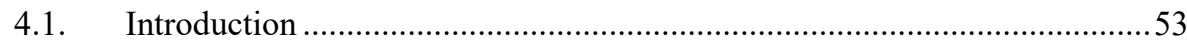

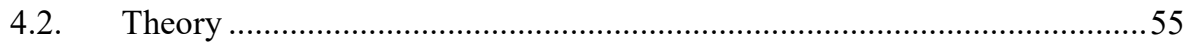

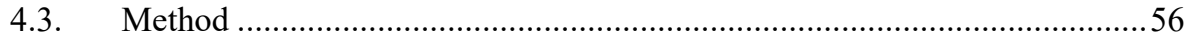

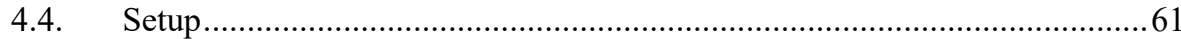

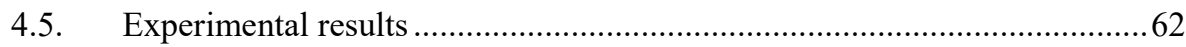

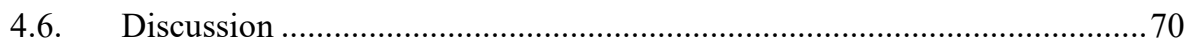

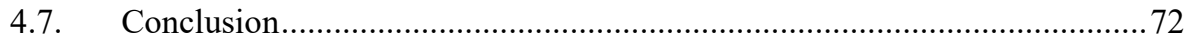

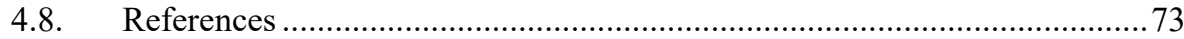

5. Three-dimensional View of Out-of-plane Artifacts in Photoacoustic Imaging

Using a Laser-integrated Linear-transducer-array Probe .........................................77

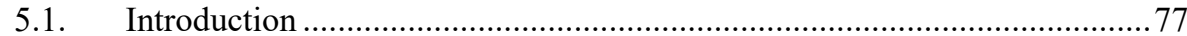

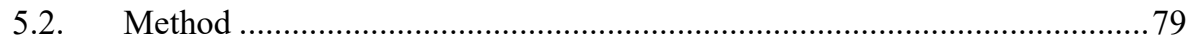

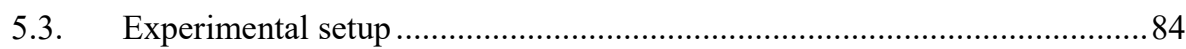

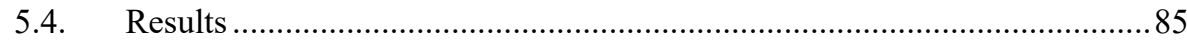

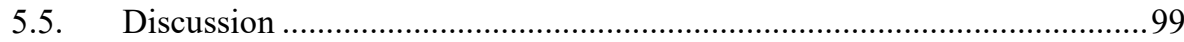

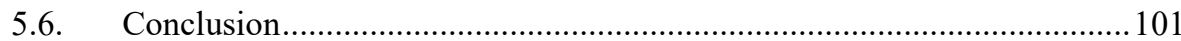

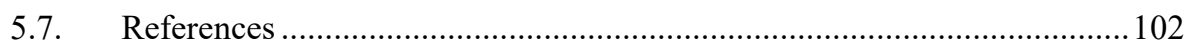

6. Feasibility of identifying reflection artifacts in photoacoustic imaging using two-

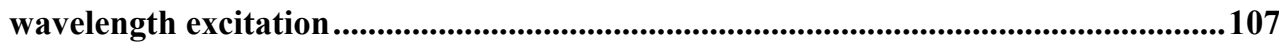

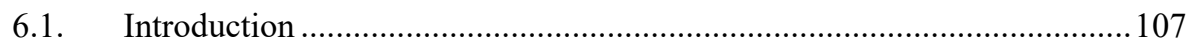

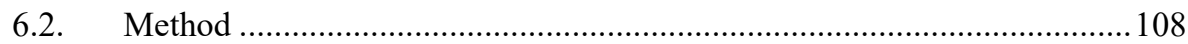

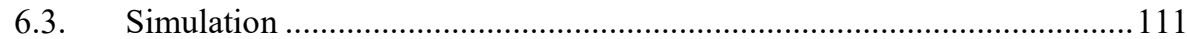

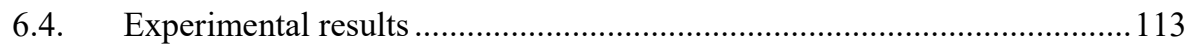

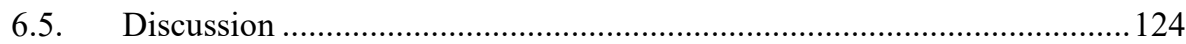

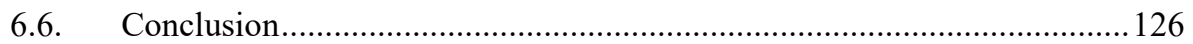

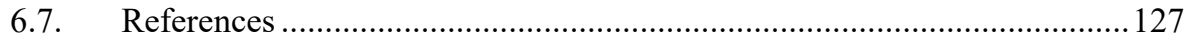




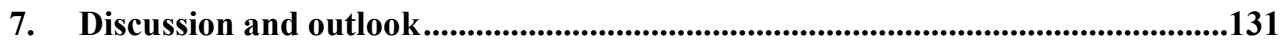

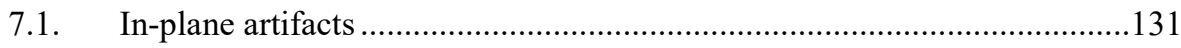

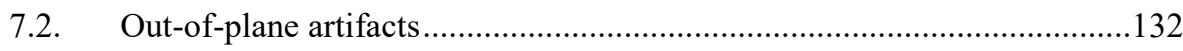

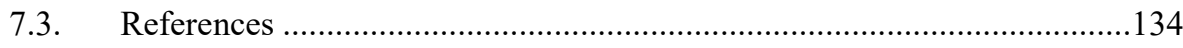

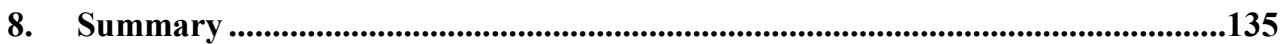

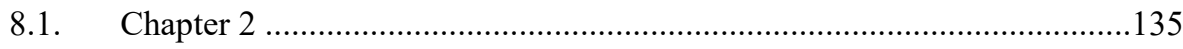

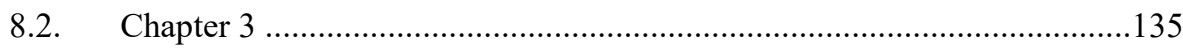

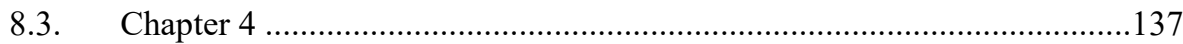

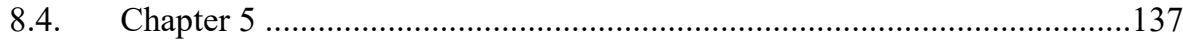

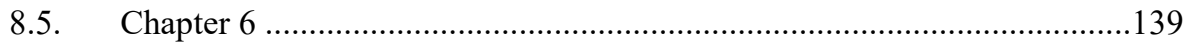

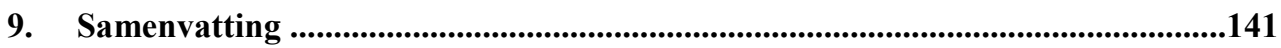

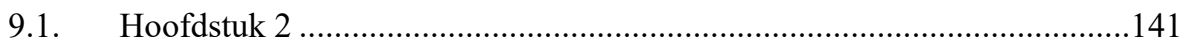

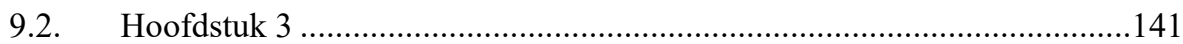

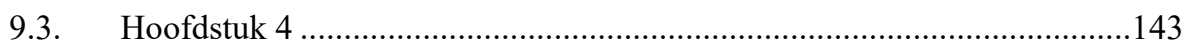

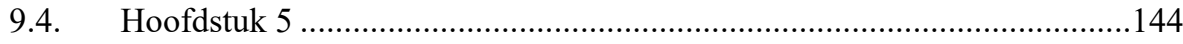

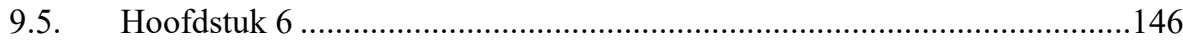

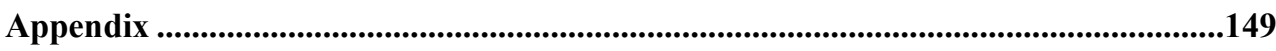

About the author

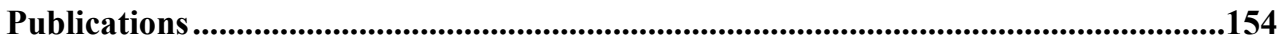

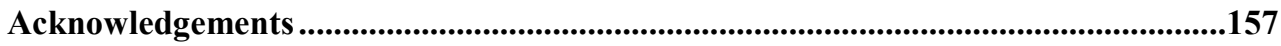





\section{Introduction}

This chapter presents a short overview of the current development of photoacoustic imaging. It also covers the main remaining drawbacks of this imaging technique especially the drawback that this thesis focuses on. At the end, it provides a guide through this thesis.

\subsection{Photoacoustic imaging toward clinical applications}

Photoacoustic imaging (PAI) is an imaging technique which is based on the photoacoustic (PA) effect. In this effect, ultrasound (US) waves are generated as a result of materials absorbing short pulsed light. In tissue, the US waves experience order of magnitude less scattering than light, PAI therefore provides images with clinically sufficient imaging depth, high resolution, and optical contrast.

Over the past few years, numerous studies on PAI have been reported as an effort to demonstrate the capability of this imaging technique in clinical applications [1-3]. PA images provide localized information of chromophores such as hemoglobin, melanin and lipids helping diagnose various diseases in early stages [4-7]. Toi et al. [6] showed that PAI can visualize angiogenesis in breasts, a hallmark of tumor in the early stage while Heijblom et al. [4] could visualize breast lesions with a higher contrast on PA images than on X-ray mammography. Van den Berg et al. [7] and Jo et al. [5] demonstrated the feasibility of PAI in visualizing inflamed finger joints in rheumatoid arthritis patients.

Though PAI, technically, holds great potential for clinical use, it still needs to be further improved mechanically and economically since a conventional PAI system is bulky and expensive. To reduce the size and the cost of a PAI system, current research focuses on using a handheld US probe with an integrated low cost light source such as diode lasers or lightemitting diodes [8-10]. However, due to the limited view angle of a handheld US probe, artifacts might occur leading to image misinterpretation. Identification, and if possible removal, of the artifacts is therefore highly needed. 


\subsection{Artifacts in PAI}

In PAI using a linear transducer array, acquired PA images are expected to show absorbers located inside the imaging plane of the array. However, the acoustic heterogeneity of the imaging object or absorbers located outside the imaging plane might cause artifacts (clutter) in the acquired images. Fig. 1.1 schematically shows situations in which such artifacts occur. There are four paths (numbered 1-4) of the US waves leading to artifacts. Paths 1 and 2 present US waves generated from an absorber located inside the imaging plane that are reflected back to the transducer array at an acoustic reflector located outside and inside the imaging plane respectively. An absorber located outside the imaging plane might also generate US waves that propagate directly (path 4) or indirectly (path 3) to the transducer array causing artifacts.

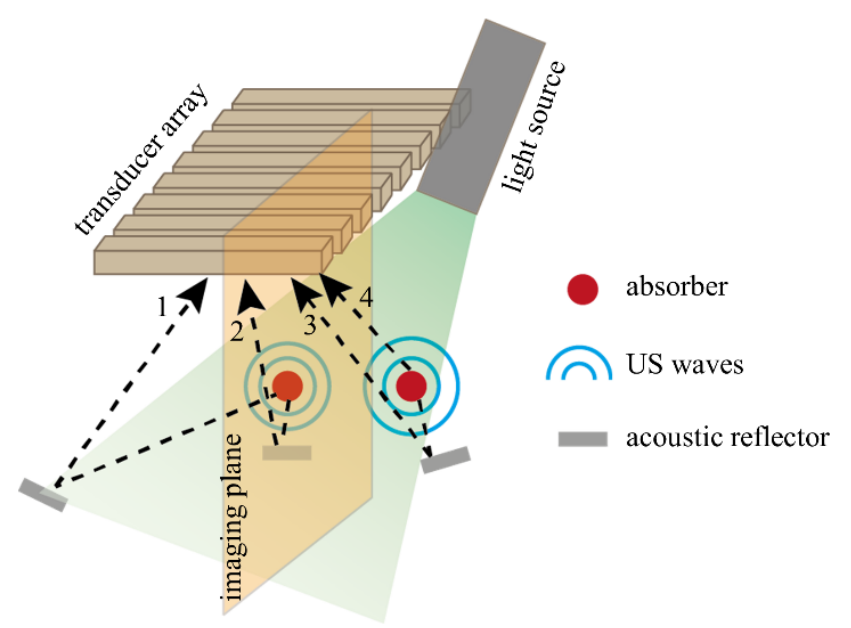

Fig. 1.1. Artifacts in PAI using a linear transducer array. 1-4 are possible paths of the US waves causing artifacts.

Artifacts occur in path 2 are called in-plane artifacts (IPAs) or reflection artifacts while the ones occur in paths 3 and 4 are called out-of-plane artifacts (OPAs). This thesis focuses on identifying and reducing these artifacts. Demonstrations in this thesis do not include artifacts in path 1. However, these artifacts have both properties of IPAs and OPAs, and the methods identifying IPAs and OPAs presented in this thesis would theoretically work for these artifacts as well. 
Several methods for reducing artifacts have been proposed [11-16]. Jaeger et al. [11, 12] de-correlated artifacts and image features by deforming the tissue. This method requires a well-trained user and sufficient deformation of tissue. Schwab et al. [13] used US plane waves to estimate the wave field of the IPAs. This method is computationally expensive as it requires numerous plane wave angles. Singh et al. $[14,16]$ introduced a method called PAFUSion (photoacoustic-guided focused ultrasound) mimicking the source of IPAs to identify them. However, a large number of additional US images is needed for this method to work and the reflectors need to be present in the imaging plane. Allman et al. [15] proposed a convolutional neural network to remove IPAs of point-like sources. The accuracy of this method might be negatively affected in in vivo imaging as it uses simulated data to train the network.

\subsection{Thesis outline}

This thesis focuses on developing new methods for identifying and removing IPAs and OPAs in PAI that offer new advantages to overcome the limitations of the existing methods. The proposed methods in this thesis are experimentally demonstrated in phantoms and in vivo using a US probe as well as a PA-US probe with integrated diode lasers. The demonstration takes advantage of the probe's cost and compactness over a conventional PAI system showing the potential of the methods for clinical use.

Chapter 2 is a review of the research over the past few years on photoacoustic molecular imaging exploiting endogenous chromophores and exogenous contrast agents. It focuses on spearheading translational efforts to clinics of this imaging technique.

Chapter 3 presents a method for identifying and removing in-plane artifacts (reflection artifacts) using multi-wavelength excitation. By imaging the sample with multiple wavelengths, the obtained spectral responses of the image features are used to compare between real image features and reflection artifacts. The method is demonstrated with phantom and in vivo experiments using a handheld PAI probe with 4 different wavelengths.

In Chapter 4, a method for identifying out-of-plane artifacts using axial transducer array displacement is presented. By axially displacing the transducer array, out-of-plane artifacts and in-plane image features are de-correlated revealing the out-of-plane artifacts. This 
method is then combined with the method for in-plane artifacts of Chapter 3 and experimentally demonstrated.

The method using axial transducer array displacement introduced in Chapter 4 is further exploited in Chapter 5 providing a three-dimensional (3D) view of out-of-plane artifacts. The sequence of PA images acquired during the displacement is back-projected elevationally to form a $3 \mathrm{D}$ image. The spatial sensitivity of the transducer array is taken into account of the $3 \mathrm{D}$ reconstruction.

Chapter 6 proposes a simplified version of the method presented in Chapter 3 when the number of wavelengths is reduced to 2. This Chapter demonstrates the feasibility of identifying reflection artifacts using 2 wavelengths while the original method requires at least 3 wavelengths. It also compares this simplified method with the original one showing new advantages of the simplified method.

Chapter 7 summarizes the work presented in this thesis and discusses the potential work in the future. 


\subsection{References}

[1] A. B. E. Attia, G. Balasundaram, M. Moothanchery, U. Dinish, R. Bi, V. Ntziachristos, and M. Olivo, "A review of clinical photoacoustic imaging: Current and future trends," Photoacoustics 16, 100144 (2019).

[2] I. Steinberg, D. M. Huland, O. Vermesh, H. E. Frostig, W. S. Tummers, and S. S. Gambhir, "Photoacoustic clinical imaging," Photoacoustics 14, 77-98 (2019).

[3] S. Manohar, and M. Dantuma, "Current and future trends in photoacoustic breast imaging," Photoacoustics 16, 100134 (2019).

[4] M. Heijblom, D. Piras, F. M. van den Engh, M. van der Schaaf, J. M. Klaase, W. Steenbergen, and S. Manohar, "The state of the art in breast imaging using the Twente Photoacoustic Mammoscope: results from 31 measurements on malignancies," European radiology 26(11), 3874-3887 (2016).

[5] J. Jo, G. Xu, M. Cao, A. Marquardt, S. Francis, G. Gandikota, and X. Wang, "A Functional Study of Human Inflammatory Arthritis Using Photoacoustic Imaging," Scientific reports 7(1), 15026 (2017).

[6] M. Toi, Y. Asao, Y. Matsumoto, H. Sekiguchi, A. Yoshikawa, M. Takada, M. Kataoka, T. Endo, N. Kawaguchi-Sakita, and M. Kawashima, "Visualization of tumor-related blood vessels in human breast by photoacoustic imaging system with a hemispherical detector array," Scientific Reports 7, (2017).

[7] P. J. van den Berg, K. Daoudi, H. J. B. Moens, and W. Steenbergen, "Feasibility of photoacoustic/ultrasound imaging of synovitis in finger joints using a point-of-care system," Photoacoustics 8, 8-14 (2017).

[8] M. K. A. Singh, W. Steenbergen, and S. Manohar, "Handheld probe-based dual mode ultrasound/photoacoustics for biomedical imaging," in Frontiers in Biophotonics for Translational Medicine(Springer, 2016), pp. 209-247.

[9] Q. Yao, Y. Ding, G. Liu, and L. Zeng, "Low-cost photoacoustic imaging systems based on laser diode and light-emitting diode excitation," Journal of Innovative Optical Health Sciences 10(04), 1730003 (2017). 
[10] K. Daoudi, P. Van Den Berg, O. Rabot, A. Kohl, S. Tisserand, P. Brands, and W. Steenbergen, "Handheld probe integrating laser diode and ultrasound transducer array for ultrasound/photoacoustic dual modality imaging," Optics express 22(21), 2636526374 (2014).

[11] M. Jaeger, L. Siegenthaler, M. Kitz, and M. Frenz, "Reduction of background in optoacoustic image sequences obtained under tissue deformation," Journal of biomedical optics 14(5), 054011 (2009).

[12] M. Jaeger, J. C. Bamber, and M. Frenz, "Clutter elimination for deep clinical optoacoustic imaging using localised vibration tagging (LOVIT)," Photoacoustics 1(2), 19-29 (2013).

[13] H.-M. Schwab, M. F. Beckmann, and G. Schmitz, "Photoacoustic clutter reduction by inversion of a linear scatter model using plane wave ultrasound measurements," Biomedical Optics Express 7(4), 1468-1478 (2016).

[14] M. K. A. Singh, and W. Steenbergen, "Photoacoustic-guided focused ultrasound (PAFUSion) for identifying reflection artifacts in photoacoustic imaging," Photoacoustics 3(4), 123-131 (2015).

[15] D. Allman, A. Reiter, and M. A. L. Bell, "Photoacoustic source detection and reflection artifact removal enabled by deep learning," IEEE transactions on medical imaging 37(6), 1464-1477 (2018).

[16] M. K. A. Singh, M. Jaeger, M. Frenz, and W. Steenbergen, "Photoacoustic reflection artifact reduction using photoacoustic-guided focused ultrasound: comparison between plane-wave and element-by-element synthetic backpropagation approach," Biomed. Opt. Express 8(4), 2245-2260 (2017). 


\title{
2. Recent Development of Technology
} and Application of Photoacoustic Molecular Imaging Toward Clinical

\section{Translation $^{1}$}

\begin{abstract}
The deep imaging capability and optical absorption contrast offered by photoacoustic imaging promote the use of this technology in clinical applications. By exploiting the optical absorption properties of endogenous chromophores such as hemoglobin and lipid, molecular information at a depth of a few centimeters can be unveiled. This information shows promise to reveal lesions indicating early stage of various human diseases, such as cancer and atherosclerosis. In addition, the use of exogenous contrast agents can further extend the capability of photoacoustic imaging in clinical diagnosis and treatment. In this review, the current state of the art and applications of photoacoustic molecular probes will be critically reviewed, as well as some spearheading translational efforts that have taken place over the past 5 years. Some of the most critical barriers to clinical translation of this novel technology will be discussed, and some thoughts will be given on future endeavors and pathways.
\end{abstract}

\subsection{Introduction}

Photoacoustic imaging (PAI) has been shown to be a promising medical imaging technology. This technique is based on the photoacoustic effect by which ultrasound waves are generated because of light absorption in the tissue's chromophores. Therefore, endogenous chromophores and exogenous contrast agents can be used to provide excellent

\footnotetext{
1 This chapter has been published as: J. Yu*, H. N. Y. Nguyen*, W. Steenbergen, and K. Kim, "Recent development of technology and application of photoacoustic molecular imaging toward clinical translation," Journal of Nuclear Medicine 59(8), 1202-1207 (2018).

* Contributed equally to this work
} 
contrast in photoacoustic molecular imaging. In a previous review, applications of photoacoustic molecular imaging of cancer in vivo using endogenous chromophores and exogenous contrast agents were discussed [1]. Over the past few years, the technology has been extensively applied to numerous human diseases. Diagnosis of various diseases at an early stage through screening, as well as monitoring of treatment, is possible using PAI with endogenous chromophores. In addition, novel approaches to the use of photoacoustic contrast agents have been significantly investigated to enhance contrast, targeting, and therapeutics over traditional probes for molecular theranostics. This review focuses on recent research on photoacoustic molecular imaging exploiting endogenous chromophores and exogenous contrast agents.

\subsection{PAI with endogenous chromophores}

Endogenous chromophores with significantly different absorption spectra produce high endogenous contrast in PAI. Specific wavelengths can be selected to obtain maximum contrast. Furthermore, PAI can take advantage of near-infrared light at wavelengths in the range 650-1,200 nm for deep imaging. Angiogenesis, the formation of new blood vessels, is one of the basic hallmarks of various diseases such as cancer [2]. Therefore, cancer can be revealed with PAI of hemoglobin, one of the major chromophores. As an example, Fig. 2.1 shows maximum-intensity-projection photoacoustic images of a 40-y-old woman's breasts after elimination of superficial blood vessels from the images. In this section, we focus on PAI uses that are close to becoming clinically applicable.

\subsubsection{Breast cancer}

Breast cancer is the leading cause of cancer death in women globally. In 2012, 0.5 million women worldwide died of breast cancer, accounting for $15 \%$ of all cancer deaths in women [3]. Because angiogenesis is the marker of cancer, imaging with light at wavelengths that are mainly absorbed by the chromophores deoxy- and oxyhemoglobin can be exploited to distinguish between malignant and normal breast tissue and to monitor therapy [2]. 

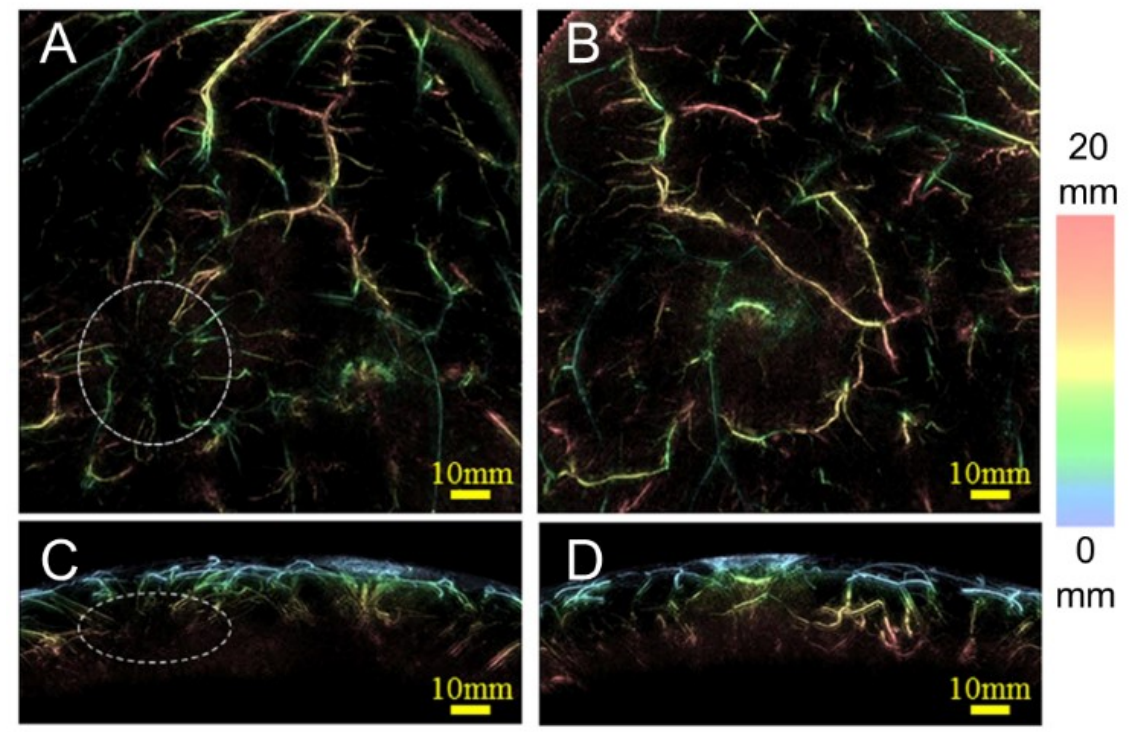

Fig. 2.1. PAI with depth color-coding of 40-y-old woman's breasts. Top view (A) and side view (C) show tumor and surrounding blood vessels (encircled), indicating angiogenesis. (B and D) Top view (B) and side view (D) of contralateral breast show no lesion. (Reproduced with permission of [4].)

Heijblom et al. used the Twente photoacoustic mammoscope to investigate breast cancer lesions in 31 patients [5], with 32 of 33 malignancies being visualized with high imaging contrast. On average, the lesions were seen with higher contrast on photoacoustic images than on x-ray mammography. Moreover, photoacoustic contrast was independent of mammographic breast density, whereas with $\mathrm{x}$-ray mammography there was a significant decrease in contrast in the low-density group compared with the highdensity group.

A new photoacoustic mammography system offering remarkably more morphologic and structural details of blood vessels than can be seen with MRI was reported by Toi et al. [4]. Additionally, combining these techniques allows visualization of both the tumor mass and its related vasculature in one image, shown in their study on 22 malignancies. By using 2 wavelengths (755 and $795 \mathrm{~nm}$ ) for imaging, the investigators could estimate the oxygen saturation of hemoglobin ( $\mathrm{sO} 2$ ). This information would give insight on the tumor microenvironment and facilitate monitoring of treatment such as chemotherapy and antiHER2 treatment. 


\subsubsection{Crohn disease}

Crohn disease is an inflammatory bowel disease often leading to severe injuries or even death. The inflammation can be revealed by measuring hemoglobin and oxygenated hemoglobin content in the intestinal wall. Knieling et al. proposed a noninvasive approach to distinguish active and nonactive Crohn disease by determining the hemoglobin level in the intestinal wall with multispectral photoacoustic tomography [6]. They performed multispectral photoacoustic tomography on 108 Crohn disease patients with 6 wavelengths and extracted total oxygenated and deoxygenated hemoglobin and $\mathrm{sO} 2$ in the intestinal wall. They found significant differences between active and nonactive Crohn disease for all multispectral photoacoustic tomography values excluding $\mathrm{sO} 2$ when using endoscopy and histologic characteristics as references.

\subsubsection{Rheumatoid arthritis}

Rheumatoid arthritis is a chronic inflammatory disease that causes progressive destruction of affected joints leading to severe disability and even death. Conventional radiography is the technique currently used for detection of joint damage. However, radiography cannot detect the disease in the early stage [7]. PAI, therefore, can be exploited because hypervascularization and angiogenesis are the hallmarks of the early stage.

van den Berg et al. observed large differences in photoacoustic images of inflamed, healthy, and noninflamed proximal interphalangeal finger joints [8]. Fig. 2.2 represents their setup and some results. They used a handheld probe with cost-efficient integrated diode lasers (Fig. 2.2A) and a compact PAI system for imaging finger joints (Fig. 2.2B). A larger number of high-photoacoustic pixels (indicating the presence of blood) was observed in the photoacoustic images of inflamed joints (Fig. 2.2C) than in the images of noninflamed joints (Fig. 2.2D). Fig. 2.2E shows their quantification study on the finger joints of 7 healthy volunteers and 10 rheumatoid arthritis patients. It appeared that inflamed joints have significantly higher image values than noninflamed joints. 

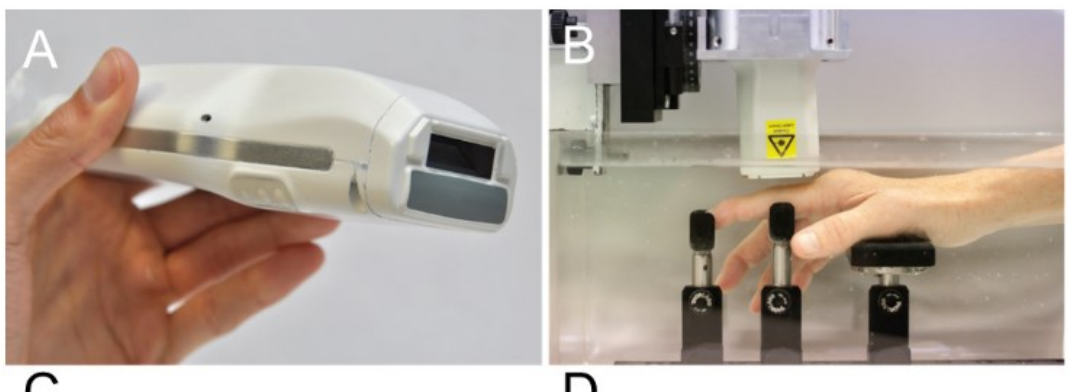

C

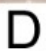

PA/US images (low and high PA amplitudes)

PA/US images (only high PA amplitudes)
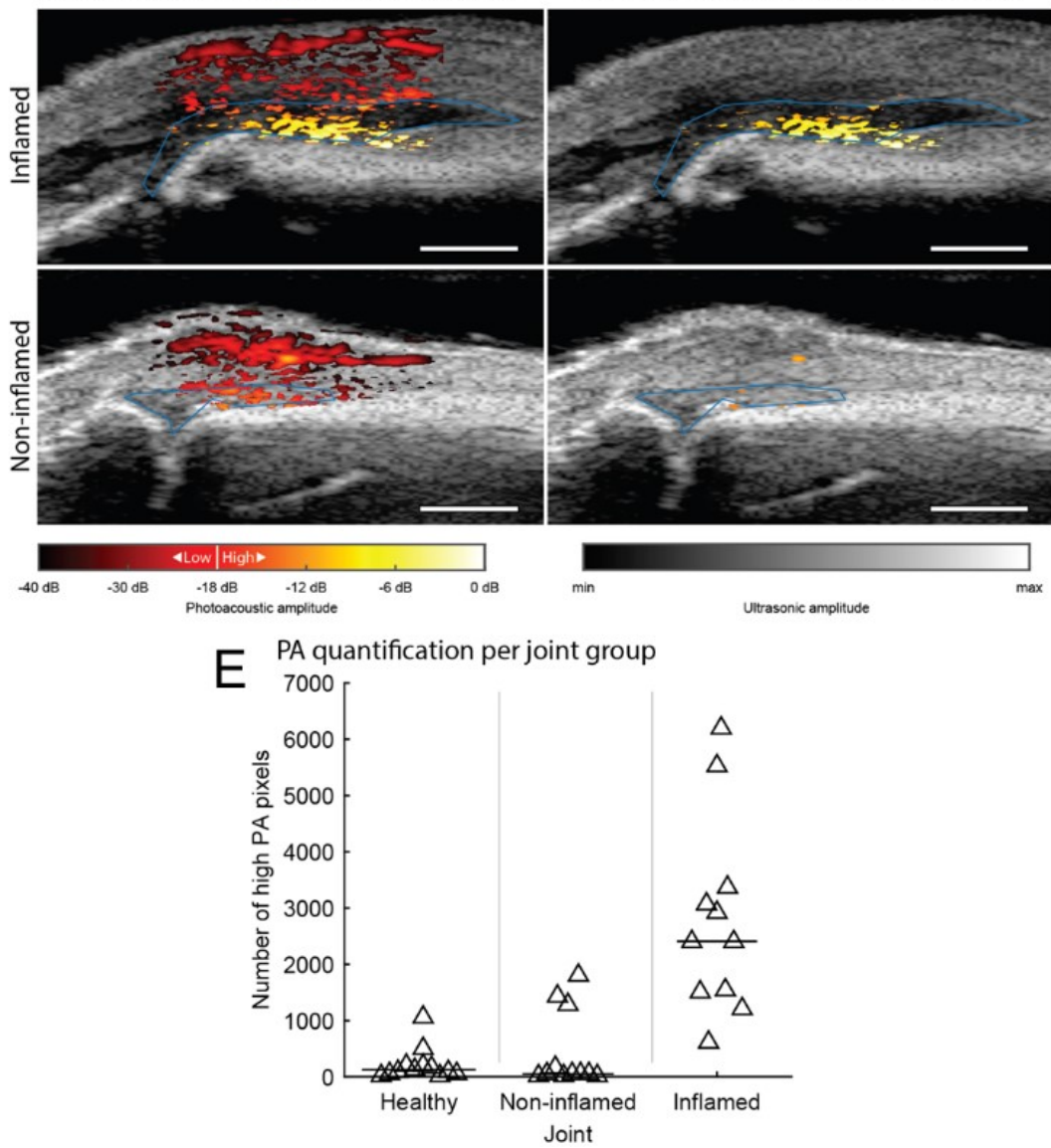

Fig. 2.2. (A) Handheld probe with integrated diode lasers. (B) Finger joint PAI setup. (C) Combined photoacoustic and ultrasound images of inflamed and noninflamed finger joints. (D) Processed images with only high photoacoustic amplitudes. (E) Photoacoustic quantification study on healthy volunteers and rheumatoid arthritis patients. PA 5 photoacoustic; US 5 ultrasound. (Reproduced with permission of [8].) 
Jo et al. enhanced van den Berg et al.'s observation with their clinical research on rheumatoid arthritis patients [8,9]. With a photoacoustic-ultrasound dual-imaging system, they could evaluate the increased hemoglobin content, exposing hyperemia, in arthritic joints over normal joints. In addition, they performed PAI using 2 wavelengths (576 and $584 \mathrm{~nm}$ ) to quantify $\mathrm{sO} 2$ in each joint. Their results showed a recognizable decrease in $\mathrm{sO} 2$, representing hypoxia, in arthritic joints compared with normal joints. Another report from this group showed the potential of using PAI to monitor the treatment process of arthritic joints [10].

\subsubsection{Psoriasis}

Psoriasis is an incurable inflammatory skin disease. Many noninvasive imaging techniques have been proposed to facilitate the study of psoriatic skins. However, they are limited by the imaging depth, contrast, or resolution [11, 12]. Aguirre et al. presented an ultra-broadband raster-scan photoacoustic mesoscopy system that could visualize the skin up to subdermis layer with a lateral resolution of about $20 \mathrm{~mm}$ [12]. Ultra-broadband rasterscan photoacoustic mesoscopy implemented in a hand-held probe allowed them to study vascular and melanin structures in healthy and psoriatic patients, reflecting the clinical potential of the technique. Images from 6 psoriasis patients showed that the blood volume per skin surface, the fractal number of the vasculature, and the epidermal thickness were all higher in psoriatic skin than in healthy skin. In addition, developing ultra-fast lasers will enable multispectral measurements for ultra-broadband raster-scan photoacoustic mesoscopy that could enhance the skin examination by showing oxygenation and differentiation of the melanin contributions.

\subsubsection{Prostate Cancer}

Prostate cancer is the second most common male cancer, close behind lung cancer. In 2012, 1.1 million men had prostate cancer, representing $15 \%$ of all cancers in men during that year [3]. Removing the tumor without affecting the nearby neurovascular bundles required for sexual potency is the goal of radical prostatectomy. Success strongly depends on the surgeon's experience. In addition, screening for angiogenesis can be used to detect the tumor. For those purposes, hemoglobin was exploited as an endogenous chromophore by Horiguchi et al. [13] and Ishihara et al. with a PAI system combining photoacoustic and 
ultrasound imaging [14]. They were able to visualize the periprostatic microvasculature and a photoacoustic signal pattern indicating cancer. Their handheld probe with real-time imaging shows promise for assisting surgeons during radical prostatectomy.

\subsubsection{Atherosclerotic plaque}

Vulnerable atherosclerotic plaque can lead to cardiovascular events, which are the leading cause of death globally [15]. Inside arteries, substances such as fat and cholesterol from the blood can accumulate in plaques. Lipids, therefore, are used as an endogenous contrast agent to reveal the plaque's vulnerability. Imaging lipid, however, is complicated by the blood's strong absorption. Wavelengths of maximum absorption by lipids are around 1,200 and $1,700 \mathrm{~nm}$.

Not all lipids indicate plaques. Jansen et al. could differentiate between plaque lipids and periadventitial lipids, which can be found in the wall of a normal artery $[16,17]$. They showed that these types of lipids have slightly different absorption spectra. Using a combined intravascular photoacoustic and ultrasound imaging system, with multiple wavelengths near 1,200 and $1,700 \mathrm{~nm}$, they were able to distinguish periadventitial lipids and lipids in the plaque in human coronary arteries ex vivo. Catheter-based intravascular photoacousticintravascular ultrasound imaging systems can display images in real time, enhancing this technique's advantages for clinical applications [18].

\subsection{Photoacoustic molecular imaging with exogenous contrast agents}

Exogenous photoacoustic contrast agents have been extensively studied in the past few years to provide molecular information on diseases with enhanced contrast [1]. Clinically approved fluorescent agents with low quantum yields such as sodium fluorescein, IRDye $800 \mathrm{CW}$, methylene blue, and indocyanine green have been repurposed for contrast-enhanced PAI in preclinical studies, but only one clinical study has been reported so far in which methylene blue was used to photoacoustically detect sentinel lymph node in breast cancer [19]. Besides these dyes, there have been continuous collective efforts to improve overall photoacoustic contrast, imaging depth, and specificity to the biomarkers, which would extend the capability of PAI for better diagnosis and treatments and might foster clinical translation. In this section, recent approaches to developing photoacoustic theranostic agents will be discussed. 


\subsubsection{Exogenous contrast agents for contrast-enhanced, deep-tissue, and multimodal PAI}

Although ultrasonically triggered phase-transition droplets as ultrasound contrast agent were discussed in the previous review, in this review optically triggered phase transition droplets as a promising photoacoustic contrast agent with superior efficiency are discussed $[1,20,21]$. Most photoacoustic contrast agents generate signals through thermal expansion, which is known to have relatively low photoacoustic conversion efficiency [21]. The perfluorocarbon family is often used to form the droplet core for its low bulk boiling temperature, such as perfluoropentane (boiling point, $29^{\circ} \mathrm{C}$ ). Once the liquid core is encapsulated by a thin layer of lipid, polymer, or protein, the vaporization temperature increases because of the increased Laplace pressure so that it maintains the liquid at physiologic temperature [22]. These shelled droplets contain chromophores that increase temperature locally on light absorption (Fig. 2.3A and B). The resulting rapid volume expansion of the shelledconstruct generates strong signals, providing significantly enhanced contrast compared with thermal expansion-based photoacoustic signals (Fig. 2.3B and C) $[20,22]$. With therapeutics inside and targeting molecules on the surface, these droplets can serve as disease-specific theranostic molecular probes (Fig. 2.3A). Furthermore, activated droplets that turn into gas bubbles can be used for contrast-enhanced ultrasound imaging. In this way, these droplets serve as dual-mode contrast agents for combined photoacoustic and ultrasound imaging [20, 23]. Recently, some efforts were initiated to unveil underlying physics of optically triggered droplets using theoretic models and experimental observations. These observations and findings so far may lead to the design of droplets with optimal parameters for in vivo use, which include a balance between photoacoustic conversion efficiency and safety, and may provide a strong motivation to further develop droplets and evaluate them for use as a theranostic nanoplatform conjugated with targeting and therapeutic molecules (Fig. 2.3D) [20, 22, 24]. 

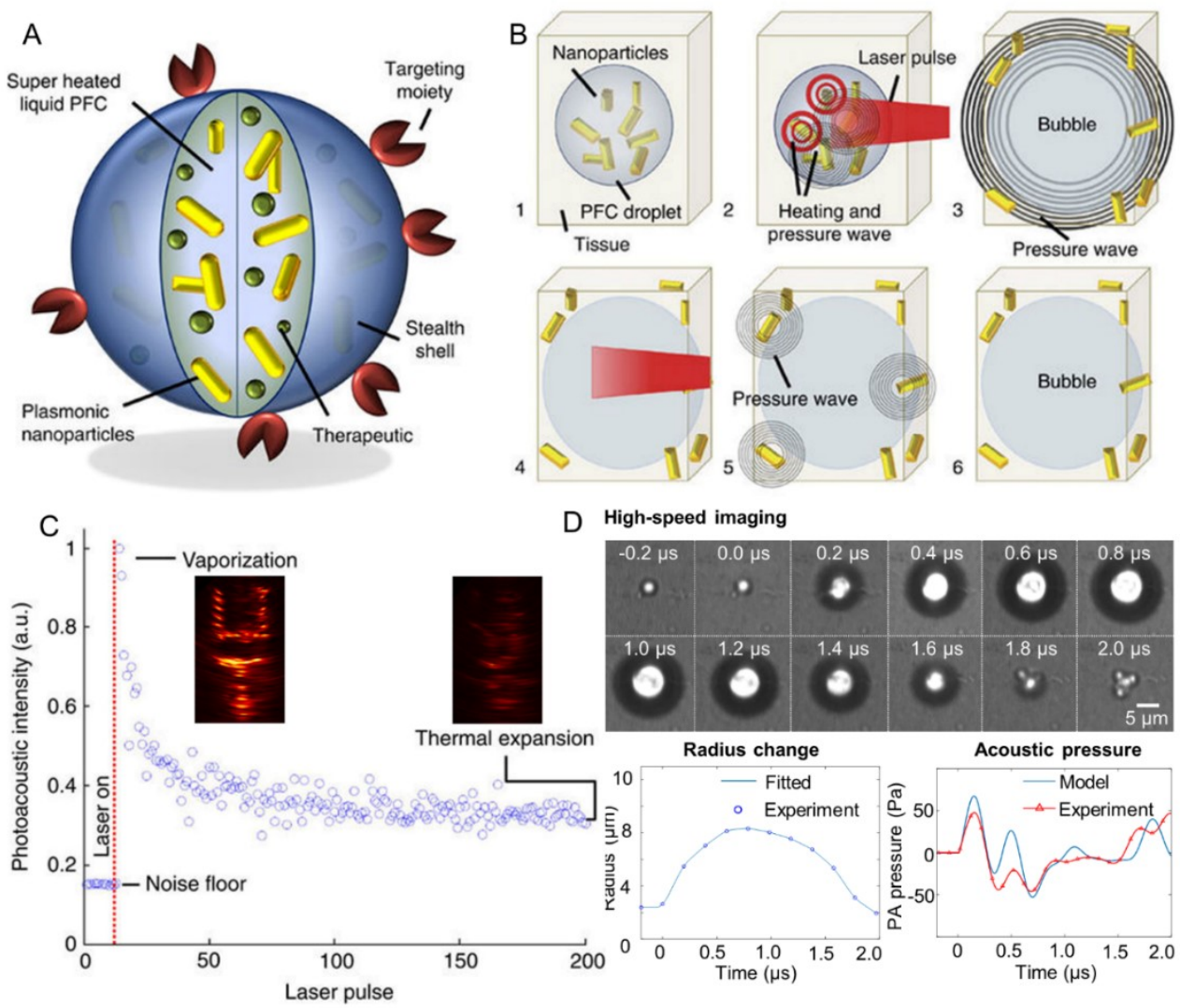

High-speed imaging
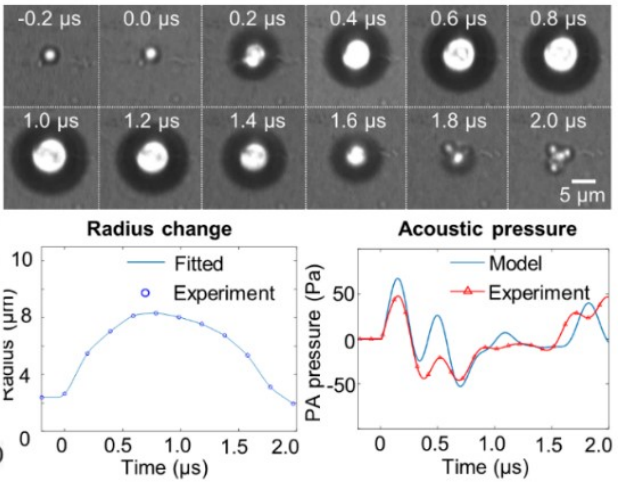

Fig. 2.3. (A) Conceptual structure of optically triggered phase transition droplet. (B) Illustration of optical droplet vaporization. (C) Photoacoustic signal enhancement using vaporization compared with thermal expansion. (D) Comparison between theoretic model and concurrent high-speed imaging and acoustic measurements of optical droplet vaporization. a.u. 5 arbitrary units; PFC 5 perfluorocarbon. (Reproduced with permission of [20,22].)

Relatively deep imaging is a key advantage of PAI over conventional optical imaging technologies. To maximize PAI depth, Zhou et al. suggested the use of phosphorus phthalocyanine with a long wavelength absorption beyond 1,000 $\mathrm{nm}$ to reduce light scattering, enhancing imaging depth. They successfully demonstrated PAI at depths of 11 $\mathrm{cm}$ in chicken breast tissue and $5 \mathrm{~cm}$ through human arms [25]. Also, surfactant-stripped naphthalocyanines developed by Zhang et al. were used for multimodal (ultrasound, photoacoustic, and PET) functional imaging in the gastrointestinal tract of a mouse [26]. 


\subsubsection{Molecular targeting approach for PAI}

Passive targeting relevant to oncology is based on size-dependent accumulation in solid tumors because of enhanced permeability and retention caused by leaky vasculature and poor lymphatic drainage in tumors. For active targeting, identifying the biomarkers that should be overexpressed on the target is required. As a targeting ligand, several structures are used, including small molecules, peptide/adhirons, Affibody, aptamer, and antibody/protein [27]. Some efforts were made to use these targeting ligands to achieve a molecular PAI.

Zhang et al. engineered a cystineknot peptide, $\mathrm{R}_{0} 1$, labeled with Atto740 dye and evaluated it using $\alpha_{\mathrm{v}} \beta_{6}$-positive (A431) and $\alpha_{\mathrm{v}} \beta_{6}$-negative (293T) tumors in mice [28]. Significant photoacoustic signal enhancement was found in A431 tumors over $4 \mathrm{~h}$ after injection (Fig. 2.4A-C). Levi et al. showed that their developed peptide, AA3G-740, successfully binds to gastrin-releasing peptide receptor in mouse prostate cancer, improving photoacoustic signal almost 2-fold compared with the control agent [29]. Sano et al. suggested an antiepidermal growth factor receptor monoclonal antibody labeled with indocyanine green to target cancer associated with epidermal growth factor receptor. The dye highly accumulated on epidermal growth factor receptor-positive (A431 and MDA-MB468) compared with -negative tumors (T47D) [30]. These technologies to simultaneously image both vascular networks by endogenous contrast and molecular features by exogenous contrast will enable molecular PAI for diagnosis and photoacoustic guided biopsy.

\subsubsection{Laser-activated therapy with guidance of PAI}

In recent years, PAI was used for guiding laser-activated therapy, photothermal therapy, and photodynamic therapy in preclinical research. Functional and molecular features of tissue shown by PAI, such as vasculature, hemodynamics, functional connectivity, melanoma, and temperature, have been used for monitoring treatment efficacy [31]. In addition, PAI has potential for monitoring drug distribution and concentration [31].

Photothermal therapy is localized thermal ablation using photothermal-conversion agents. Because the laser source can be shared, the synergetic ability for concurrent imaging and therapy has potential to monitor the accumulation of agents on-site during photothermal therapy. Zhang et al. successfully demonstrated treatment of a mouse tumor using terrylenediimide-based agents under PAI guidance [32]. The agents showed photothermal 
conversion efficiency of up to $40 \%$, with peak absorption at $640-690 \mathrm{~nm}$, which is well suited for both photothermal therapy and PAI. Muhanna et al. showed photothermal therapy with porphysomes, organic nanoparticles developed for fluorescence and photoacoustic dualmodality imaging [33]. Porphysomeenabled photothermal therapy allowed for PAImonitored target-specific ablation of buccal and tongue carcinomas in hamster, and its progress was monitored by PAI (Fig. 2.4D and E).

Photosensitizers for photodynamic therapy require oxygen to generate toxic singlet oxygen that kills the target cells. Near-infrared light is desired for laser activation at sufficient depths while also allowing for deep PAI. Yu et al. demonstrated PAI with a clinical photosensitizer, verteporfin, on a mouse tumor [34]. Ho et al. evaluated the photoacoustic efficacy of 5 different photosensitizers [35]. Zinc phthalocyanine exhibited the most efficient photoacoustic response, and in vivo feasibility was shown using a mouse tumor model. 


\section{Targeted PAl with antibody}

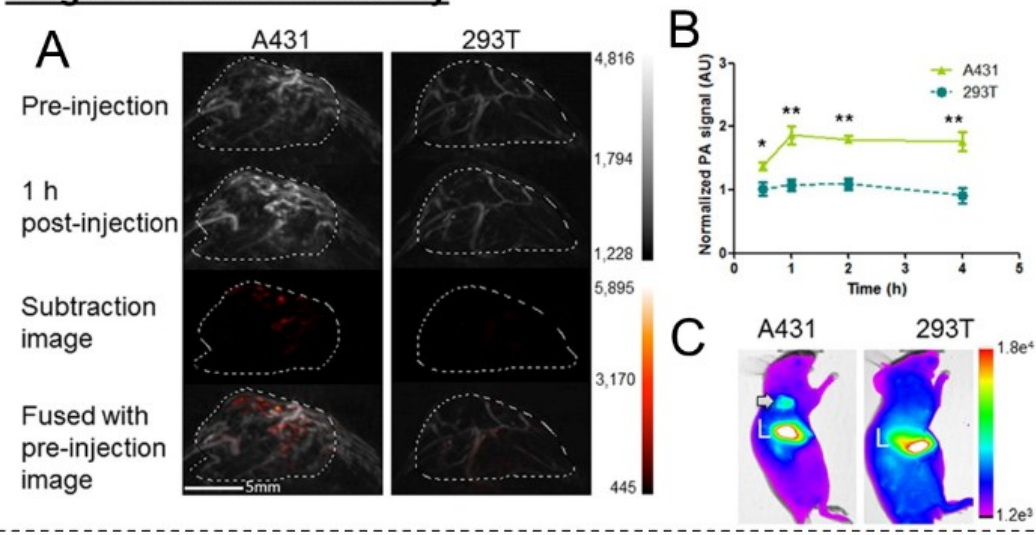

PAl guided PTT using porphysomes

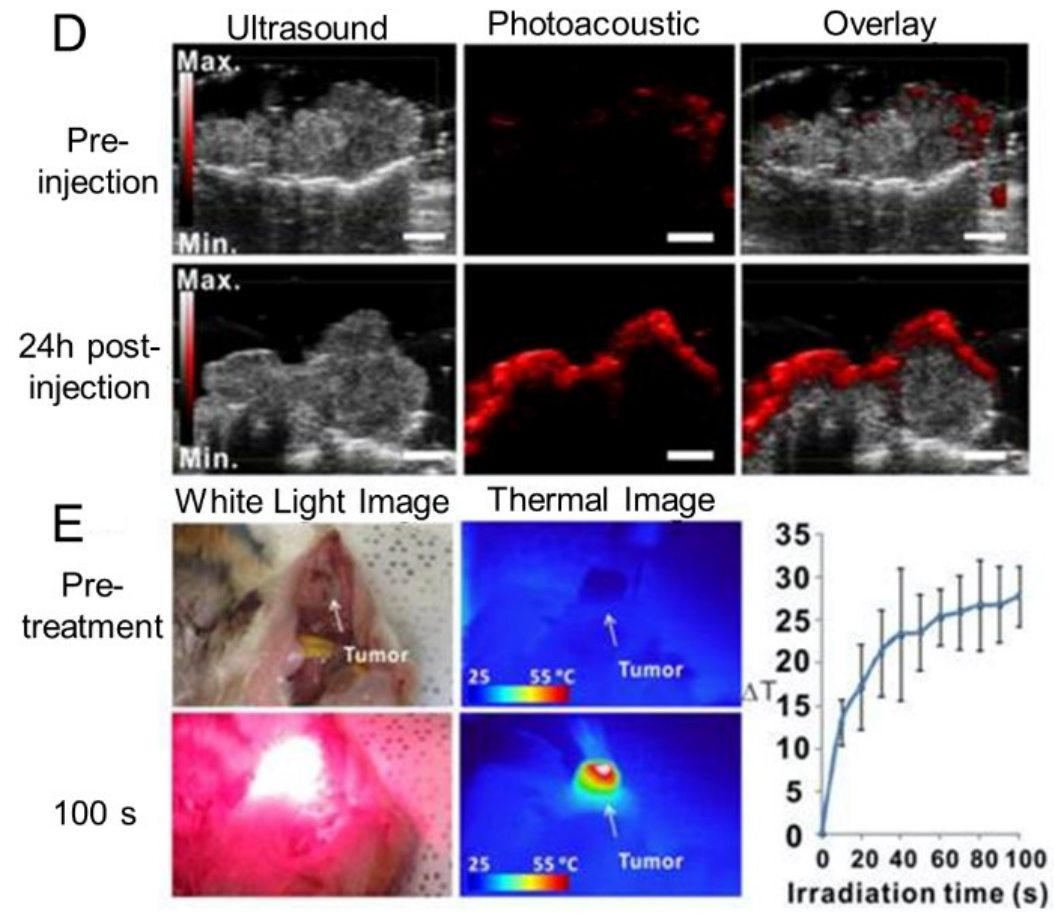

Fig. 2.4. (A and B) PAI of A431 tumor with antibody targeting of $\alpha_{v} \beta_{6}$ integrin. (C) Fluorescence imaging at $1 \mathrm{~h}$ after injection of A740-R01. Arrow points to A431 tumor. L 5 liver. (D) Photoacoustic detection of hamster oral tumor at $24 \mathrm{~h}$ after injection of porphysomes. (E) Bright-field and thermal image of tumor before and $100 \mathrm{~s}$ after porphysome-enabled photothermal therapy. a.u. 5 arbitrary units; PA 5 photoacoustic; PTT 5 photothermal therapy. (Reproduced with permission of $[28,33]$.) 


\subsection{Conclusion and future direction}

PAI with advanced multifunctional theranostic agents has great potential as a noninvasive and nonionization biomedical molecular imaging and treatment tool. In addition to advanced molecular probes, clinical acceptance will increase with the advent of faster, more compact, affordable, and clinically friendly systems. One big barrier is the laser. A Qswitched solid-state laser for a typical PAI system is bulky and costly, and the wavelengthtuning speed on the optical-parametric-oscillator is too slow to achieve real-time multispectral PAI. Recently, Schwarz et al. [36] introduced a PAI system with a high repetition rate $(100 \mathrm{~Hz})$ and a fast wavelength-tuning speed $(10 \mathrm{~ms})$. In addition, there have been efforts to develop affordable PAI systems, such as replacing the bulky solidstate lasers with diode lasers or light-emitting diodes and integrating the light source and the detector into a handheld probe $[37,38]$. In efforts to make PAI more suitable for freehand scanning in a clinical environment, a concave-mirror-shaped device attached to the ultrasound probe was proposed to not only protect an operator and a patient from unwanted exposure to the reflected laser light but also improve the signal-to-noise-ratio for deep tissue imaging [34]. Exogenous photoacoustic contrast agents also should be further evaluated under biologically relevant conditions to assess bioeffects and improve binding or treatment efficiency in situ. All these approaches will bring PAI closer to clinical application. Continued efforts to develop clinically friendly systems and novel multifunctional photoacoustic contrast agents and to validate them in human subjects will accelerate the clinical introduction of photoacoustic technologies. 


\subsection{References}

[1] K. E. Wilson, T. Y. Wang, and J. K. Willmann, "Acoustic and photoacoustic molecular imaging of cancer," Journal of Nuclear Medicine 54(11), 1851-1854 (2013).

[2] D. Hanahan, and R. A. Weinberg, "Hallmarks of cancer: the next generation," cell 144(5), 646-674 (2011).

[3] C. P. Wild, and B. W. Stewart, World Cancer Report 2014 (World Health Organization, 2014).

[4] M. Toi, Y. Asao, Y. Matsumoto, H. Sekiguchi, A. Yoshikawa, M. Takada, M. Kataoka, T. Endo, N. Kawaguchi-Sakita, and M. Kawashima, "Visualization of tumor-related blood vessels in human breast by photoacoustic imaging system with a hemispherical detector array," Scientific Reports 7, (2017).

[5] M. Heijblom, D. Piras, F. M. van den Engh, M. van der Schaaf, J. M. Klaase, W. Steenbergen, and S. Manohar, "The state of the art in breast imaging using the Twente Photoacoustic Mammoscope: results from 31 measurements on malignancies," European radiology 26(11), 3874-3887 (2016).

[6] F. Knieling, C. Neufert, A. Hartmann, J. Claussen, A. Urich, C. Egger, M. Vetter, S. Fischer, L. Pfeifer, and A. Hagel, "Multispectral Optoacoustic Tomography for Assessment of Crohn's Disease Activity," New England Journal of Medicine 376(13), 1292-1294 (2017).

[7] M. Backhaus, T. Kamradt, D. Sandrock, D. Loreck, J. Fritz, K. Wolf, H. Raber, B. Hamm, G. R. Burmester, and M. Bollow, "Arthritis of the finger joints: a comprehensive approach comparing conventional radiography, scintigraphy, ultrasound, and contrast-enhanced magnetic resonance imaging," Arthritis \& Rheumatology 42(6), 1232-1245 (1999).

[8] P. J. van den Berg, K. Daoudi, H. J. B. Moens, and W. Steenbergen, "Feasibility of photoacoustic/ultrasound imaging of synovitis in finger joints using a point-of-care system," Photoacoustics 8, 8-14 (2017). 
[9] J. Jo, G. Xu, M. Cao, A. Marquardt, S. Francis, G. Gandikota, and X. Wang, "A Functional Study of Human Inflammatory Arthritis Using Photoacoustic Imaging," Scientific reports 7(1), 15026 (2017).

[10] J. R. Rajian, X. Shao, D. L. Chamberland, and X. Wang, "Characterization and treatment monitoring of inflammatory arthritis by photoacoustic imaging: a study on adjuvant-induced arthritis rat model," Biomedical optics express 4(6), 900-908 (2013).

[11] F. Lacarrubba, G. Pellacani, S. Gurgone, A. E. Verzì, and G. Micali, "Advances in noninvasive techniques as aids to the diagnosis and monitoring of therapeutic response in plaque psoriasis: a review," International journal of dermatology 54(6), 626-634 (2015).

[12] J. Aguirre, M. Schwarz, N. Garzorz, M. Omar, A. Buehler, K. Eyerich, and V. Ntziachristos, "Precision assessment of label-free psoriasis biomarkers with ultrabroadband optoacoustic mesoscopy," Nature Biomedical Engineering 1, 0068 (2017).

[13] A. Horiguchi, K. Tsujita, K. Irisawa, T. Kasamatsu, K. Hirota, M. Kawaguchi, M. Shinchi, K. Ito, T. Asano, and H. Shinmoto, "A pilot study of photoacoustic imaging system for improved real-time visualization of neurovascular bundle during radical prostatectomy," The Prostate 76(3), 307-315 (2016).

[14] M. Ishihara, M. Shinchib, A. Horiguchi, H. Shinmotoc, H. Tsudad, K. Irisawae, T. Wadae, and T. Asanob, "Possibility of transrectal photoacoustic imaging-guided biopsy for detection of prostate cancer," in Proc. of SPIE Vol (2017), pp. 100642U100641.

[15] W. H. Organization, "Cardiovascular diseases (CVDs)," http://www. who. int/mediacentre/factsheets/fs317/en/index. html, (2009).

[16] K. Jansen, M. Wu, A. F. van der Steen, and G. van Soest, "Lipid detection in atherosclerotic human coronaries by spectroscopic intravascular photoacoustic imaging," Optics express 21(18), 21472-21484 (2013).

[17] K. Jansen, M. Wu, A. F. van der Steen, and G. van Soest, "Photoacoustic imaging of human coronary atherosclerosis in two spectral bands," Photoacoustics 2(1), 12-20 (2014). 
[18] M. Wu, G. Springeling, M. Lovrak, F. Mastik, S. Iskander-Rizk, T. Wang, H. M. van Beusekom, A. van der Steen, and G. Van Soest, "Real-time volumetric lipid imaging in vivo by intravascular photoacoustics at 20 frames per second," Biomedical optics express 8(2), 943-953 (2017).

[19] A. Garcia-Uribe, T. N. Erpelding, A. Krumholz, H. Ke, K. Maslov, C. Appleton, J. A. Margenthaler, and L. V. Wang, "Dual-modality photoacoustic and ultrasound imaging system for noninvasive sentinel lymph node detection in patients with breast cancer," Scientific reports 5, 15748 (2015).

[20] K. Wilson, K. Homan, and S. Emelianov, "Biomedical photoacoustics beyond thermal expansion using triggered nanodroplet vaporization for contrast-enhanced imaging," Nature communications 3(1), 1-10 (2012).

[21] A. C. Tam, "Applications of photoacoustic sensing techniques," Reviews of Modern Physics 58(2), 381 (1986).

[22] T. Feng, Q. Li, C. Zhang, G. Xu, L. J. Guo, J. Yuan, and X. Wang, "Characterizing cellular morphology by photoacoustic spectrum analysis with an ultra-broadband optical ultrasonic detector," Opt. Express 24(17), 19853-19862 (2016).

[23] H. Yoon, S. K. Yarmoska, A. S. Hannah, C. Yoon, K. A. Hallam, and S. Y. Emelianov, "Contrast-enhanced ultrasound imaging in vivo with laser-activated nanodroplets," Medical physics 44(7), 3444-3449 (2017).

[24] G. Lajoinie, E. Gelderblom, C. Chlon, M. Böhmer, W. Steenbergen, N. De Jong, S. Manohar, and M. Versluis, "Ultrafast vapourization dynamics of laser-activated polymeric microcapsules," Nature communications 5(1), 1-8 (2014).

[25] Y. Zhou, D. Wang, Y. Zhang, U. Chitgupi, J. Geng, Y. Wang, Y. Zhang, T. R. Cook, J. Xia, and J. F. Lovell, "A phosphorus phthalocyanine formulation with intense absorbance at $1000 \mathrm{~nm}$ for deep optical imaging," Theranostics 6(5), 688 (2016).

[26] Y. Zhang, M. Jeon, L. J. Rich, H. Hong, J. Geng, Y. Zhang, S. Shi, T. E. Barnhart, P. Alexandridis, and J. D. Huizinga, "Non-invasive multimodal functional imaging of the intestine with frozen micellar naphthalocyanines," Nature nanotechnology 9(8), 631 (2014). 
[27] J. Weber, P. C. Beard, and S. E. Bohndiek, "Contrast agents for molecular photoacoustic imaging," Nature methods 13(8), 639-650 (2016).

[28] C. Zhang, R. Kimura, L. Abou-Elkacem, J. Levi, L. Xu, and S. S. Gambhir, "A cystine knot peptide targeting integrin $\alpha v \beta 6$ for photoacoustic and fluorescence imaging of tumors in living subjects," Journal of Nuclear Medicine 57(10), 1629-1634 (2016).

[29] J. Levi, A. Sathirachinda, and S. S. Gambhir, "A high-affinity, high-stability photoacoustic agent for imaging gastrin-releasing peptide receptor in prostate cancer," Clinical Cancer Research 20(14), 3721-3729 (2014).

[30] K. Sano, M. Ohashi, K. Kanazaki, N. Ding, J. Deguchi, Y. Kanada, M. Ono, and H. Saji, "In vivo photoacoustic imaging of cancer using indocyanine green-labeled monoclonal antibody targeting the epidermal growth factor receptor," Biochemical and biophysical research communications 464(3), 820-825 (2015).

[31] J. Xia, C. Kim, and J. F Lovell, "Opportunities for photoacoustic-guided drug delivery," Current drug targets 16(6), 571-581 (2015).

[32] S. Zhang, W. Guo, J. Wei, C. Li, X.-J. Liang, and M. Yin, "Terrylenediimide-based intrinsic theranostic nanomedicines with high photothermal conversion efficiency for photoacoustic imaging-guided cancer therapy," ACS nano 11(4), 3797-3805 (2017).

[33] N. Muhanna, C. S. Jin, E. Huynh, H. Chan, Y. Qiu, W. Jiang, L. Cui, L. Burgess, M. K. Akens, and J. Chen, "Phototheranostic porphyrin nanoparticles enable visualization and targeted treatment of head and neck cancer in clinically relevant models," Theranostics 5(12), 1428 (2015).

[34] J. Yu, J. S. Schuman, J.-K. Lee, S.-G. Lee, J. H. Chang, and K. Kim, "A light illumination enhancement device for photoacoustic imaging: in vivo animal study," IEEE transactions on ultrasonics, ferroelectrics, and frequency control 64(8), 1205 1211 (2017).

[35] C. J. H. Ho, G. Balasundaram, W. Driessen, R. McLaren, C. L. Wong, U. Dinish, A. B. E. Attia, V. Ntziachristos, and M. Olivo, "Multifunctional photosensitizer-based contrast agents for photoacoustic imaging," Scientific reports 4(1), 1-6 (2014). 
[36] M. Schwarz, A. Buehler, J. Aguirre, and V. Ntziachristos, "Three-dimensional multispectral optoacoustic mesoscopy reveals melanin and blood oxygenation in human skin in vivo," Journal of biophotonics 9(1-2), 55-60 (2016).

[37] Q. Yao, Y. Ding, G. Liu, and L. Zeng, "Low-cost photoacoustic imaging systems based on laser diode and light-emitting diode excitation," Journal of Innovative Optical Health Sciences 10(04), 1730003 (2017).

[38] K. Daoudi, P. Van Den Berg, O. Rabot, A. Kohl, S. Tisserand, P. Brands, and W. Steenbergen, "Handheld probe integrating laser diode and ultrasound transducer array for ultrasound/photoacoustic dual modality imaging," Optics express 22(21), 2636526374 (2014). 


\title{
3. Reflection Artifact Identification in
} Photoacoustic Imaging Using MultiWavelength Excitation ${ }^{2}$

\begin{abstract}
Photoacoustic imaging has been a focus of research for clinical applications, owing to its ability for deep visualization with optical absorption contrast. However, there are various technical challenges remaining for this technique to find its place in clinics. One of the challenges is the occurrence of reflection artifacts. The reflection artifacts may lead to image misinterpretation. Here we propose a new method using multiple wavelengths for identifying and removing the reflection artifacts. By imaging the sample with multiple wavelengths, the spectral response of the features in the photoacoustic image is obtained. We assume that the spectral response of the reflection artifact is better correlated with the proper image feature of its corresponding absorber than with other features in the image. Based on this, the reflection artifacts can be identified and removed. Here we, experimentally demonstrated the potential of this method for real-time identification and correction of reflection artifacts in photoacoustic images in phantoms as well as in vivo using a handheld photoacoustic imaging probe.
\end{abstract}

\subsection{Introduction}

In the last decade, significant progress has been made for translating photoacoustic imaging (PAI) into clinics [1]. This technique uses the photoacoustic (PA) effect, where materials absorb short pulsed light and generate ultrasound (US) waves. The US waves can be detected using US transducers for reconstructing the absorbing structures. Since in tissue the US waves experience order of magnitude less scattering compared to light, much deeper

\footnotetext{
2 This chapter has been published as: H. N. Y. Nguyen, A. Hussain, and W. Steenbergen, "Reflection artifact identification in photoacoustic imaging using multi-wavelength excitation," Biomedical Optics Express 9(10), 4613-4630 (2018).
} 
information can be reconstructed compared to purely optical imaging techniques. Therefore, PAI provides optical absorption contrast and has the ability to image deeper than purely optical imaging techniques at ultrasonic resolution. Exploiting these properties, current research is focusing on investigating its clinical applications such as imaging of breast cancer $[2,3]$, rheumatoid arthritis [4, 5], and atherosclerosis [6]. Additionally, multispectral PAI strengthens the advantages of this technique for screening and monitoring human diseases, for instance by examining the oxygen saturation of hemoglobin $\left(\mathrm{sO}_{2}\right)$ in the lesion [3] or characterizing different tissues [6].

Recent research has focused on developing compact and affordable PAI systems. Integrating the laser source, especially a low cost laser source [7], into commercial handheld US probes for clinical use of PAI systems was proposed [7-12]. However, one of the major drawbacks of using a linear US transducer array is the occurrence of reflection artifacts (RAs) due to its limited view angle. As photoacoustically generated US waves propagate in all directions, the US waves propagating away from the US transducer array can be reflected towards the US transducer array by acoustic heterogeneities such as bone and tendon causing RAs in the acquired photoacoustic image. The RAs appear at larger depths than the real absorbers leading to misinterpretation of the acquired images. For clinical usage, real-time correction of the RAs in PAI is of fundamental importance.

RAs are also called in-plane artifacts, one of two types of artifacts (clutter) in PAI. The other type is out-of-plane artifact [13]. The term "plane" represents the imaging plane defined by the US transducer array. Since the laser beam excites a large volume, absorbers which are not in the imaging plane absorb the light and generate signals. If the out-of-plane sensitivity of the transducers is high enough, these absorbers appear in the acquired image resulting in out-of-plane artifacts (direct out-of-plane artifacts). If there is an acoustic reflector located underneath these out-of-plane absorbers, out-of-plane RAs (indirect out-ofplane artifacts) can be present in the acquired image [13]. In this work, we aim to tackle RAs (in-plane artifacts).

Several methods for reducing RAs have been presented [14-18]. Deformation Compensated Averaging (DCA) [14] employs tissue deformation for de-correlating the artifact by slightly palpating the tissue. This technique requires a well-trained person, sufficient deformation of tissue and works for easily deformable tissue. Localized vibration 26 
tagging (LOVIT) [15], introduced by Jaeger, uses a similar principle as DCA but using the acoustic radiation force (ARF) aimed at the artifact in the focal region of the ultrasonic beam instead of tissue palpation. This is a promising approach to overcome the disadvantages of DCA. However, it can only reduce artifacts based on the deformation of tissue in the US focal region. This limits the real-time capability and has safety challenges. Recently, LOVIT has been further improved by using multiple foci [19]. Another method exploits the acoustic tissue information by inversion of a linear scatter model using plane wave US measurements [16]. This method has to match PA and US measurements and requires numerous plane wave angles limiting itself to real-time performance. Schwab then introduced an advanced interpolation approach to significantly reduce the required number of plane waves in a linear scattering medium [20]. Allman introduced a convolutional neural network to remove RAs of point-like sources with high accuracy [18]. However, since the network is trained with simulated data, the accuracy might be negatively affected in in vivo situations.

Previously Singh introduced a method, photoacoustic-guided focused ultrasound (PAFUSion), using focused ultrasound or synthetic backpropagation to mimic PA sources and thus identify the RAs $[17,21,22]$. This method can efficiently reduce the RAs, however it has several limitations: mimicking the PA source is limited by the angular aperture of the US probe; numerous additional US images are needed, challenging real-time artifact reduction; the PA sources (skin, blood vessels) must be perpendicular to the imaging plane that requires demanding alignment effort; the PA signal from the source and the mimicked signal by US must match each other in terms of amplitude and frequency content which might negatively affect the accuracy of the method.

In this paper, we propose a new method where we exploit the use of multispectral PAI for identifying and removing RAs. Imaging with multiple wavelengths, PA spectral responses of the features in the acquired image can be obtained. Our method is based on the assumption that RAs are better correlated with the image features of their corresponding original absorbers than with other features, exposing the suspicious artifacts. In addition, RAs appear at larger depths and have weaker signals than the original image feature. Combining these findings can reveal the RAs and remove them.

To test the method, a handheld probe with integrated diode lasers was used for PAI. These diode lasers emit light at 4 wavelengths $(808,915,940$ and $980 \mathrm{~nm})$. We performed 
experiments in phantoms and in vivo. Results show that this is a promising method for correcting RAs, potentially in real-time.

\subsection{Theory}

\subsubsection{Photoacoustic imaging}

PAI is an imaging technique using pulsed laser irradiation to generate US waves which are subsequences of pressure changes due to thermal expansion and relaxation. The generated initial pressure is described as [23-25]:

$$
p=\Gamma \mu_{a} \Phi,
$$

where $\mu_{a}$ is the absorption coefficient $\left[\mathrm{cm}^{-1}\right], \Phi$ is the light fluence $\left[\mathrm{J} / \mathrm{cm}^{2}\right]$, and $\Gamma$ (Grüneisen parameter) is a dimensionless parameter and is defined as $\Gamma=\beta c^{2} / C_{P}$, where $\beta$ is the thermal expansion coefficient $\left[\mathrm{K}^{-1}\right], c$ is the speed of sound $[\mathrm{m} / \mathrm{s}]$, and $C_{P}$ is the isobaric specific heat $[\mathrm{J} / \mathrm{kgK}]$.

Light propagating in the tissue is scattered and absorbed. Since scattering and absorption are strongly dependent on the wavelength, light at different wavelengths reaches different depths $[26,27]$. Therefore, the light fluence inside the tissue depends on both the excitation wavelength and the position.

The absorption coefficient, $\mu_{a}$, is a wavelength-dependent optical property of the absorber. The generated initial pressure, $p$, can be rewritten as a function of the excitation wavelength and the local position:

$$
p=f(\lambda, x, y, z) .
$$

\subsubsection{Reflection artifacts in photoacoustic imaging}

Fig. 3.1 illustrates the principle of RAs in PAI. A part of generated US waves (blue) is reflected at the acoustic reflector, seen in Fig. 3.1(a) The reflected US waves (red) propagating back to the detector resemble a virtual acoustic source, so-called RA, located at a larger depth. Fig. 3.1(b) is a reconstructed PA image of a phantom representing this situation. The phantom was made of a black thread placed above a plastic petri dish lid, and demi-water was used as an acoustic coupling medium. An RA at a larger depth is clearly visible in this image. 

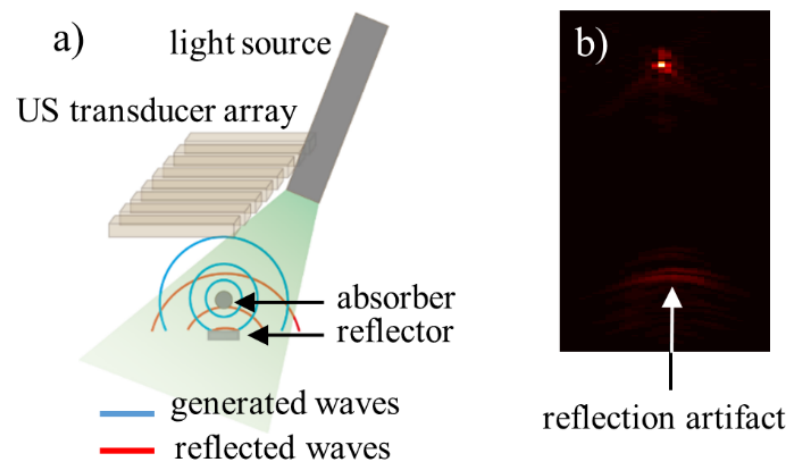

Fig. 3.1. RA in PAI. (a) A deep reflector leads to reflecting US waves. (b) An acquired PA image of a phantom (a black thread placed above a plastic petri dish lid) embedded in demi-water represents this situation.

In a clinical scenario where there are a few blood vessels located above a tumor. The tumor can reflect US signals generated from the blood vessels causing RAs surrounding the tumor. This can negatively affect the ability to assess tumors based on oxygen saturation of hemoglobin $[3,28]$.

\subsubsection{Method}

The principle of our method is based on Eq. (3.2) where the pixel value in the acquired PA image represents the generated initial pressure as a function of the local light fluence and the excitation wavelength. Exploiting this principle with multi-wavelength PAI, a sequence of PA images with multiple wavelengths of light is obtained. As all images are of the same region of interest (ROI), they show the same structure of the sample. Studying the changes of the pixel values reveals the spectral responses of absorbers in the images. Our method relies on the following two assumptions:

1. Absorbers with identical optical properties located at different positions give different spectral responses due to different local light fluence.

2. Both direct and reflected PA signals convey the optical properties of the source.

If the above two assumptions are fulfilled, the spectral response of RAs is identical to the spectral response of their source (real absorber) and two identical absorbers will not be misidentified as one reflection artifact of the other. The first assumption is discussed further at the end of this section. 
Fig. 3.2 shows the flowchart of the method. Images of the sample are acquired with multiple wavelengths. Of the acquired images, the image giving the strongest signal is selected for segmentation which detects image features. The features extracted from the segmentation step are applied to all the other images to obtain their spectral response. This information is then used in the RA correction step to identify and remove RAs. The corrected image which is a segmented image is further processed through the de-segmentation step to recover the shape of the remaining features giving the final corrected image.

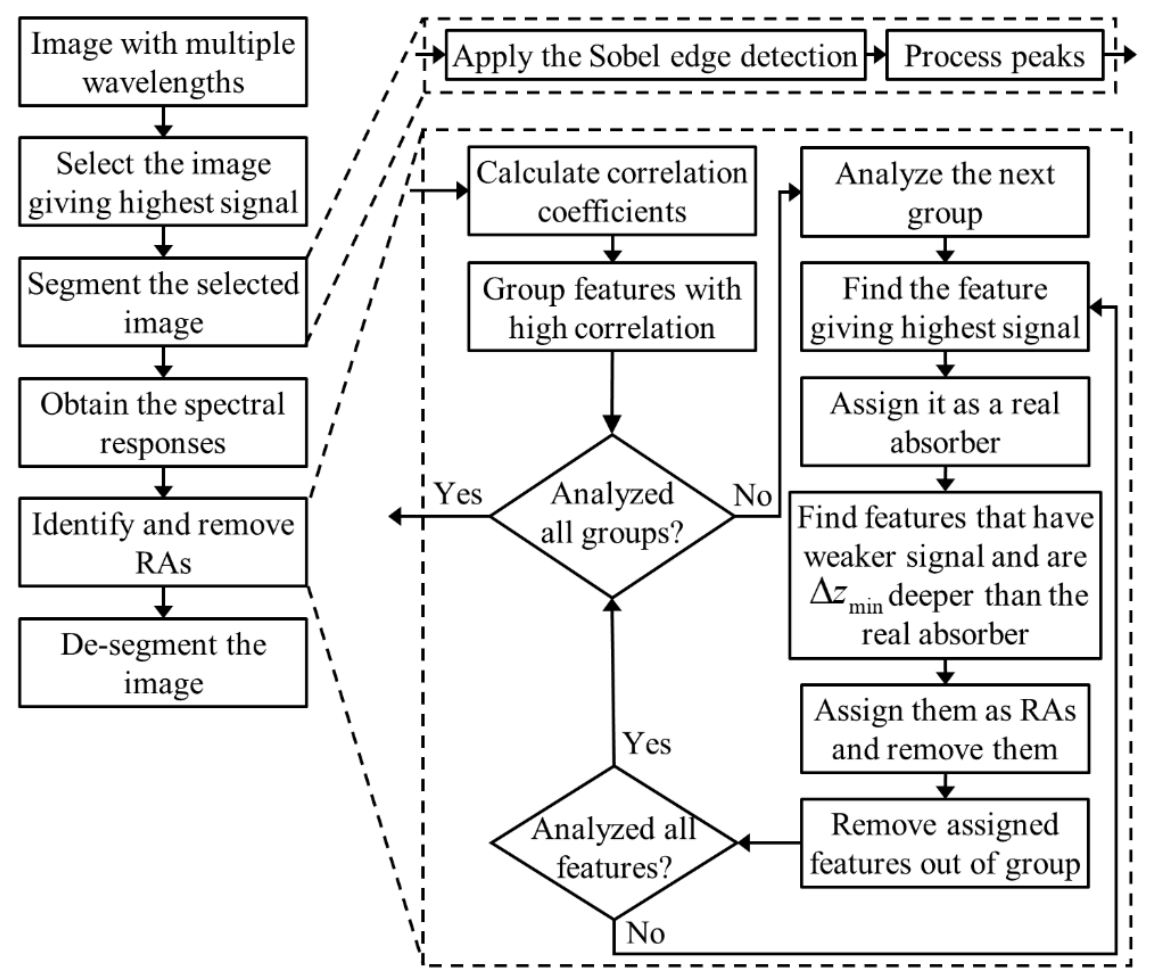

Fig. 3.2. The flowchart of the method.

In the segmentation step, an automatic segmentation algorithm which is based on the Sobel edge detection algorithm [29] is implemented to detect image features. Computing the Sobel edge threshold is supported by Matlab.

Fig. 3.3 illustrates this segmentation method. Fig. 3.3(a) is a sample PA image which represents two blood vessels (upward blue arrows) and their reflection (downward yellow arrows). Properly segmenting these features is expected. However, applying a threshold can 
lead to over-thresholding or under-thresholding. In the case of over-thresholding, weak edges cannot be detected resulting in feature loss. Fig. 3.3(b) shows an over-thresholding case where bottom features are not detectable. In contrast, Fig. 3.3(c) shows an underthresholding case. Several features are detected as one single feature in this case.

We avoid over-thresholding by choosing half of the threshold calculated by Matlab. As a consequence, under-thresholding might happen. We further process the image with a peakprocess. In this process, we find all peaks in the image and then set a part of pixels surrounding the peaks to zero. Under-thresholding is significantly improved after this step giving separate features, seen in Fig. 3.3(d). It might lead to over-segmentation in which one absorber is segmented into a number of features. However, since these features are parts of one absorber, they share the spectral response of the absorber.
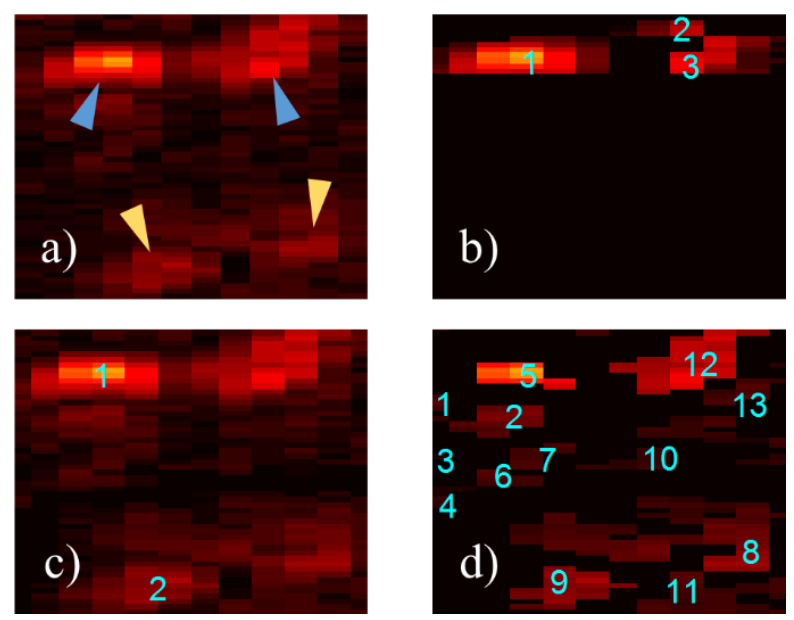

Fig. 3.3. An example of the segmentation process. (a) The original image showing two blood vessels (upward blue arrows) and their reflection (downward yellow arrows). (b) An over-thresholding segmented image. (c) An under-thresholding segmented image.

(d) The under-thresholding and peak-processed segmented image.

To obtain features spectral response, the detected features from the segmentation step are applied to all other images. Of each feature the maximum pixel value is taken from all images, giving that feature's spectral response. 
Spectral responses of all features are then compared to each other using the Pearson correlation coefficient [30]:

$$
\rho(A, B)=\frac{\operatorname{cov}(A, B)}{\sigma_{A} \sigma_{B}}
$$

where $\mathrm{A}$ and $\mathrm{B}$ are spectral responses of two features, $\operatorname{cov}(A, B)$ is the covariance of $\mathrm{A}$ and $\mathrm{B}, \sigma_{A}$ and $\sigma_{B}$ are the standard deviation of $\mathrm{A}$ and $\mathrm{B}$ respectively.

In the identifying and removing RAs step, a threshold, $\rho_{t h}$, is then applied to separate high correlation coefficients from all correlation coefficients. Features with spectral responses with correlations exceeding $\rho_{t h}$ are grouped together as suspicious RAs. In addition, RAs appear at a larger depth and have a weaker signal than the corresponding real absorber due to longer propagation and attenuation. An extra condition is used to identify RAs, that RAs must be deeper than their real absorber at least $\Delta z_{\min }$, which is described at the end of this section. Features in the each group are analyzed based on these conditions to identify RAs and thus remove them.

RAs are removed by setting the pixel values of the RA features to zero. Fig. 3.4(a) is the corrected image of Fig. 3.3(a). Features 8, 9, and 11 detected in Fig. 3.3(d) are removed. However, this corrected image is a segmented image. To recover the shape of the remaining features, the de-segmentation step is applied. All pixels surrounding the remaining peaks which were removed in the peak-process are recovered giving the final corrected image, seen in Fig. 3.4(b).
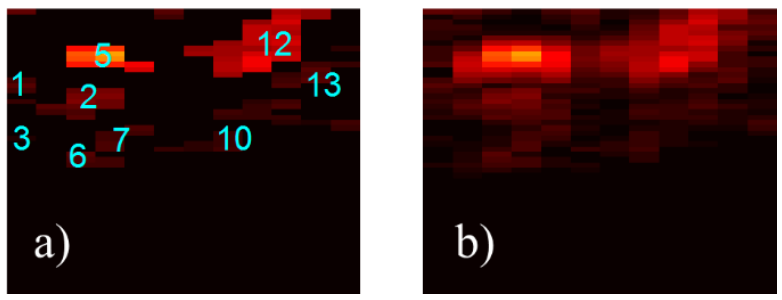

Fig. 3.4. An example of correcting RAs in PA images. (a) The corrected image of Fig.

3.3(a). (b) The final corrected image.

Segmentation benefits image feature analysis, however, a properly segmented image might be not obtained. Therefore, another approach without segmentation is also 
implemented for analyzing images. In this approach, each pixel of the image is considered as an object to study the spectral responses. Particularly, spectral responses of all pixels, rather than features, are correlated to each other. The analysis based on the correlation coefficient is the same as the analysis used for segmented features.

A comparison of the method with and without segmentation will be presented in the experimental results section, and further discussed in the discussion section.

In a highly scattering medium, a local region can have light fluence nearly homogeneous, thus two identical absorbers in that region might have the same spectral responses, giving a correlation coefficient exceeding $\rho_{t h}$. As a consequence, assumption 1 is not appropriate. To avoid this, a minimum distance $\Delta z_{\min }$ in the depth between the two features is used as an extra condition to assess that whether one feature is an RA of the other one or two separate absorbers with the same spectral response. The value of $\Delta z_{\text {min }}$ is related to the value of $\rho_{t h}$. In other words, $\Delta z_{\min }$ defines a region below a PA image feature where no other image features are assessed as its RAs. In addition, if one absorber is oversegmented, one segment would be considered as an RA. This can be avoided with $\Delta z_{\text {min }}$. To determine this $\Delta z_{\min }$, measurements were performed and will be reported in the experimental results section, and discussed further in the discussion section.

\subsection{Setup}

Experiments were carried out using a handheld PAI probe, depicted in Fig. 3.5. The handheld probe is connected to a commercial ultrasound scanner MyLabOne (Esaote Europe BV, The Netherlands) for the acquisition of US and PA images. The scanner can acquire data at a maximum sampling frequency of $50 \mathrm{MHz}$ with 12 bit digitization. This device was used in research mode so that raw data could be acquired in an external PC for offline processing.

The US transducer array in the handheld probe has a center frequency of $7.5 \mathrm{MHz}$ with a bandwidth of $100 \%$. It comprises 128 elements with a pitch of $0.3 \mathrm{~mm}$. For our study the central 64 elements were used. Diode lasers integrated into the handheld probe emit light at 4 different wavelengths $(808,915,940$, and $980 \mathrm{~nm})$ at a repetition rate of up to $10 \mathrm{kHz}$. 


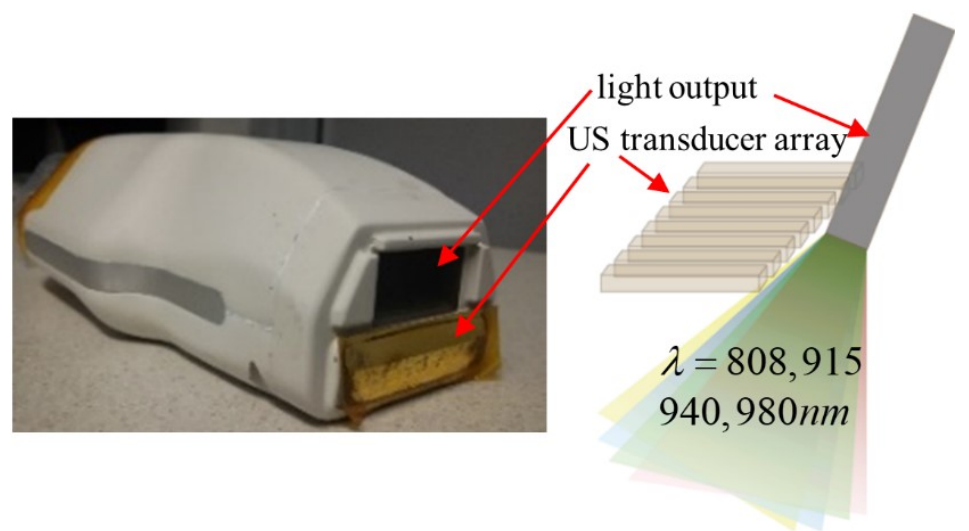

Fig. 3.5. Photo and schematic drawing of the handheld probe.

Table 3.1 presents specifications of the diode lasers working at the repetition rate of 1 $\mathrm{kHz}$. In addition, the angles at the output are 53.1, 55.6, 47.8, and 50.3 degrees at 808,915 , 940 , and $980 \mathrm{~nm}$, respectively, due to diode lasers placed at different stacks and a prism at the light output. These differences between wavelengths add to the light fluence variation of these wavelengths.

Table 3.1. Lasers specifications at a repetition rate of $1 \mathrm{kHz}$.

\begin{tabular}{llll}
\hline wavelength $[\mathrm{nm}]$ & $\begin{array}{l}\text { pulse energy } \\
\text { at the output }[\mathrm{mJ}]\end{array}$ & pulse width $[\mathrm{ns}]$ & $\begin{array}{l}\text { Fluence at the } \\
\text { output }\left[\mathrm{mJ} / \mathrm{cm}^{2}\right]\end{array}$ \\
\hline 808 & 0.96 & 84.2 & 1.04 \\
915 & 0.98 & 88 & 1.01 \\
940 & 0.89 & 98.9 & 0.95 \\
980 & 0.82 & 94.2 & 0.87
\end{tabular}

Offline processing of data was done on a PC (Intel Core i7 $3.41 \mathrm{GHz}, 8 \mathrm{~GB}$ of RAM) running Matlab R2016b.

\subsection{Experimental results}

To demonstrate the feasibility of the method, we performed experiments in phantoms as well as in vivo. The PA image reconstruction was done using a Fourier transform based reconstruction algorithm [31].

In each experiment, 4 laser pulses of 4 different wavelengths followed by 1 US pulse were sent repeatedly for 100 times. 4 PA images at 4 different wavelengths and 1 US image 
were then acquired by averaging signal over 100 pulses. The diode lasers were run at a repetition rate of $1 \mathrm{kHz}$. The US image was used to verify the location of absorbers and corresponding RAs.

\subsubsection{Phantoms}

A phantom was made of two black threads, with the diameter of 200-250 $\mu \mathrm{m}$, and a petri dish lid (Greiner Bio-One GmbH, Germany) as an acoustic reflector, with thickness of $750 \mu \mathrm{m}$, seen in Fig. 3.6(a). A schematic drawing of a cross-section of the phantom is shown in Fig. 3.6(b). The lid was positioned underneath one black thread as an acoustic reflector. The phantom was mounted on a mount (CP02/M, Thorlabs, Germany) to fixate it in a solution of $3.5 \%$ Intralipid 20\% (Fresenius Kabi, The Netherlands) in demi-water. This solution served as an acoustic coupling medium as well as an optically scattering background. The reduced scattering coefficient of the solution was estimated as $\mu_{s}^{\prime}=6 \mathrm{~cm}^{-1}$ at the wavelength of $900 \mathrm{~nm}$ based on [32]. Fig. 3.6(c) shows a combined PA and US image illustrating a cross-section of this phantom. The gray color part is the US image showing two surfaces of the lid which reflect US waves. The hot color part is the PA image where two black threads are visualized at expected positions relative to the lid. Underneath the lid were some more features. As there was no absorbers underneath the lid, these features were RAs of the black thread above the lid. In the PA image, there is a long "tail" of the absorber above the lid which perhaps is a reconstruction artifact.

4 PA images of the ROI corresponding to 4 wavelengths are shown in Fig. 3.6(d). The intensity of the reflections was not strong. The explanation might be that the acoustic reflectivity of the petri dish lid in that coupling medium was not high. On the other hand, in the US image's case, the US transducer array generated higher pressure compared to PA signal resulting in high intensity of the reflector in the acquired US image. The image acquired at a $940 \mathrm{~nm}$ wavelength had the strongest signal therefore this image was selected for segmentation. 


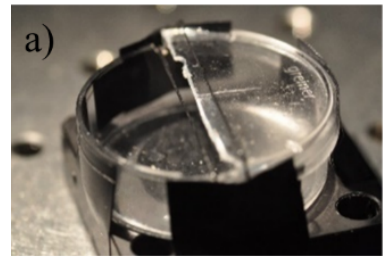

b)
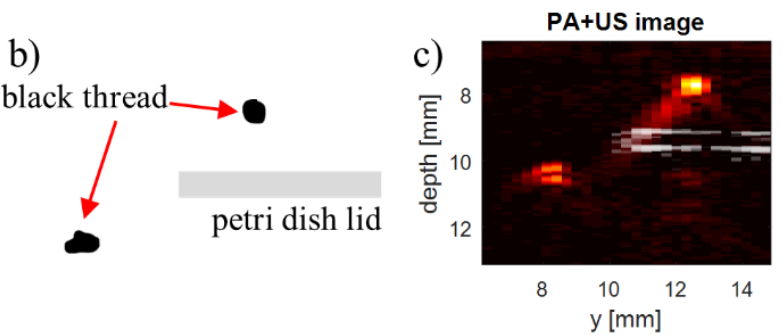

d)
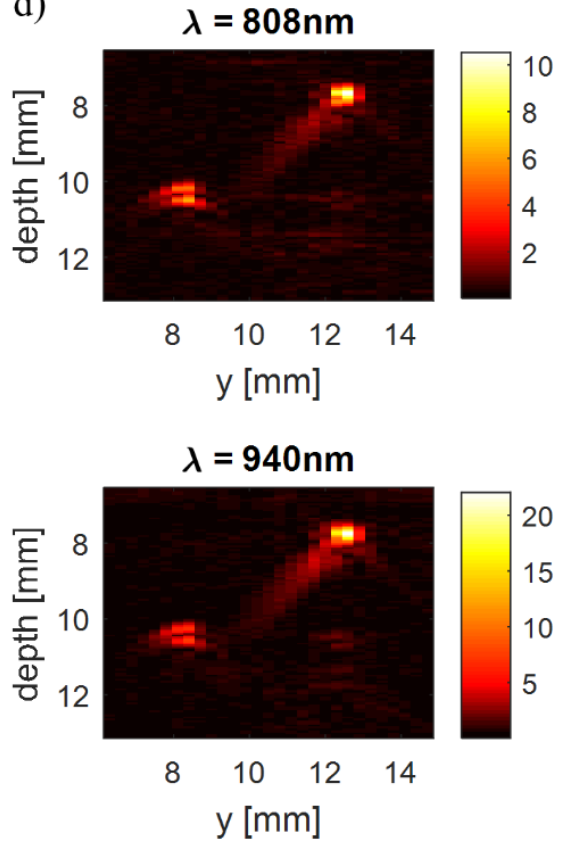
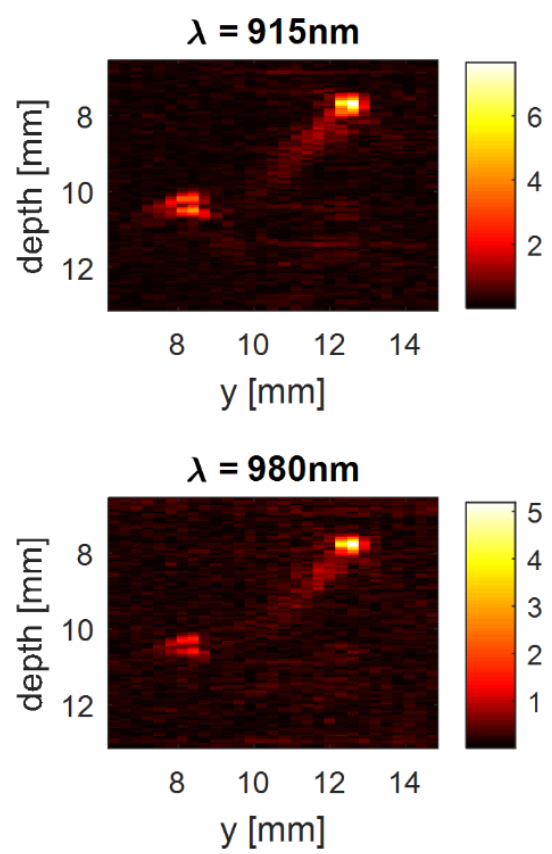

Fig. 3.6. (a) A phantom used for experiments. (b) A schematic cross-section of the phantom (c) Combined PA and US image. (d) 4 PA images acquired at 4 wavelengths $(808,915,940$, and $980 \mathrm{~nm})$.

Fig. 3.7(a) shows the segmented image acquired at 940nm with numbered features (see also Appendix 1.1). The spectral response of several features is shown in Fig. 3.7(b) (the spectral response was normalized with the maximum value). 

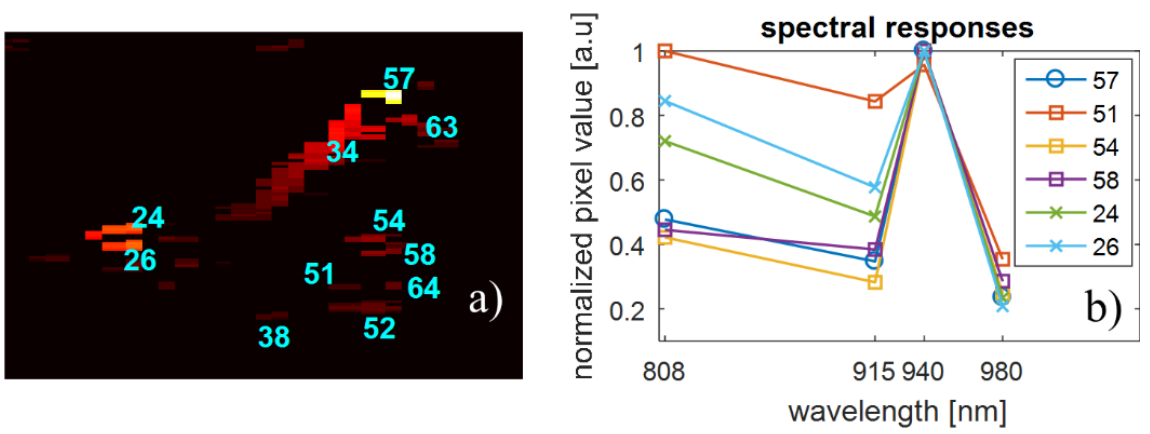

Fig. 3.7. Image analysis of a phantom experiment. (a) Segmented image with numbered features, and all pixels in a feature being assigned the maximum value of that feature. (b) Maximum normalized spectral responses of the features.

Two black threads made of the same materials have identical optical absorption properties. However, from Fig. 3.7(b), the difference in the spectral response of these two absorbers is observable (feature 57 for one thread and features 24 and 26 for another thread). This matches with the first assumption described in the method section. On the other hand, spectral responses of feature 57 and its RAs, features 54 and 58, are highly identical. In other words, optical properties of the absorber were conserved in the US waves in spite of reflection confirming the second assumption.

Of the obtained spectral responses mutual correlations are calculated to determine "similarity" of the responses. Table 3.2 shows the correlation coefficients of these responses. The correlation coefficients between feature 57 and features 52, 54, 58, and 64 are high (close to 1) and these features appear at larger depths than feature 57 with a lower signal revealing that they are RAs of feature 57. The correlation coefficients of feature 57 and features 24 and 26 are 0.937 and 0.848 respectively. A threshold $\rho_{t h}$ in between 0.94 and 0.97 would be sufficient to separate features 24 and 26 from features 52, 54, 58 and 64 . Feature 51, which is likely an RA of feature 57, however has a low correlation coefficient. This can be explained as the intensity of feature 51 is close to the background, that would highly affect the spectral response and thus the correlation. 
Table 3.2. Correlation coefficients of obtained spectral responses in the phantom experiment (see also Appendix 2.1).

\begin{tabular}{lllllllll}
\hline Feature & 24 & 26 & 51 & 52 & 54 & 57 & 58 & 64 \\
\hline 24 & 1 & 0.979 & 0.843 & 0.974 & 0.898 & 0.937 & 0.901 & 0.928 \\
26 & 0.979 & 1 & 0.932 & 0.912 & 0.791 & 0.848 & 0.798 & 0.846 \\
51 & 0.843 & 0.932 & 1 & 0.702 & 0.532 & 0.618 & 0.557 & 0.643 \\
52 & 0.974 & 0.912 & 0.702 & 1 & 0.963 & 0.978 & 0.952 & 0.951 \\
54 & 0.898 & 0.791 & 0.532 & 0.963 & 1 & 0.994 & 0.995 & 0.977 \\
57 & 0.937 & 0.848 & 0.618 & 0.978 & 0.994 & 1 & 0.995 & 0.99 \\
58 & 0.901 & 0.798 & 0.557 & 0.952 & 0.995 & 0.995 & 1 & 0.992 \\
64 & 0.928 & 0.846 & 0.643 & 0.951 & 0.977 & 0.99 & 0.992 & 1
\end{tabular}

In this experiment, $\rho_{t h}=0.95$ was applied identifying features 52, 54, 58 and 64 as RAs. These features were subsequently removed from the image giving a corrected segmented image, Fig. 3.8(a). This corrected image was then de-segmented to obtain the final corrected image, seen in Fig. 3.8(b). Feature 51 was not removed. However, as this feature is close to the noise level, it does not considerably affect the interpretation of the image. Fig. 3.8(c) shows a comparison of the acquired PA image and the final corrected image.
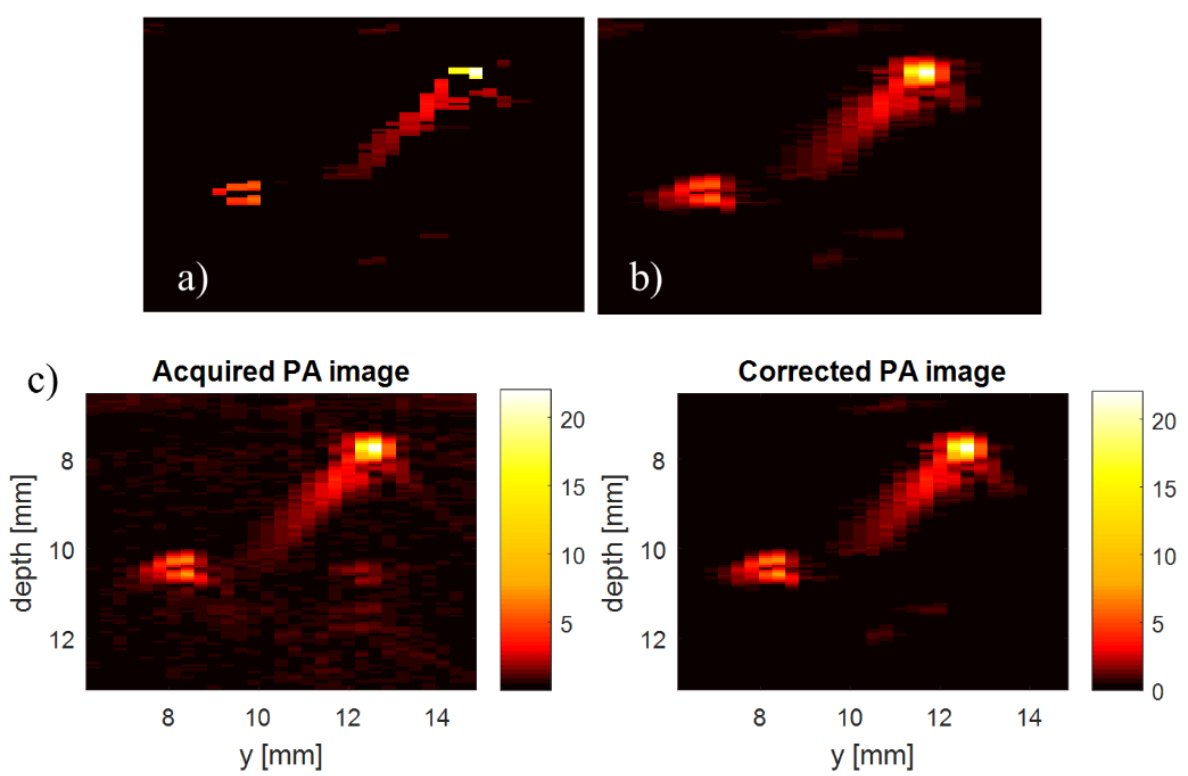

Fig. 3.8. Processing features in an acquired PA image of the phantom. (a) The corrected segmented image. (b) The final corrected image after de-segmentation. (c) A comparison of the acquired PA image and the final corrected image. 


\subsubsection{In vivo}

We also assessed the method with in vivo experiments. Our experiments focused on fingers where bones are close underneath the skin giving clear RAs. Fingers were placed $7 \mathrm{~mm}$ underneath the probe and demi-water was used as the US coupling medium.

Fig. 3.9(a) shows an in vivo PA image of a cross-section of a healthy volunteer's finger. Fig. 3.9(d) is a photo depicting the experimental configuration. Acquired PA and US images are represented in Fig. 3.9(a) and (b) respectively. The US image is compared with Fig. 3.9(c), adapted from [33], revealing the periosteum and bone. In the PA image, features beneath the periosteum and bone are therefore disclosed as RAs of the skin and blood vessels.

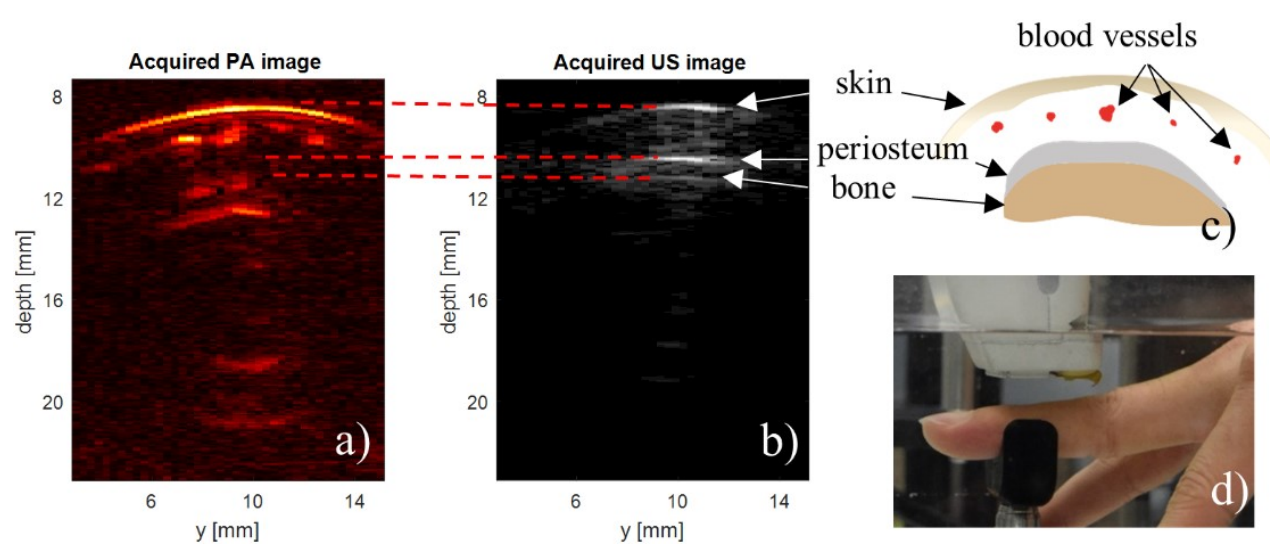

Fig. 3.9. RAs in in vivo PAI. Acquired PA (a) and US (b) images of a finger. (c) An image adapted from "Sobotta: Atlas of human anatomy" [33] shows a cross-section of a finger. (d) A photo of an in vivo imaging experiment.

The finger was imaged with 4 wavelengths and the acquired images were processed and analyzed the same as described in the phantoms section. The processed image with numbered features (see also Appendix 1.2) and several spectral responses are shown in Fig. 3.10. Features 56 and 63, 77 and 62, 32 and 50 have similar spectral responses (the correlation coefficient of these pairs is higher than 0.99), validating the second assumption. 

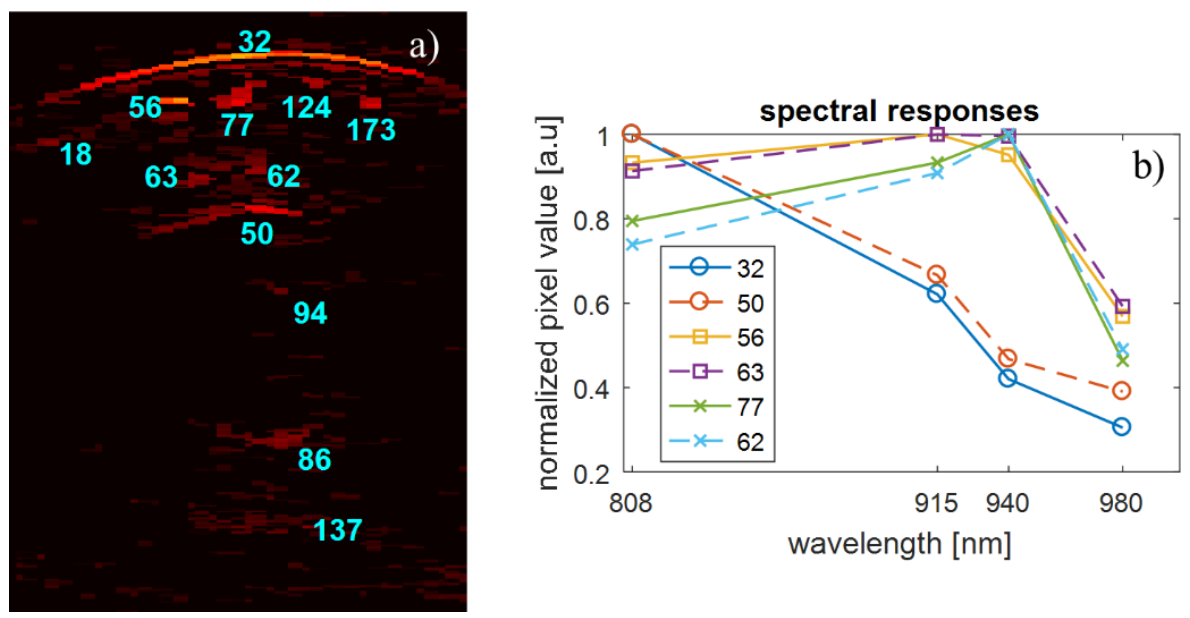

Fig. 3.10. Image analysis of an in vivo experiment. (a) A segmented image with numbered features. (b) Spectral responses of the features.

The spectral responses of features 32 and 56 are observably different from each other as they represent different chromophores (melanin and blood). Interestingly, features 56 and 77 representing two blood vessels also have different spectral responses. This might be a result of different $\mathrm{sO}_{2}$ in these blood vessels, or of different local spectra of the excitation light.

It is worth noting that feature 50 which is an RA of the skin has higher intensity than features 18 and 124 which are two blood vessels. Increasing the segmentation threshold to remove feature 50 will also remove features 18 and 124 leading to losing real features.

Fig. 3.11 shows acquired and corrected images where all RAs were identified and removed. 

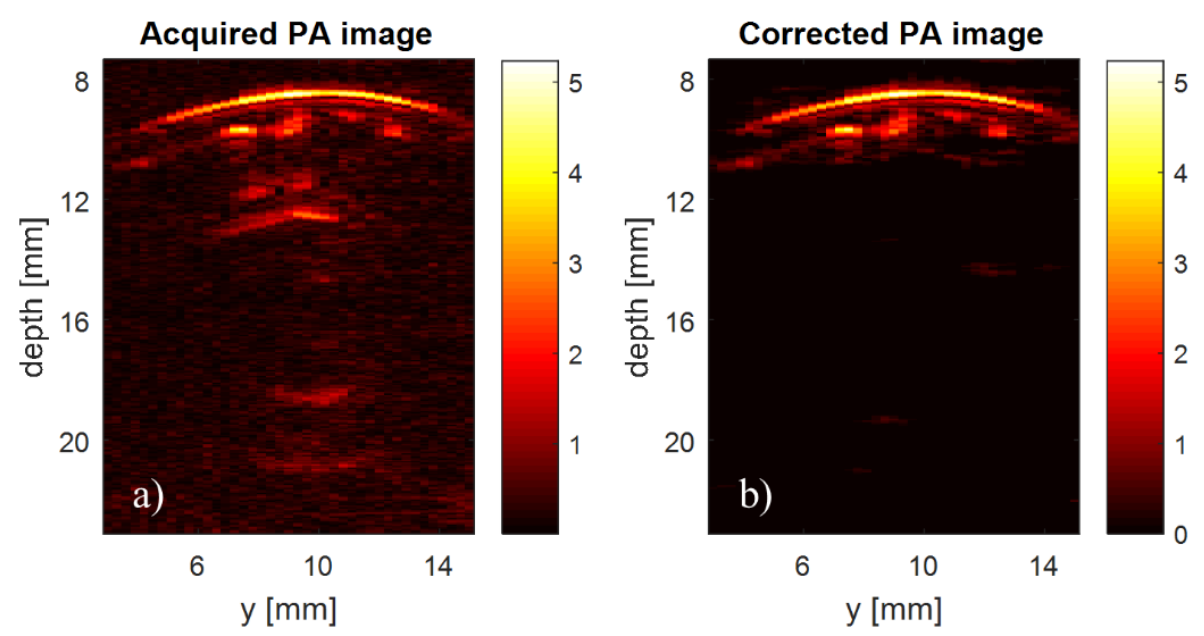

Fig. 3.11. Correcting RAs in an in vivo imaging experiment. (a) An acquired PA image of a finger. (b) The corrected image.

$\rho_{t h}=0.95$ was used in this experiment (see Appendix 2.2 for a complete correlation coefficient table).

\subsubsection{Minimum vertical distance $\Delta z_{\min }$}

As mentioned in the method section, in a region having nearly homogeneous light fluence, two identical absorbers with a vertical distance (along depth) less than $\Delta z_{\min }$ might have the same spectral responses, giving a correlation exceeding $\rho_{t h}$, which results in failure of assumption 1 formulated in section Method. The method, therefore, might misidentify and miscorrect one absorber as an RA of the other one.

To evaluate the correlation as a function of $\Delta z$, we performed several experiments comparing the spectral responses of two identical absorbers in a medium mimicking tissue optical properties. The medium was a solution of $2 \%$ Intralipid $20 \%$ with estimated $\mu_{s}^{\prime}=3.5$ $\mathrm{cm}^{-1}$ at the wavelength of $900 \mathrm{~nm}$ [32], $10^{-4}$ volume fraction of India ink (Royal Talens, The Netherlands), and $4 \times 10^{-4}$ volume fraction of black ecoline (Royal Talens, The Netherlands) in Milli-Q water. The absorption coefficient of this solution (without Intralipid) was 0.599, $0.456,0.534$, and $0.776 \mathrm{~cm}^{-1}$ at the wavelength of $808,915,940$, and $980 \mathrm{~nm}$ respectively, measured using a photo-spectrometer (UV-2600, Shimadzu, The Netherlands). Two black suture wires (USP 3/0, diameter of $0.24 \mathrm{~mm}$, Vetsuture Nylon, The Netherlands) mimicking 
two identical absorbers were used. These two wires were embedded in the solution, one was fixated and the other one was attached to a motorized translation stage (MTS50A-Z8, Thorlabs, Germany) to adjust the vertical distance $\Delta z$ between the two wires.

At each $\Delta z$, PA images were acquired using the four wavelengths. Images were then processed to calculate the correlation coefficient of spectral responses of the two wires. The measurement was repeated 4 times at each distance $\Delta z$. The average, maximum and minimum values are shown in Fig. 3.12(a) presenting the behavior of the correlation coefficient with the distance. Fig. 3.12(b) shows spectral responses of the two wires of a measurement at $\Delta z_{1}=0.68 \mathrm{~mm}$ and a measurement at $\Delta z_{2}=2.1 \mathrm{~mm}$. The correlation coefficients at $\Delta z_{1}$ and $\Delta z_{2}$ in these measurements were 0.9916 and 0.9126 respectively.
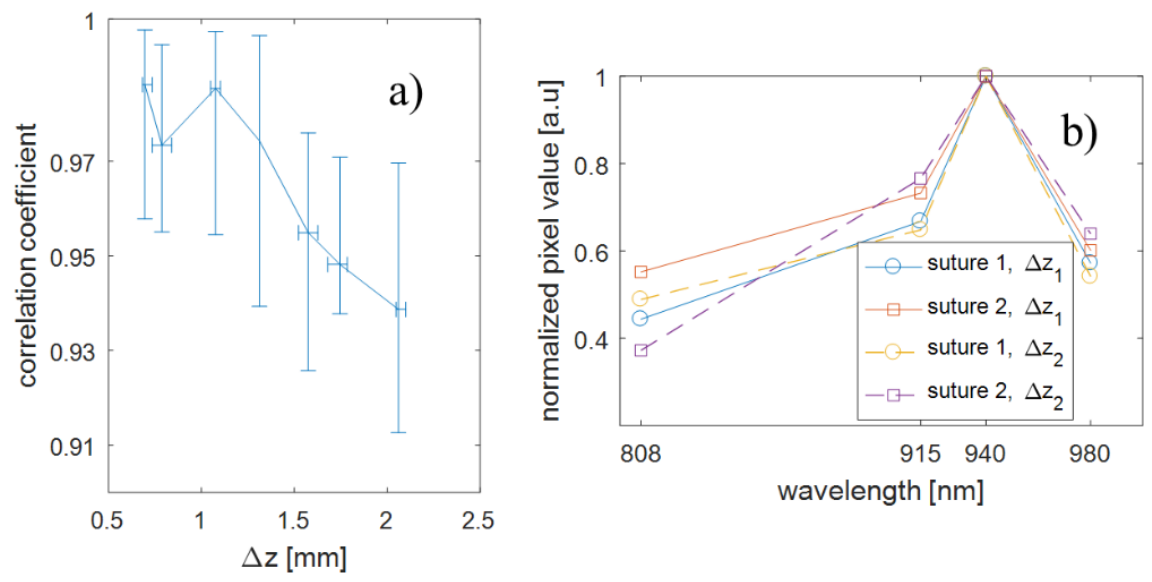

Fig. 3.12. (a) Correlation coefficient of spectral responses of two identical absorbers versus their vertical distance. (b) Spectral responses of the two suture wires at two different distances $\Delta z_{1}=0.68 \mathrm{~mm}$ and $\Delta z_{2}=2.1 \mathrm{~mm}$.

For a threshold of the correlation coefficient of $0.95, \Delta z_{\min }$ is $1.7 \mathrm{~mm}$. The vertical error bar in Fig. 3.12(a) is large. The reason for this might be that the correlation coefficient of spectral responses was calculated with only 4 wavelengths. For the Pearson correlation coefficient, 3 data points can give a meaningful coefficient. However, a small number of data points would affect the confidence interval [34]. 


\subsubsection{Comparison of the method with and without segmentation}

Two approaches for analyzing acquired images were mentioned in the method section, with and without segmentation.

For the method without segmentation, the spectral response of each pixel of the image is compared to all other pixels. Fig. 3.13(a) shows again the in vivo image presented in the in vivo section. Fig. 3.13(b) illustrates a pixel (the top red pixel) in the skin. The spectral response of this pixel is compared to all other pixels at least $2 \mathrm{~mm}$ below it, giving the correlation coefficient map. Red color indicates the correlation coefficient above 0.95 . Fig. 3.13(c) depicts identified RAs (yellow pixels, except the considered pixel) of the PA signal in the considered pixel.
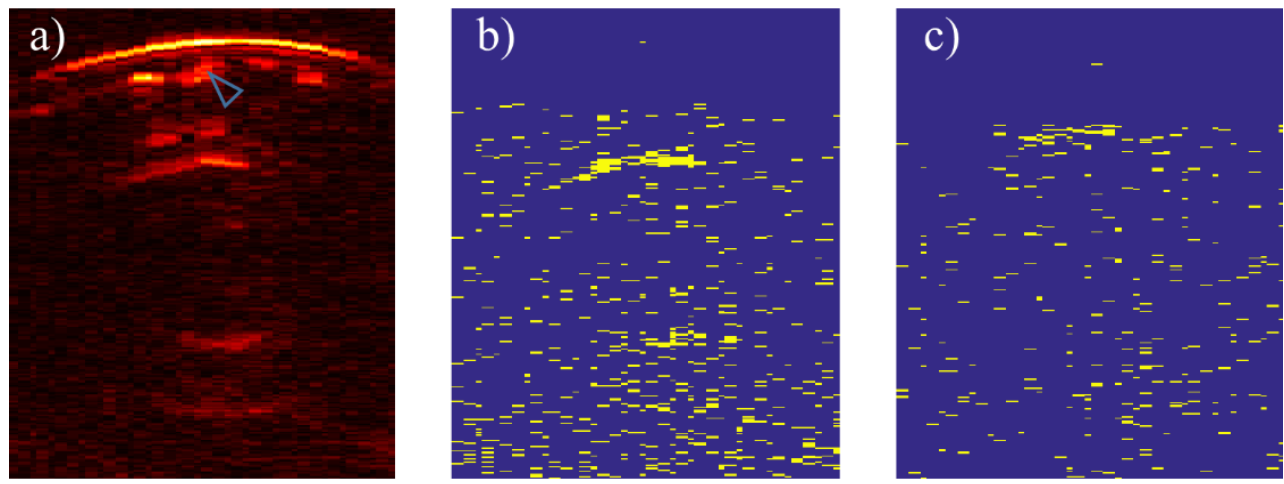

Fig. 3.13. RA identification of the method without segmentation in an in vivo image.

(a) An in vivo PA image. (b) The correlation coefficient map of a pixel in the skin with others at least $2 \mathrm{~mm}$ below the considered pixel (values above 0.95 are colored red).

(c) Identified RAs (yellow pixels) of the considered pixel.

Fig. 3.14 shows the results using the two approaches. The top images are an acquired PA image, the corrected image with segmentation, and the corrected image without segmentation of the phantom. The bottom images are images in vivo in the same order. 

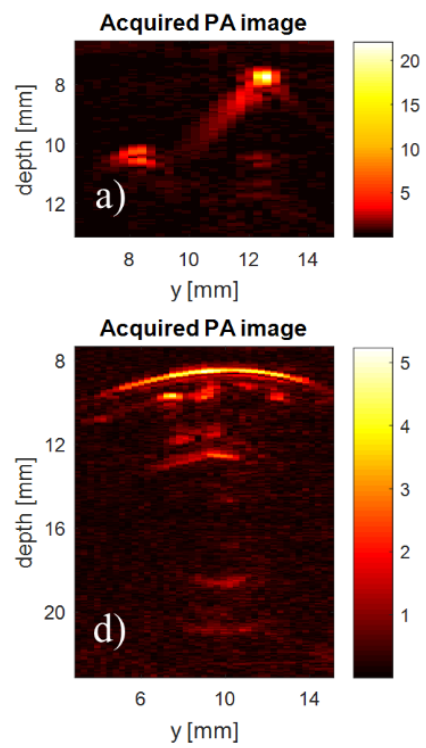

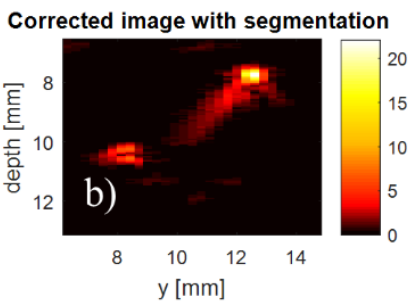

Corrected image with segmentation

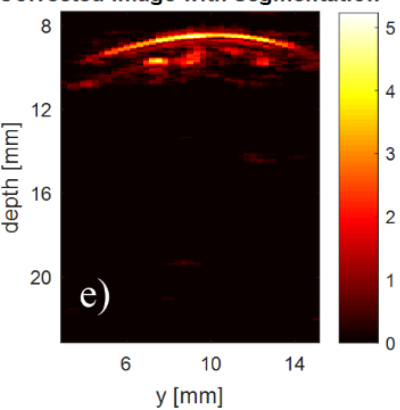

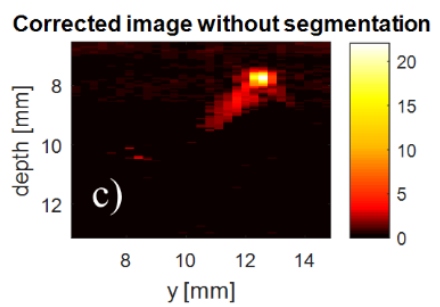

Corrected image without segmentation

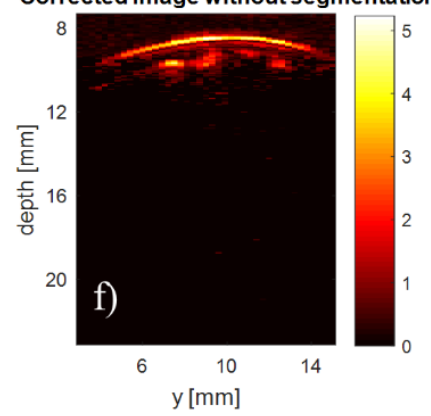

Fig. 3.14. Comparison of the method with and without segmentation. (a), (b), (c) An acquired PA image, the corrected image with segmentation, and the corrected image without segmentation of the phantom, respectively. (d), (e), and (f) An acquired PA image, the corrected image with segmentation, and the corrected image without segmentation in vivo, respectively.

In the corrected images without segmentation, the absorber in the bottom left corner of the phantom was observably shrunk. This could be due to the small amount of data points for the Pearson correlation coefficient.

The "tail" of the absorber in the top right corner of the phantom is likely a reconstruction artifact. A part of it was removed in the corrected image without segmentation. The reason is that the removed pixels in the "tail" were identified as RAs of the absorber.

The threshold for the method without segmentation was applied the same as the threshold for the method with segmentation (0.95). However, $\Delta z_{\min }$ was set as $2 \mathrm{~mm}$ for compensating the size of features.

\subsection{Discussion}

In in vivo imaging, the RAs of the blood vessels look like excess vessels and could be erroneously recognized as angiogenesis or hyper-vascularization, hallmarks of various diseases such as cancer or rheumatoid arthritis. With not much experience of RAs, 
misdiagnosis might happen. Simple solutions such as thresholding or limiting the imaging depth may be able to remove RAs in cases that the RAs are not accompanied by real image features with the same amplitude or depth range. If such image features exist, however, these simple solutions are not appropriate.

Compared to previously reported methods for reducing RAs, the proposed method offers significant advantages. First of all, the method works automatically and performing it does not require experience or training of the users, as is the case with DCA or PAFUSion in which the users have to hold the probe perpendicularly to the acoustic reflectors. Secondly, no US image is needed. Acquiring US images with multiple plane wave angles is more time consuming and comes at a higher processing expense. Thirdly, as the method does not need US images to detect RAs, matching features between PA and US images (as in PAFUSion) is not necessary resulting in detected RAs being completely removed in this proposed method. Fourthly, unlike deep learning, the method does not require training with various generated PA distribution sizes and geometries, and acoustic characteristic of the sample which might be unknown in in vivo imaging. Finally, the proposed method enhances the advantages of multispectral PAI.

Out-of-plane artifacts (direct and indirect out-plane-artifacts) can appear in the acquired PA images, especially artifacts caused by the skin. PAFUSion does not work for indirect out-of-plane artifacts. In contrast, they can be treated under the proposed method if the direct out-of-plane artifacts also appear in the image. However, both methods cannot handle direct out-of-plane artifacts. Another method for these artifacts is needed. Our future work will focus on a complete method combining this proposed method and a new method for out-ofplane artifacts.

The proposed method exploits the variance of light distribution of different wavelengths. In other words, the local illumination spectrum needs to be different at different depths for the method to work. In a scenario that the absorption and scattering spectrum is flat, resulting in a similar illumination spectrum at different depths, this method will not work. However, this is not likely in clinical imaging where the absorption coefficient varies with wavelength and various tissues [26]. 
$\Delta z_{\text {min }}$, which defines a region below an original PA feature where the method will not correct its RAs, is the main limitation of this method. In our experiments demonstrating the method, $\Delta z_{\min }$ was $1.7 \mathrm{~mm}$ corresponding to the threshold of the correlation coefficient of 0.95. However, this value is not a critical limitation in clinically relevant scenarios. In in vivo imaging, with different layers of tissue such as skin and muscle, optical heterogeneity would be stronger resulting in a smaller $\Delta z_{\min }$ than in this study. Additionally, $\Delta z_{\min }$ can be further reduced by selecting wavelengths at which light fluence varies strongly along the depth.

The proposed method uses PA information from the acquired PA images with multiple wavelengths. In principle, one PA image can be reconstructed from one laser pulse per excitation wavelength, therefore the variability in laser pulses is not a limiting factor for the principle of the proposed mothed. In our experiments, to improve the signal to noise ratio, we performed averaging over 100 laser pulses per excitation wavelength. In this case, pulse to pulse variation in beam shape and pointing stability might impede the performance of our method. However, our results show that this is not the case in our probe.

In this method, pixel values in the acquired image are considered to represent the generated initial pressure. However, the reconstruction algorithm can produce image artifacts itself and thus affect the method. We did not observe strong effect of reconstruction artifacts in our work. Nevertheless, an appropriate reconstruction algorithm should be considered.

The method might not be able to identify an RA if it appears at the same position with another absorber or another RA. In that case, they appear as one single feature and therefore, the spectral response of this feature will not highly correlate with the RA's real absorber. Another approach such as PAFUSion is needed to identify the RAs.

A small number of data points might affect the Pearson correlation coefficient [34]. The relation between the number of wavelengths and the efficacy of our method will be investigated in the future work.

Our method is a post-processing method. Therefore, compared to pure PAI, it costs more calculation and thus reduces the frame rate. However, for images with the size of $512 \times 64$ pixels ( $\sim 25 \mathrm{~mm}$ depth), correcting with and without segmentation take $\sim 0.2$ seconds and $\sim$ 
2-3 seconds running in Matlab, respectively, showing the potential of the method running in real-time. For the method without segmentation, if the memory of the computing device is sufficient ( 12 GB for this case) vectorizing data can be accomplished reducing time consumption significantly. Since the imaging depth capability of this handheld probe is $\sim 10$ $\mathrm{mm}$, the size of images can be reduced resulting in less expensive calculation. Nevertheless, a GPU can be utilized for further acceleration of the method, especially without segmentation.

\subsection{Conclusion}

The proposed method can identify and remove in plane reflection artifacts (RAs) in PAI exploiting local light fluence variation between multiple wavelengths. Experiments in phantoms and in vivo were performed for a proof of concept, using 4 wavelengths: 808, 915, 940 and $980 \mathrm{~nm}$. Results show the potential of the method for correcting RAs in real-time with no separate ultrasound images needed. In addition, experiments were carried out using a compact PAI system suitable for clinical use, demonstrating the practical applicability of this method for medical use. 


\subsection{References}

[1] P. K. Upputuri, and M. Pramanik, "Recent advances toward preclinical and clinical translation of photoacoustic tomography: a review," Journal of Biomedical Optics 22(4), 041006 (2016).

[2] M. Heijblom, D. Piras, F. M. van den Engh, M. van der Schaaf, J. M. Klaase, W. Steenbergen, and S. Manohar, "The state of the art in breast imaging using the Twente Photoacoustic Mammoscope: results from 31 measurements on malignancies," European radiology 26(11), 3874-3887 (2016).

[3] M. Toi, Y. Asao, Y. Matsumoto, H. Sekiguchi, A. Yoshikawa, M. Takada, M. Kataoka, T. Endo, N. Kawaguchi-Sakita, and M. Kawashima, "Visualization of tumor-related blood vessels in human breast by photoacoustic imaging system with a hemispherical detector array," Scientific Reports 7, (2017).

[4] P. J. van den Berg, K. Daoudi, H. J. B. Moens, and W. Steenbergen, "Feasibility of photoacoustic/ultrasound imaging of synovitis in finger joints using a point-of-care system," Photoacoustics 8, 8-14 (2017).

[5] J. Jo, G. Xu, M. Cao, A. Marquardt, S. Francis, G. Gandikota, and X. Wang, "A Functional Study of Human Inflammatory Arthritis Using Photoacoustic Imaging," Scientific reports 7(1), 15026 (2017).

[6] K. Jansen, M. Wu, A. F. van der Steen, and G. van Soest, "Photoacoustic imaging of human coronary atherosclerosis in two spectral bands," Photoacoustics 2(1), 12-20 (2014).

[7] K. Daoudi, P. Van Den Berg, O. Rabot, A. Kohl, S. Tisserand, P. Brands, and W. Steenbergen, "Handheld probe integrating laser diode and ultrasound transducer array for ultrasound/photoacoustic dual modality imaging," Optics express 22(21), 2636526374 (2014).

[8] J. J. Niederhauser, M. Jaeger, R. Lemor, P. Weber, and M. Frenz, "Combined ultrasound and optoacoustic system for real-time high-contrast vascular imaging in vivo," IEEE transactions on medical imaging 24(4), 436-440 (2005). 
[9] C. Kim, T. N. Erpelding, L. Jankovic, M. D. Pashley, and L. V. Wang, "Deeply penetrating in vivo photoacoustic imaging using a clinical ultrasound array system," Biomedical optics express 1(1), 278-284 (2010).

[10] C. Haisch, K. Eilert-Zell, M. M. Vogel, P. Menzenbach, and R. Niessner, "Combined optoacoustic/ultrasound system for tomographic absorption measurements: possibilities and limitations," Analytical and bioanalytical chemistry 397(4), 15031510 (2010).

[11] K. Sivasubramanian, V. Periyasamy, R. A. Dienzo, and M. Pramanik, "Hand-held, clinical dual mode ultrasound-photoacoustic imaging of rat urinary bladder and its applications," Journal of biophotonics 11(5), e201700317 (2018).

[12] K. Sivasubramanian, V. Periyasamy, and M. Pramanik, "Non-invasive sentinel lymph node mapping and needle guidance using clinical handheld photoacoustic imaging system in small animal," Journal of biophotonics 11(1), e201700061 (2018).

[13] M. K. A. Singh, V. Parameshwarappa, E. Hendriksen, W. Steenbergen, and S. Manohar, "Photoacoustic-guided focused ultrasound for accurate visualization of brachytherapy seeds with the photoacoustic needle," Journal of biomedical optics 21(12), 120501 (2016).

[14] M. Jaeger, L. Siegenthaler, M. Kitz, and M. Frenz, "Reduction of background in optoacoustic image sequences obtained under tissue deformation," Journal of biomedical optics 14(5), 054011 (2009).

[15] M. Jaeger, J. C. Bamber, and M. Frenz, "Clutter elimination for deep clinical optoacoustic imaging using localised vibration tagging (LOVIT)," Photoacoustics 1(2), 19-29 (2013).

[16] H.-M. Schwab, M. F. Beckmann, and G. Schmitz, "Photoacoustic clutter reduction by inversion of a linear scatter model using plane wave ultrasound measurements," Biomedical Optics Express 7(4), 1468-1478 (2016).

[17] M. K. A. Singh, and W. Steenbergen, "Photoacoustic-guided focused ultrasound (PAFUSion) for identifying reflection artifacts in photoacoustic imaging," Photoacoustics 3(4), 123-131 (2015). 
[18] D. Allman, A. Reiter, and M. A. L. Bell, "Photoacoustic source detection and reflection artifact removal enabled by deep learning," IEEE transactions on medical imaging 37(6), 1464-1477 (2018).

[19] T. Petrosyan, M. Theodorou, J. Bamber, M. Frenz, and M. Jaeger, "Fast scanning widefield clutter elimination in epi-optoacoustic imaging using comb-LOVIT," in Ultrasonics Symposium (IUS), 2017 IEEE International (IEEE, 2017), pp. 1-1.

[20] H.-M. Schwab, and G. Schmitz, "An advanced interpolation approach for photoacoustic clutter reduction based on a linear plane wave scatter model," in Ultrasonics Symposium (IUS), 2016 IEEE International (IEEE, 2016), pp. 1-4.

[21] M. K. A. Singh, M. Jaeger, M. Frenz, and W. Steenbergen, "Photoacoustic reflection artifact reduction using photoacoustic-guided focused ultrasound: comparison between plane-wave and element-by-element synthetic backpropagation approach," Biomed. Opt. Express 8(4), 2245-2260 (2017).

[22] M. K. A. Singh, M. Jaeger, M. Frenz, and W. Steenbergen, "In vivo demonstration of reflection artifact reduction in photoacoustic imaging using synthetic aperture photoacoustic-guided focused ultrasound (PAFUSion)," Biomedical optics express 7(8), 2955-2972 (2016).

[23] V. E. Gusev, and A. A. Karabutov, "Laser optoacoustics," NASA STI/Recon Technical Report A 93, (1991).

[24] A. Oraevsky, and A. Karabutov, "Optoacoustic tomography," Biomedical photonics handbook 34, 1-34 (2003).

[25] A. A. Oraevsky, S. L. Jacques, and F. K. Tittel, "Measurement of tissue optical properties by time-resolved detection of laser-induced transient stress," Applied Optics 36(1), 402-415 (1997).

[26] A. Bashkatov, E. Genina, V. Kochubey, and V. Tuchin, "Optical properties of human skin, subcutaneous and mucous tissues in the wavelength range from 400 to $2000 \mathrm{~nm}$," Journal of Physics D: Applied Physics 38(15), 2543 (2005).

[27] V. V. Tuchin, and V. Tuchin, Tissue optics: light scattering methods and instruments for medical diagnosis (SPIE press Bellingham, 2007). 
[28] M.-L. Li, J.-T. Oh, X. Xie, G. Ku, W. Wang, C. Li, G. Lungu, G. Stoica, and L. V. Wang, "Simultaneous molecular and hypoxia imaging of brain tumors in vivo using spectroscopic photoacoustic tomography," Proceedings of the IEEE 96(3), 481-489 (2008).

[29] I. Sobel, and G. Feldman, "A 3x3 isotropic gradient operator for image processing," a talk at the Stanford Artificial Project in, 271-272 (1968).

[30] J. Benesty, J. Chen, Y. Huang, and I. Cohen, "Pearson correlation coefficient," in Noise reduction in speech processing(Springer, 2009), pp. 1-4.

[31] M. Jaeger, S. Schüpbach, A. Gertsch, M. Kitz, and M. Frenz, "Fourier reconstruction in optoacoustic imaging using truncated regularized inverse k-space interpolation," Inverse Problems 23(6), S51 (2007).

[32] R. Michels, F. Foschum, and A. Kienle, "Optical properties of fat emulsions," Optics Express 16(8), 5907-5925 (2008).

[33] R. Putz, and R. Pabst, Sobotta: Atlas of Human Anatomy (Munich: Elsevier, Urban \& Fischer, 2006).

[34] D. G. Bonett, and T. A. Wright, "Sample size requirements for estimating Pearson, Kendall and Spearman correlations," Psychometrika 65(1), 23-28 (2000). 



\title{
4. Reducing Artifacts in Photoacoustic
} Imaging by Using Multi-Wavelength Excitation and Transducer Displacement $^{3}$

\begin{abstract}
The occurrence of artifacts is a major challenge in photoacoustic imaging. The artifacts negatively affect the quality and reliability of the images. An approach using multiwavelength excitation has previously been reported for in-plane artifact identification. Yet out-of-plane artifacts cannot be tackled with this method. Here we propose a new method using ultrasound transducer array displacement. By displacing the ultrasound transducer array axially, we can de-correlate out-of-plane artifacts with in-plane image features and thus remove them. Combining this new method with the previous one allows us to remove potentially completely both in-plane and out-of-plane artifacts in photoacoustic imaging. We experimentally demonstrate this with experiments in phantoms as well as in vivo.
\end{abstract}

\subsection{Introduction}

Recent research has shown numerous potential clinical applications of photoacoustic imaging (PAI) [1-3]. This imaging technique is based on the photoacoustic (PA) effect. Samples are illuminated using short pulsed laser light. The local absorption of light generates ultrasound (US) waves which are then detected by a US transducer. PA images are reconstructed from the detected signals providing localized information about optical absorption properties of the samples. In clinical applications, the obtained information of

\footnotetext{
3 This chapter has been published as: H. N. Y. Nguyen, and W. Steenbergen, "Reducing artifacts in photoacoustic imaging by using multi-wavelength excitation and transducer displacement," Biomedical Optics Express 10(7), 3124-3138 (2019).
} 
endogenous chromophores such as hemoglobin helps diagnosing early stages of various diseases [2, 4-6].

A typical PAI system basically consists of a light source and US transducer array. The transducer array can be classified as one-dimensional or two-dimensional [7]. While the twodimensional transducer array provides 3D images, it requires significant users' effort and experience for acquiring and interpreting these 3D images [8]. Additionally, twodimensional transducer arrays and the associated scanners are unaffordable for many clinical applications [8]. One-dimensional transducer arrays, in contrast, are widely used for clinical studies $[9,10]$, so from the point of view of clinical translation the incorporation of PAI in a one-dimensional array is preferred.

Several compact and low-cost PAI systems for clinical use have been developed. Integrating a laser source into a handheld US probe stands out among the approaches [9-12]. However, the occurrence of artifacts related to acoustic inhomogeneity of the tissue (clutter) is a major drawback of using a linear US transducer array. The artifacts aimed in this work include in-plane artifacts (IPAs), also called reflection artifacts, and out-of-plane artifacts (OPAs). While IPAs are caused by signals being reflected inside the imaging plane, OPAs are caused by absorbers located outside the imaging plane of the transducer array [13, 14]. These artifacts appear as real image features, such as blood vessels, in the acquired image leading to misinterpretation. Therefore, correcting artifacts in PAI is of importance.

Previously, we proposed a method, photoacoustic-guided focused ultrasound (PAFUSion), to reduce IPAs $[13,15]$. This method has several limitations: a large number of US images are needed; the imaging plane has to be perpendicular to the PA sources; it is limited to reflectors within the angular aperture of the US probe. We then proposed another method using multi-wavelength excitation [14] which can overcome those limitations. In this method, the sample is imaged with laser light at multiple wavelengths to give the PA spectral response of the image features. It is assumed that the spectral response of the IPAs is highly correlated with the true image features of their corresponding real absorbers. Additionally, due to longer propagation of reflected US waves, IPAs appear at larger depths with weaker signals than their corresponding real features. These findings, therefore, can reveal IPAs. However, both this method and PAFUSion do not work for OPAs. 
Several approaches reducing OPAs have been reported such as Deformation Compensated Averaging (DCA) and Localized Vibration Tagging (LOVIT) [16-18]. These approaches employ tissue deformation and motion tracking to de-correlate the OPAs. They can remove almost completely OPAs. However, these methods strongly rely on motion tracking and only work for easily deformable tissue. Additionally, the assumption that OPAs are deformed differently from in-plane features might be incorrect. Furthermore, a large number of US frames is required in these methods.

In this work, we propose a method for suppression of the OPAs by axially displacing the US transducer array. During the displacement, OPAs move up while in-plane features remain at their original position relative to the initial position of the transducer array. Comparing a sequence of images acquired during the displacement can therefore remove OPAs. We then combine this method with our previous work using multi-wavelength excitation to identify and remove both IPAs and OPAs.

We demonstrate the method in phantoms and in vivo. Results show that this is a promising approach for correcting artifacts in PAI.

\subsection{Theory}

Fig. 4.1 illustrates the principle of artifacts in PAI. Fig. 4.1(a) shows a configuration in which several artifacts are present in an acquired image, Fig. 4.1(b). This acquired image represents the imaging plane (orange plane in Fig. 4.1(a)) of the transducer array. There are two absorbers in this configuration: one is in the imaging plane and the other one is outside of the imaging plane. Since the light source excites a large volume, absorber located outside the imaging plane may absorb the light and generate signals. If the sensitivity of the transducer array at that angle is high enough [19], the out-of-plane absorber appears in the acquired image as an OPA, also called direct OPA (downward yellow arrow). In addition, underneath these absorbers are one or more acoustic reflectors. As a consequence, two more artifacts appear in the acquired image: IPA, also called reflection artifact (reflection of the in-plane absorber, upward dashed blue arrow) and indirect OPA (reflection of the out-ofplane absorber, downward dashed yellow arrow). 


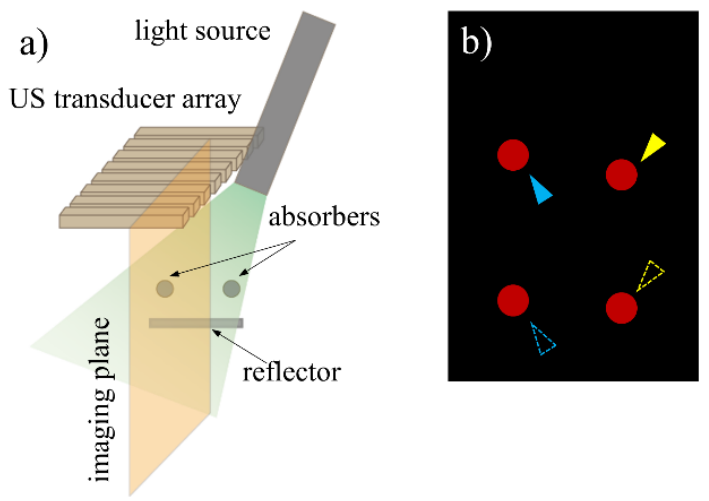

Fig. 4.1. Artifacts in PAI. (a) Configuration resulting in artifacts. (b) Acquired PA image.

Only one feature (upward blue arrow) should be present in the correct PA image of this configuration. However, three more visible features which are artifacts might lead to misinterpretation. In clinical imaging, direct OPAs might come from the skin surface such as human spots and birthmarks. Skin and bone layers which are strong acoustic reflectors can cause indirect OPAs.

\subsection{Method}

\subsubsection{Transducer array displacement}

The principle of this method for identifying OPAs is based on their depth in the acquired image. OPAs appear at depths equal to the distance of their corresponding absorbers to the transducer array. If the transducer array is axially displaced up, in-plane features move down exact the same distance as the displacement, while the OPAs experience in a lesser extent. In the coordinate system of the initial position (before the displacement) of the probe, the inplane features stay at the same position whereas the OPAs move up. By exploiting these different behaviors, OPAs can be differentiated from the in-plane features.

Fig. 4.2 shows a situation of one in-plane and one out-of-plane absorber. $\mathrm{x}, \mathrm{y}$, and $\mathrm{z}$ axes represent elevation, lateral, and axial axes respectively. Fig. 4.2(c) shows a PA image of a configuration depicted in Fig. 4.2(a). The transducer array is then axially shifted up with $\Delta z$ , seen in Fig. 4.2(b). Another PA image is acquired at the new position of the transducer array, Fig. 4.2(d). An OPA is present in these two acquired images. 

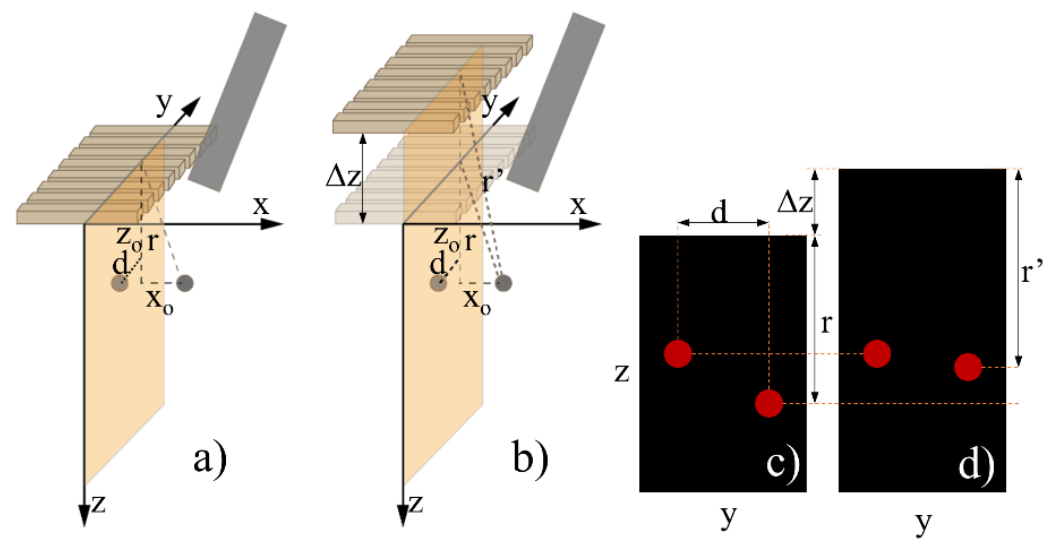

Fig. 4.2. Displacing the transducer array. (a) Initial, and (b) displaced configuration.

(c) Acquired PA image of configuration (a). (d) Acquired PA image of configuration (b).

At the initial position of the transducer array, Fig. 4.2(a), the distance between the outof-plane absorber and the transducer is $\mathrm{r}$. The OPA, therefore, appears at a depth of $\mathrm{r}$ in the acquired image, seen in Fig. 4.2(c). After the transducer array being shifted up, the distance between the out-of-plane absorber and the transducer array is $r^{\prime}$. Since $r^{\prime}<r+\Delta z$, the OPA appears at a smaller depth than in the previous image. However, the in-plane feature stays at the same position. Exploiting these different behaviors, the OPA can therefore be identified and potentially removed.

Denote $s$ as the shift of the OPA. It is determined as $s=r+\Delta z-r^{\prime}$, where $r=\sqrt{x_{o}^{2}+z_{o}^{2}}$ and $r^{\prime}=\sqrt{x_{o}^{2}+\left(z_{o}+\Delta z\right)^{2}} . s$, therefore, is a function of $\Delta z$ and can be rewritten as:

$$
s(\Delta z)=\Delta z+\sqrt{x_{o}^{2}+z_{o}^{2}}-\sqrt{x_{o}^{2}+\left(z_{o}+\Delta z\right)^{2}} .
$$

Images acquired along the displacement are cropped out the extra part from the displacement to have the same size. Assume that they are perfectly segmented to separate features and background. The behavior along transducer array displacement, $\Delta z$, of in-plane and out-of-plane features on the dashed and dotted lines, Fig. 4.3(a), is depicted in Fig. 4.3(b) and (c) respectively. Fig. 4.3(b) and (c) are a sequence of segmented acquired images where blue and yellow represent background and foreground (features). It can be seen that the inplane feature remains at the same position while out-of-plane feature moves up. 
In the segmented images, background and foreground pixels are set as 0 and 1 respectively. Since the OPA moves along $\Delta z$ into the background of the previous images, seen in Fig. 4.3(c), if we multiply segmented images all together the OPA is gradually removed, seen in Fig. 4.3(d). The in-plane feature, in contrast, is not affected. To be able to remove completely the OPA in this way, it is required that the OPA completely moves out of its initial position. At $\Delta z=\Delta z_{T}$ and beyond, the entire OPA is in the background of the initial image, therefore it is completely removed.

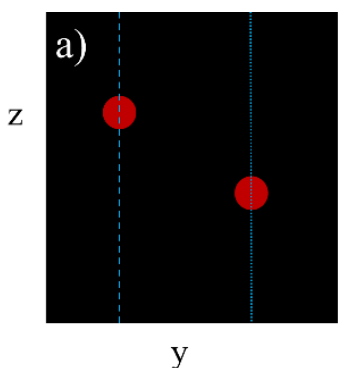

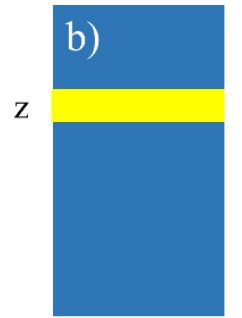

$\Delta \mathrm{z}$

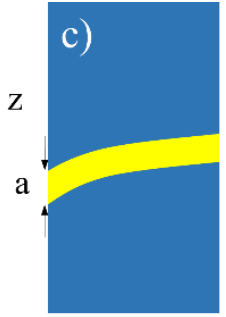

$\Delta \mathrm{z}$

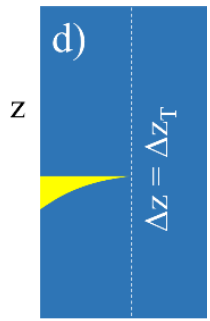

$\Delta \mathrm{z}$

Fig. 4.3. In-plane and out-of-plane features behavior, (b) and (c) on the dotted and dashed lines respectively in (a), as an effect of the transducer array displacement. (d) OPA correcting along the displacement.

To estimate $\Delta z_{T}$, we assume that the axial size of the OPA, $a$ (seen in Fig. 4.3(c)), is a constant during the displacement. The shift of the OPA at $\Delta z=\Delta z_{T}$ must then fulfill $s\left(\Delta z_{T}\right)=a$. Substituting Eq. (4.1) into this gives:

$$
\Delta z_{T}+\sqrt{x_{o}^{2}+z_{o}^{2}}-\sqrt{x_{o}^{2}+\left(z_{o}+\Delta z_{T}\right)^{2}}=a .
$$

Solving this equation, $\Delta z_{T}$ is determined as:

$$
\Delta z_{T}=\frac{2 a \sqrt{x_{o}^{2}+z_{o}^{2}}-a^{2}}{2\left(\sqrt{x_{o}^{2}+z_{o}^{2}}-z_{o}-a\right)} .
$$

Eq. (4.3) holds when the denominator is positive. This equation shows that $\Delta z_{T}$ is dependent on the OPA's axial size, $a$, the distance between the OPA and the imaging plane, $x_{o}$, and the axial distance between the OPA and the transducer array, $z_{o}$. 
Fig. 4.4 illustrates the dependence of $\Delta z_{T}$ on the 3 parameters. It is worth noting that the larger $z_{o}$ is, the larger $\Delta z_{T}$ is; the larger $a$ is, the larger $\Delta z_{T}$ is, seen in Fig. 4.4(a); and the larger $x_{o}$ is, the smaller $\Delta z_{T}$ is, seen in Fig. 4.4(b).
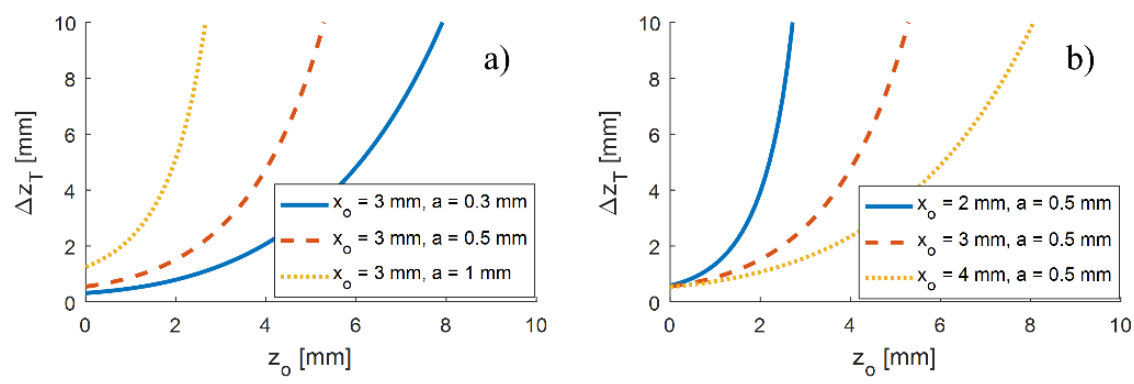

Fig. 4.4. Dependence of $\Delta z_{T}$ on the 3 parameters $z_{o}, a$, and $x_{o}$.

Above is a study for a single OPA. Two images are sufficient for this method (one at the initial position and another one at $\Delta z_{T}$ or beyond) to remove the OPA. In a scenario that there are various OPAs, $\Delta z_{T}$ is then different for different OPAs. In that case, two images might be insufficient. On the other hand, the larger $\Delta z$ is, the more OPAs can be removed. However, at a certain $\Delta z$ when all OPAs are removed, the corrected image does not change despite further displacement since there is no more OPA. At this point, the process can be finished giving the corrected image.

The method is summarized in the following flow chart, Fig. 4.5. The segmentation algorithm, which is based on the Sobel edge detection algorithm [20], and the desegmentation step are the same as presented in [14].

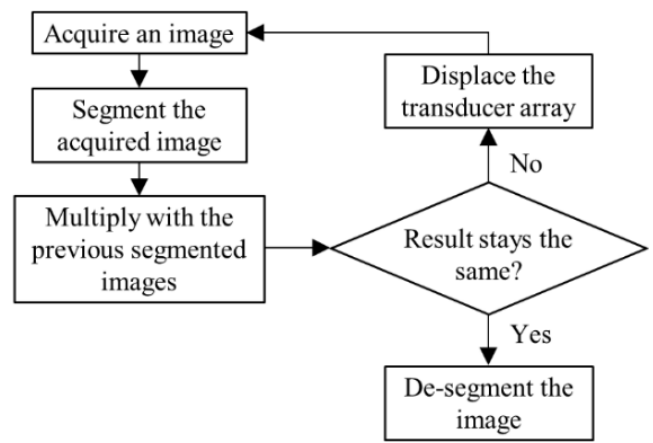

Fig. 4.5. Flow chart of the transducer array displacement method. 
To estimate $\Delta z_{T}$, we have assumed that acquired images are perfectly segmented and the axial size of the OPA does not change during the displacement. However, this is not the case in reality. This will be further discussed in the Discussion section. In addition, the displacement distance, $\Delta z$, might be limited, this will also be addressed in the Discussion section.

\subsubsection{Combining two methods}

We have previously reported a method for identifying IPAs (reflection artifacts) using multi-wavelength excitation [14]. By imaging with multiple wavelengths, spectral responses of features can be obtained. The spectral responses are then correlated to each other using the Pearson correlation coefficient [21]. Based on the correlation coefficients, IPAs can be revealed. However, this method does not work for OPAs. In this work, we combine the previous method with the transducer array displacement method described above as a complete method to remove all artifacts in PAI.

The previous method for IPAs can work with or without segmentation. The approach without segmentation does not rely on the segmentation algorithm, however, it requires significantly more data processing time and expensive calculation. On the other hand, image segmentation is needed in the method for OPAs described above. Therefore, we use segmentation in this work.

It is necessary to remind that in the previous method, of the acquired images with different wavelengths, the image that had the highest signal was selected for segmentation. However, since we combine the two methods, the image acquired at the mutual wavelength is selected for segmentation. The two methods are combined as the following steps:

1. Image with multiple wavelengths $\left(\lambda_{1} \ldots \lambda_{n}\right)$.

2. Image along the transducer array displacement, $\Delta z$, at a fixed wavelength $\lambda_{n}$.

3. Segment the image acquired at $\lambda_{n}, \Delta z=0 \mathrm{~mm}$.

4. Correct IPAs.

5. Correct OPAs giving the final corrected image 


\subsection{Setup}

Fig. 4.6 shows the experimental setup. A handheld US array probe is connected to a commercial US scanner MyLabOne (Esaote Europe BV, The Netherlands) which is presented in [14]. The US transducer array in the handheld probe comprises 128 elements with a pitch of $0.24 \mathrm{~mm}$. It has a center frequency of $7.5 \mathrm{MHz}$ with a bandwidth of $66 \%$. In our study, the central 64 elements were used.

The handheld probe is mounted on a motorized translation stage (MTS50A-Z8, Thorlabs, Germany) which can translate along the axial axis. The light source is an Opolette 532 (Laser2000, The Netherlands) which can emit laser light at a tunable wavelength in two ranges $(680-960 \mathrm{~nm}$ or $1200-2400 \mathrm{~nm})$ at a repetition rate of $20 \mathrm{~Hz}$. Laser light is then coupled into a custom-made multi-mode optical fiber bundle (LightGuideOptics Germany $\mathrm{GmbH}$, Germany) with a core diameter of $6.5 \mathrm{~mm}$. The fiber bundle is fixated. A function generator (AFG 3102, Tektronix, Germany) is used to synchronize the system and to externally trigger the laser. The hardware components are controlled by a LabVIEW program.

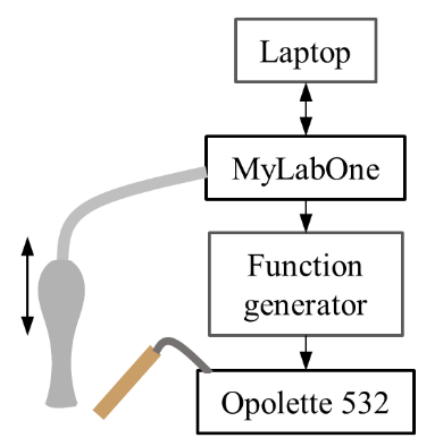

Fig. 4.6. Schematic drawing of the setup with the probe movable in the vertical position and the optical fiber in a fixed position.

In our experiments, laser light at wavelengths in the range of 680-960 nm was used. We used the multi-wavelength method with 8 wavelengths rather than 4 as in [14]. The pulse energy at the output of the fiber was $4.4 \pm 0.4 \mathrm{~mJ}$. 


\subsection{Experimental results}

We performed experiments in both phantoms and in vivo to demonstrate the method. In each experiment, a total number of 13 images were acquired: 1 US image, 8 PA images at 8 different wavelengths $(720: 10: 790 \mathrm{~nm})$, and 5 PA images at a wavelength of $790 \mathrm{~nm}(1$ image at every $1 \mathrm{~mm}$ of total $5 \mathrm{~mm}$ displacement of the transducer array). The US image was reconstructed from 61 angles $\left(-30: 1: 30^{\circ}\right)$ of plane waves. The PA images were averaged over 100 pulses and reconstructed using a Fourier transform based reconstruction algorithm [22].

Since PA images during the transducer array displacement were acquired at a wavelength of $790 \mathrm{~nm}$. The image selected for segmentation in correcting IPAs using multiple wavelengths was at $790 \mathrm{~nm}$ instead of the image giving the highest signal as presented in [14].

Compared to images in our previous study [14], the signal-to-noise ratio is improved due to higher laser pulse energy. In order to present the results, $\mathrm{x}, \mathrm{y}$, and $\mathrm{z}$ axes are used in figures to represent elevation, lateral and axial axes respectively.

\subsubsection{Phantom 1}

A phantom was made of two black absorbers with a thickness of $\sim 0.7 \mathrm{~mm}$ cut out from a black thread embedded in agarose (1.5\%) in a petri dish lid (Greiner Bio-One GmbH, Germany), as shown in Fig. 4.7(a). The petri dish lid of $750 \mu m$ thickness was used as an acoustic reflector. Fig. 4.7(b) is a schematic elevation view of the experiment configuration. One absorber was placed underneath the probe and the other one was positioned $\sim 3-4 \mathrm{~mm}$ off the probe's edge to make sure it was outside of the imaging plane. The coupling medium was a suspension of $2 \%$ Intralipid $20 \%$ in demi-water with estimated $\mu_{s}^{\prime}=4.2 \mathrm{~cm}^{-1}$ at a wavelength of $750 \mathrm{~nm}$ [23] mimicking scattering in human soft tissue [24]. 

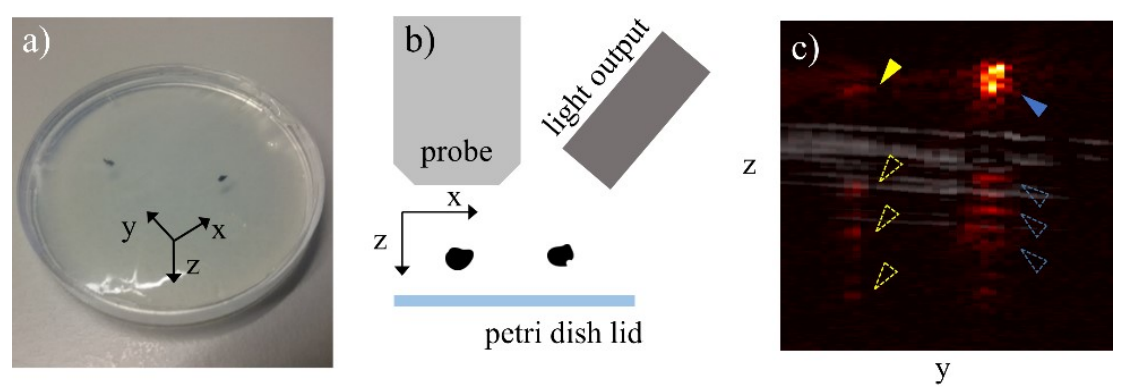

Fig. 4.7. phantom 1. (a) Two absorbers embedded in agarose in a petri dish lid. (b) Schematic elevation view of the experiment configuration. (c) Combined PA and US image.

A combined PA and US image is shown in Fig. 4.7(c). The gray color part represents the US image showing surfaces and reverberations of the petri dish lid. The hot color part is the PA image where the in-plane absorber (upward blue arrow) is visualized at an expected position relative to the lid. Underneath the lid are some more image features (upward dashed blue arrow), which are IPAs of the in-plane absorber. A direct OPA (downward yellow arrow) is also visible at an expected position relative to the lid and the in-plane absorber. At the bottom left corner of the image are some features (downward dashed yellow arrow) which are probably indirect OPAs of the out-of-plane absorber.

Fig. 4.8 shows the result of correcting IPAs using 8 wavelengths. Most of the IPAs (reflections of the in-plane absorber) are removed. However, some parts of the reflections are retained (dashed blue arrows, seen in Fig. 4.8(b)). The reason would be that the spectral response of these features is strongly affected by noise which was also pointed out in [14].
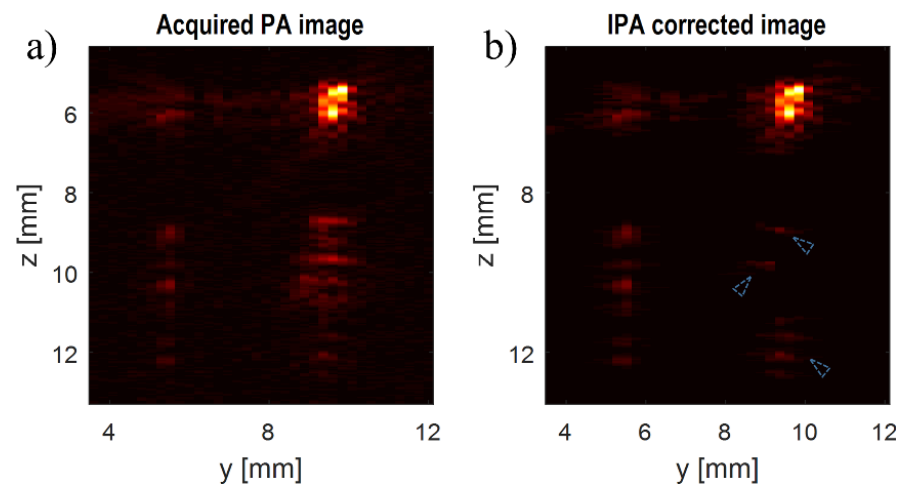

Fig. 4.8. Correcting IPAs. (a) Acquired PA image. (b) IPA corrected image. 
The acquired image was then OPA corrected with displacing the transducer array. Fig. 4.9(b) and (c) respectively show the behavior of the out-of-plane and in-plane features marked with a dotted line and a dashed line in Fig. 4.9(a) during the displacement, seen in Appendix 2.3.
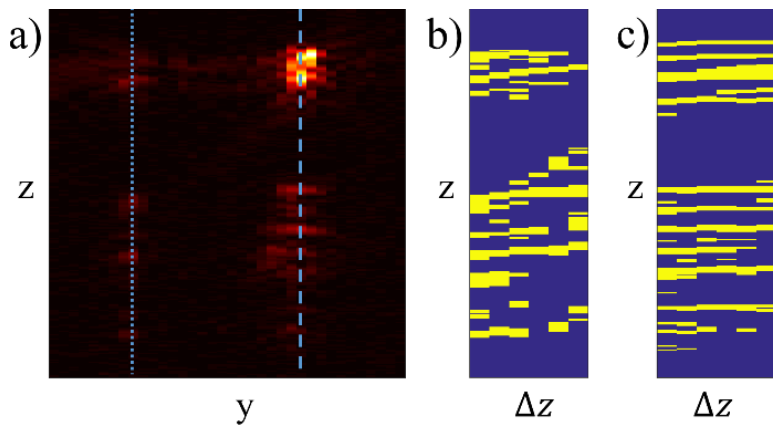

Fig. 4.9. In-plane and out-of-plane features behavior, (b) and (c) on the dotted and dashed lines respectively in (a), as an effect of the transducer array displacement.

The out-of-plane features clearly shift up, especially the direct OPA. The shift of indirect OPAs is less pronounced since they have a larger axial distance to the transducer array. The size of in-plane features can be seen changing due to the axial resolution of the transducer array along depth. In addition, the position of in-plane features also slightly changes. The reason might be that the in-plane absorber was slightly off the imaging plane or the displacement was not perfectly along the axial axis or displacing the transducer array produced some positioning error.

Fig. 4.10 presents the result of correcting OPAs with $5 \mathrm{~mm}$ transducer array displacement, see also in Appendix 2.4. An OPA (dashed yellow arrow, seen in Fig. 4.10(b)) is not completely removed showing that $\Delta z_{T}$ should be larger than $5 \mathrm{~mm}$ for this OPA to be completely removed. Additionally, as there is some slight movement of in-plane features, part of them is consequently overcorrected. 

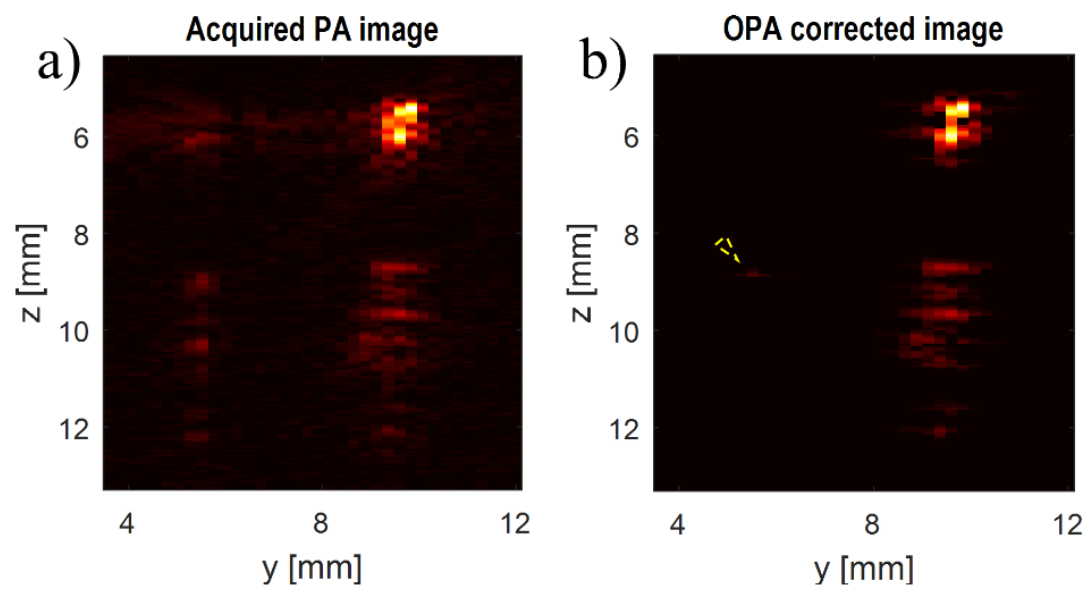

Fig. 4.10. Correcting OPAs. (a) Acquired PA image. (b) OPA corrected image.

Fig. 4.11 shows the result of combining the two methods for IPA and OPA. Compared to the acquired PA image, Fig. 4.11(a), most of all artifacts are identified and removed giving the final corrected image with only one true in-plane absorber, seen in Fig. 4.11(b).
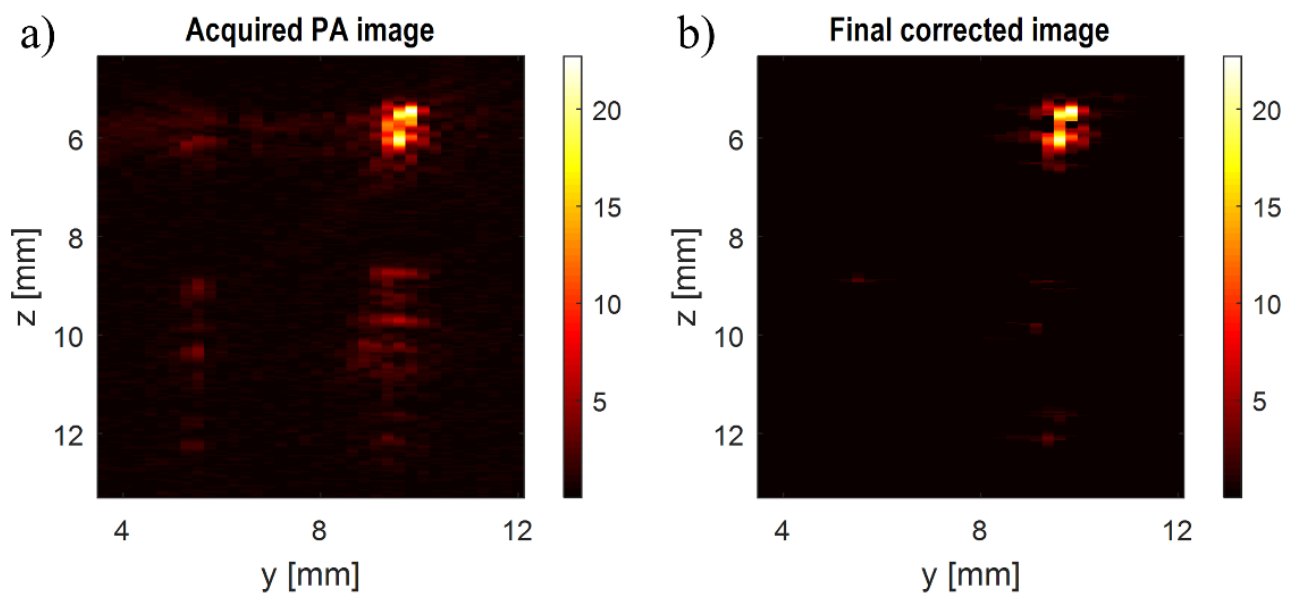

Fig. 4.11. Final corrected image.

The displacing transducer array method does not work for IPAs. In this experiment, a few more IPAs are removed using this method due to their slight movement during the displacement. 


\subsubsection{Phantom 2}

Another phantom was made to mimic a situation that an OPA appears at the same position with an in-plane feature. Two black absorbers (the same as in phantom 1) were embedded in agarose (1.5\%) in a petri dish, Fig. 4.12(a). A solution of $2 \%$ Intralipid $20 \%$ in demi-water was used as the coupling medium. The two absorbers were placed under the probe as in Fig. 4.12(b) where one absorber was in the imaging plane and the other one was outside of the imaging plane and the distance of these absorbers to the probe was the same. As a consequence, in the acquired PA image, the two absorbers appeared at the same position.

Fig. 4.12(c) shows the PA images during the transducer array displacement. At the initial position, $\Delta z=0 \mathrm{~mm}$, only one feature is visible. The curve at the same position of this feature is a reconstruction artifact. When the probe is lifted up, $\Delta z>0 \mathrm{~mm}$, another feature starts to appear and move up along with the displacement while the in-plane feature remains at the same position, seen in Appendix 2.5.

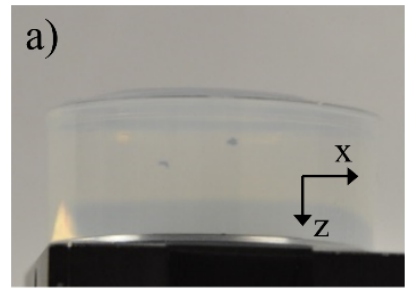

b)

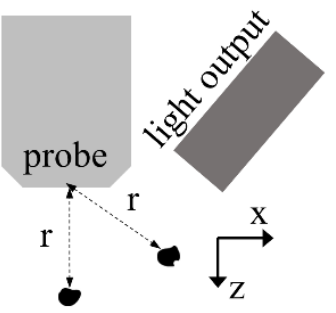

c)
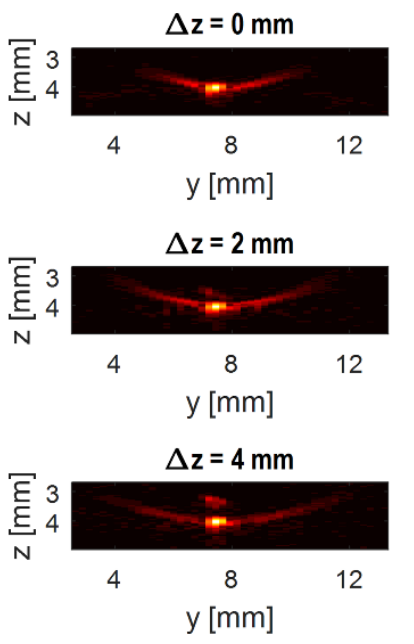
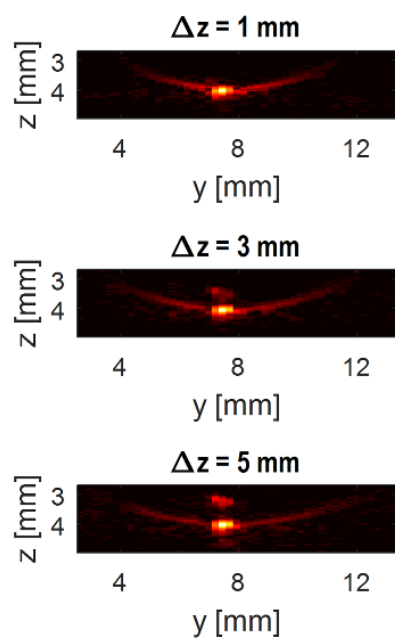

Fig. 4.12. Phantom 2. (a) Two black absorbers embedded in agarose in a petri dish. (b) Schematic elevation view of the experiment configuration. (c) Acquired images along the displacement.

Fig. 4.13(a) presents the result of this experiment where the OPA is completely removed. The OPA, as expected, moves up away from the real in-plane feature which stays 
at the same position. These behaviors can be seen clearly in Fig. 4.13(b) which shows the dashed blue line in the acquired image. Similarly, Fig. 4.13(c) illustrates the behavior of the reconstruction artifact marked in the dotted blue line in the acquired image. It is worth noting that this type of reconstruction artifact is dependent on the distance to the probe. Since it becomes less curved, its part in the dotted line moves down. As a result, this reconstruction artifact is partly removed, seen in Fig. 4.13(a).
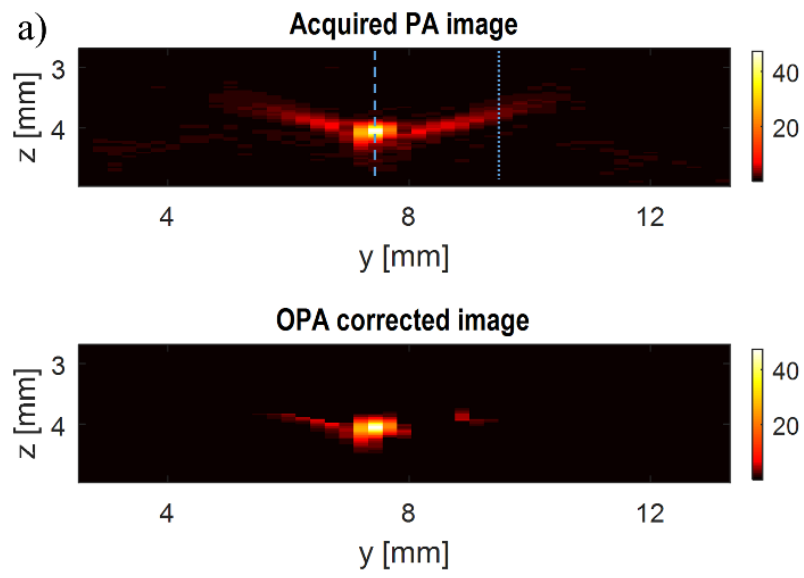

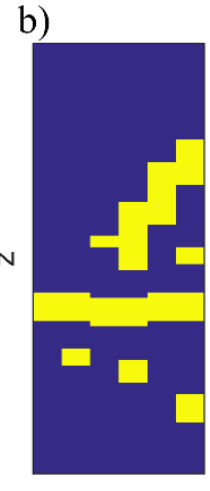

$\Delta z$

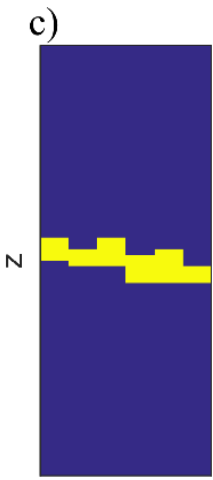

$\Delta z$

Fig. 4.13. OPA corrected image. (a) Acquired and OPA corrected images. (b) and (c) Behavior of features in dashed and dotted lines in (a) respectively.

It is notable that when an OPA appears at the same position with a real in-plane feature as a single feature, the recorded amplitude of that overlapped feature is a sum of the amplitudes of the two features. Though the OPA can be removed, the true amplitude of the real in-plane feature cannot be recovered. This will be discussed further in the Discussion section.

\subsubsection{In vivo}

We also assess our method with in vivo experiments. In these in vivo experiments, we aimed at fingers which would give clear IPAs. In addition, we put a black ink mark on the skin of the imaged finger to mimic a human spot which would give strong OPAs [25].

Fig. 4.14 shows the configuration of the in vivo experiments. A volunteer's finger with an ink mark was placed in a water tank filled with demi-water as a coupling medium, Fig. 
4.14(a). The white line in Fig. 4.14(b) depicts approximately the imaging plane in the experiments. The ink mark was a few millimeters outside of the imaging plane.

Acquired PA and US images are presented in Fig. 4.14(c) and Fig. 4.14(d) respectively. In principle, they show similar structures as observed in [14] where the US image shows the skin and bone layers while the PA image shows the skin, superficial blood vessels and IPAs. However, in this PA image, there are a few more features (dashed blue circle) appearing at the position in agreement with the ink mark's position on the skin surface. These are probably OPAs.
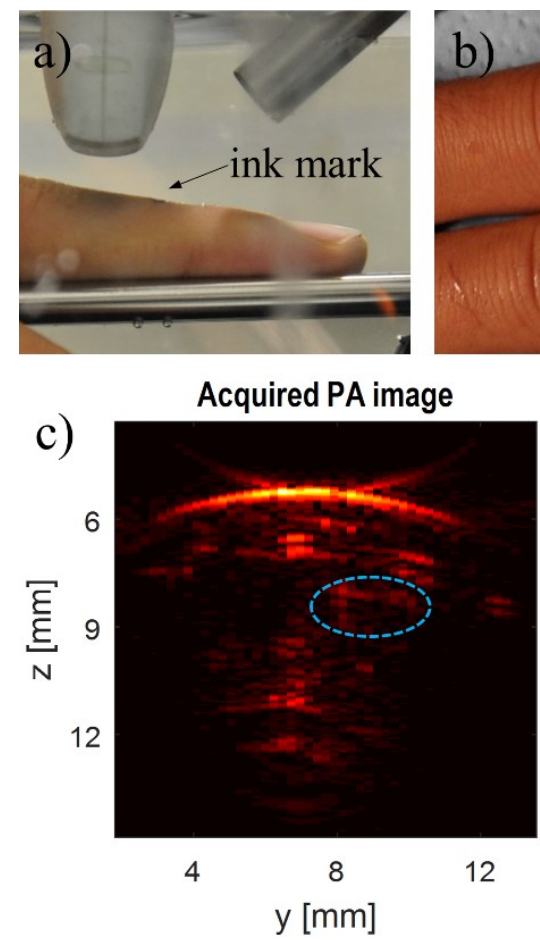
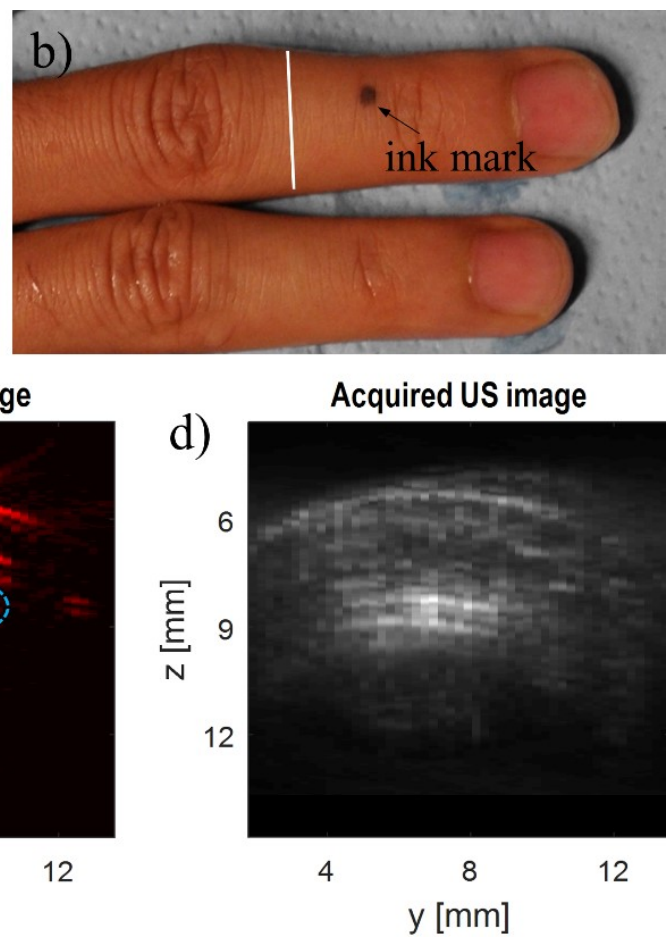

Fig. 4.14. In vivo experiment. (a) Experiment configuration. (b) Ink mark mimicking a human spot. (c) Acquired PA image. (d) Acquired US image.

Fig. 4.15 shows IPA and OPA corrected images. In the IPA corrected image, Fig. 4.15(b), most of the IPAs caused by the bone layers are removed. This matches with the results reported in [14]. Two features, $\mathrm{fl}$ and $\mathrm{f} 2$ in Fig. 4.15(a), are of interest. Observation from correcting OPA shows that $\mathrm{f1}$ is an OPA and $\mathrm{f} 2$ is an IPA (seen in Appendix 2.6, $\mathrm{f1}$ moves up and $\mathrm{f} 2$ remains at the same position). $\mathrm{fl}$ could be an indirect OPA of the ink mark 68 
reflected by the skin, while 2 could be a reflection of the skin signal. However, $\mathrm{fl}$ is removed while $\mathrm{f} 2$ is not, in the IPA corrected image. The reason can be that the intensity of these two features is close to the noise. On the other hand, there are some OPAs at the same position with $\mathrm{f} 2$. This can be seen in Appendix 2.6 in which some features move up away from $\mathrm{f} 2$ during the transducer array displacement. Therefore, the true intensity of $f 2$ is affected by these features, as mentioned in the phantom 2 experiment, and thus it is not identified as an IPA. The reconstruction artifact curve of the skin is also removed with the OPA correcting method. In addition, some real in-plane features are party removed in the OPA corrected image. The reason is that there was some slight movement of the finger during the transducer array displacement.
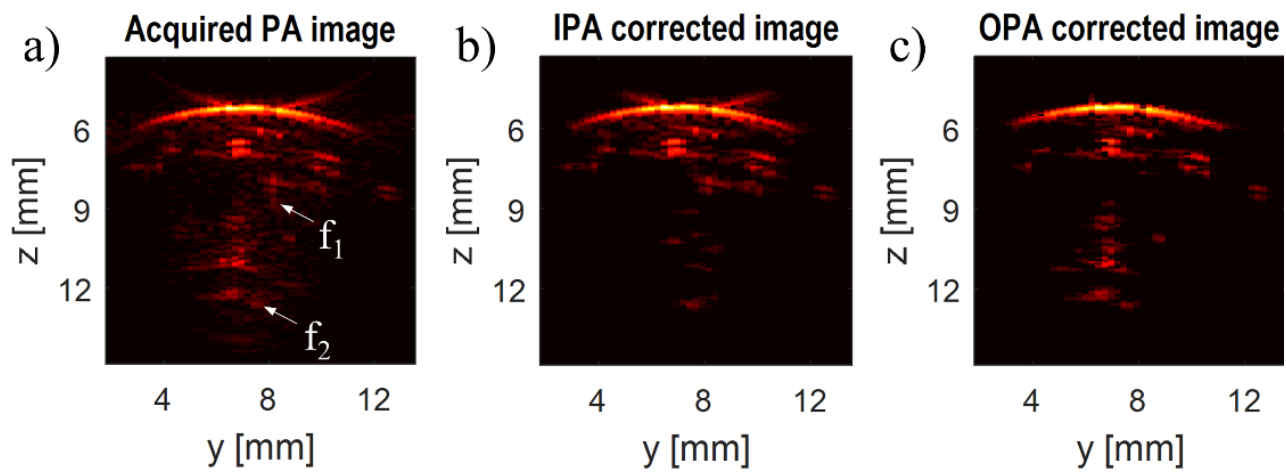

Fig. 4.15. IPA and OPA corrected images.

Combing correcting IPA and OPA gives the final corrected image, Fig. 4.16.
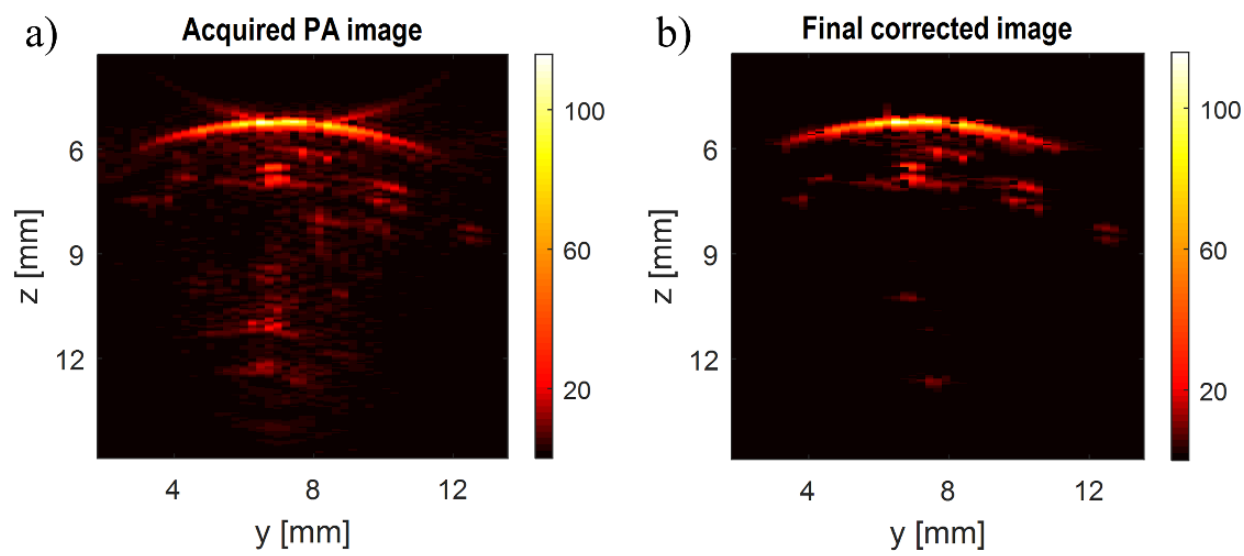

Fig. 4.16. Final corrected image. 


\subsection{Discussion}

Correcting artifacts is of importance for reliable imaging. Our new method for OPAs offers various advantages over previously reported methods. First of all, no motion tracking is needed as in the case of LOVIT and DCA [16-18]. Second of all, these existing methods require a large number of US images while no US image is needed in the proposed method. Third of all, deforming tissue by applying force might affect the optical properties of the tissue as in DCA. As a consequence, the detected signals might not represent truly the source. In the proposed method, correcting OPAs can be done without tissue deformation. Lastly, deforming tissue by focusing strong pressure, as is done in LOVIT, might violate US safety.

The proposed method for correcting OPA relies on segmentation. In this work, a simple segmentation approach was used for a low calculation cost and time consumption. However, it might not segment images properly giving inaccurate axial dimension of OPAs. As a result, OPAs might not be correctly removed. Over-segmentation also might happen as pointed out in [14]. A more effective segmentation algorithm should be considered for a better performance.

In our experiments, while the probe was displaced, the fiber bundle remained fixated. The purpose of this was to maintain the signal strength of image features. However, if the light source is displaced with the probe, the laser beam is also repositioned and thus excites different tissue volumes. Acquired images along the displacement might show different structures resulting in miscorrecting.

In clinical applications, the displacement distance, $\Delta z$, might be limited. Depending on the location of out-of-plane absorbers, $\Delta z_{T}$ might not be achieved, as discussed in section 4.3.1. As a result, OPAs are not completely removed, in which case another approach is needed. However, our results show that within $5 \mathrm{~mm}$ displacement, OPAs can be completely removed for a large range of locations and axial dimensions.

In this work, out-of-plane absorbers were positioned outside of the imaging plane elevationally. Lateral out-of-plane absorbers can also cause OPAs. The principle of the proposed method still holds for these OPAs. The quantity $x_{o}$, used to describe out-of-plane absorbers in section 4.3.1, in this situation will be the lateral distance between the out-of- 
plane absorber and the imaging plane. Therefore, lateral OPAs can be identified and removed.

Axially displacing the probe in essence is to adjust the distance between the probe and in-plane and out-of-plane absorbers. Displacing the probe in other directions might also be able to de-correlate in-plane and out-of-plane image features. In a configuration as shown in phantom 1, if the probe is elevationally displaced in the direction to the out-of-plane absorber, real in-plane features will move down and OPAs will move up. However, in a scenario that there is another out-of-plane absorber in the other side of the imaging plane. OPAs of this out-of-plane absorber will also move down. Therefore, elevationally displacing the probe in both directions is required to identify all OPAs. The amount of work is double compared to using axial displacement. Nevertheless, displacing the probe in other manners and comparing with the proposed method will be investigated in our future work.

In a situation that an OPA appears at the same position with a real in-plane feature as a single feature, the image value is a sum of the OPA and the in-plane features. Displacing the transducer array can separate these two and remove the OPA. However, true image value of the real in-plane feature cannot be recovered. Interpolating or extrapolating image values of the OPA along the displacement might be able to estimate its value at the superposition. True image value of the real in-plane feature can, therefore, be recovered. Additionally, in the proposed method, OPAs are removed by setting their pixel values to 0 . This might also remove the background information behind the OPAs. If the image value of the OPAs can be estimated, the background information can be retained while removing OPAs by subtracting the recorded value by the estimated one. This will be investigated in our future work.

In this work, the volunteer had to keep the finger still for $\sim 5$ minutes. Slight movements were inevitable resulting in some miscorrection. This is not ideal for clinical applications. However, the long experiment time was due to technical limitations. In particular, the translating stage was slow. It took $\sim 2$ minutes to acquire in total 5 PA images along $5 \mathrm{~mm}$ of the probe displacement. Using a higher speed translating stage will significantly reduce the experiment time. The acquiring data process with 8 wavelengths took $\sim 2$ minutes. This was due to the laser pulse repetition rate of $20 \mathrm{~Hz}$. Using a high repetition rate laser would potentially achieve real-time artifact correction as shown in [14]. 


\subsection{Conclusion}

We have proposed a new method to remove out-of-plane artifacts exploiting different behaviors of out-of-plane artifacts and in-plane image features by axially displacing the transducer array. Combining this new method with our previous method for in-plane artifacts using multiple wavelengths [14], in-plane and out-of-plane artifacts in photoacoustic imaging can be identified and thus removed. Experiments in phantoms and in vivo were carried out to evaluate the combination of the two methods as a proof of concept. Results show the potential of this combined method for providing true photoacoustic images with no ultrasound images needed. In addition, a handheld probe suitable for clinical applications was used in the experiments bringing this method a step forwards to clinical translation. 


\subsection{References}

[1] K. S. Valluru, K. E. Wilson, and J. K. Willmann, "Photoacoustic Imaging in oncology: translational preclinical and early clinical experience," Radiology 280(2), 332-349 (2016).

[2] M. Heijblom, D. Piras, F. M. van den Engh, M. van der Schaaf, J. M. Klaase, W. Steenbergen, and S. Manohar, "The state of the art in breast imaging using the Twente Photoacoustic Mammoscope: results from 31 measurements on malignancies," Eur. Radiol. 26(11), 3874-3887 (2016).

[3] M. Heijblom, W. Steenbergen, and S. Manohar, "Clinical photoacoustic breast imaging: the twente experience," IEEE Pulse 6(3), 42-46 (2015).

[4] P. J. van den Berg, K. Daoudi, H. J. B. Moens, and W. Steenbergen, "Feasibility of photoacoustic/ultrasound imaging of synovitis in finger joints using a point-of-care system," Photoacoustics 8, 8-14 (2017).

[5] M. Toi, Y. Asao, Y. Matsumoto, H. Sekiguchi, A. Yoshikawa, M. Takada, M. Kataoka, T. Endo, N. Kawaguchi-Sakita, and M. Kawashima, "Visualization of tumor-related blood vessels in human breast by photoacoustic imaging system with a hemispherical detector array," Sci. Rep. 7, (2017).

[6] J. Jo, G. Xu, M. Cao, A. Marquardt, S. Francis, G. Gandikota, and X. Wang, "A Functional Study of Human Inflammatory Arthritis Using Photoacoustic Imaging," Sci. Rep. 7(1), 15026 (2017).

[7] B. W. Drinkwater, and P. D. Wilcox, "Ultrasonic arrays for non-destructive evaluation: A review," NDT\&E. Int 39(7), 525-541 (2006).

[8] C. D. Herickhoff, M. R. Morgan, J. S. Broder, and J. J. Dahl, "Low-cost volumetric ultrasound by augmentation of 2D systems: Design and prototype," Ultrasonic imaging 40(1), 35-48 (2018).

[9] M. K. A. Singh, W. Steenbergen, and S. Manohar, "Handheld probe-based dual mode ultrasound/photoacoustics for biomedical imaging," in Frontiers in Biophotonics for Translational Medicine(Springer, 2016), pp. 209-247. 
[10] K. Daoudi, P. Van Den Berg, O. Rabot, A. Kohl, S. Tisserand, P. Brands, and W. Steenbergen, "Handheld probe integrating laser diode and ultrasound transducer array for ultrasound/photoacoustic dual modality imaging," Opt. Express 22(21), 2636526374 (2014).

[11] C. Kim, T. N. Erpelding, L. Jankovic, M. D. Pashley, and L. V. Wang, "Deeply penetrating in vivo photoacoustic imaging using a clinical ultrasound array system," Biomed. Opt. Express 1(1), 278-284 (2010).

[12] C. Haisch, K. Eilert-Zell, M. M. Vogel, P. Menzenbach, and R. Niessner, "Combined optoacoustic/ultrasound system for tomographic absorption measurements: possibilities and limitations," Anal. Bioanal. Chem. 397(4), 1503-1510 (2010).

[13] M. K. A. Singh, and W. Steenbergen, "Photoacoustic-guided focused ultrasound (PAFUSion) for identifying reflection artifacts in photoacoustic imaging," Photoacoustics 3(4), 123-131 (2015).

[14] H. N. Y. Nguyen, A. Hussain, and W. Steenbergen, "Reflection artifact identification in photoacoustic imaging using multi-wavelength excitation," Biomed. Opt. Express 9(10), 4613-4630 (2018).

[15] M. K. A. Singh, M. Jaeger, M. Frenz, and W. Steenbergen, "In vivo demonstration of reflection artifact reduction in photoacoustic imaging using synthetic aperture photoacoustic-guided focused ultrasound (PAFUSion)," Biomed. Opt. Express 7(8), 2955-2972 (2016).

[16] M. Jaeger, L. Siegenthaler, M. Kitz, and M. Frenz, "Reduction of background in optoacoustic image sequences obtained under tissue deformation," J. Biomed. Opt. 14(5), 054011 (2009).

[17] T. Petrosyan, M. Theodorou, J. Bamber, M. Frenz, and M. Jaeger, "Fast scanning widefield clutter elimination in epi-optoacoustic imaging using comb-LOVIT," in Ultrasonics Symposium (IUS), 2017 IEEE International (IEEE, 2017), pp. 1-1.

[18] M. Jaeger, J. C. Bamber, and M. Frenz, "Clutter elimination for deep clinical optoacoustic imaging using localised vibration tagging (LOVIT)," Photoacoustics 1(2), 19-29 (2013). 
[19] M. F. Beckmann, H.-M. Schwab, and G. Schmitz, "Optimizing a single-sided reflection mode photoacoustic setup for clinical imaging," in Ultrasonics Symposium (IUS), 2015 IEEE International (IEEE, 2015), pp. 1-4.

[20] I. Sobel, and G. Feldman, "A 3x3 isotropic gradient operator for image processing," a talk at the Stanford Artificial Project in, 271-272 (1968).

[21] J. Benesty, J. Chen, Y. Huang, and I. Cohen, "Pearson correlation coefficient," in Noise Reduction in Speech Processing(Springer, 2009), pp. 1-4.

[22] M. Jaeger, S. Schüpbach, A. Gertsch, M. Kitz, and M. Frenz, "Fourier reconstruction in optoacoustic imaging using truncated regularized inverse k-space interpolation," Inverse Probl. 23(6), S51 (2007).

[23] R. Michels, F. Foschum, and A. Kienle, "Optical properties of fat emulsions," Opt. Express 16(8), 5907-5925 (2008).

[24] S. L. Jacques, "Optical properties of biological tissues: a review," Phys. Med. Biol. 58(11), R37 (2013).

[25] S. Preisser, G. Held, H. G. Akarçay, M. Jaeger, and M. Frenz, "Study of clutter origin in in-vivo epi-optoacoustic imaging of human forearms," J. Opt. 18(9), 094003 (2016). 



\title{
5. Three-dimensional View of Out-of-
}

\author{
plane Artifacts in Photoacoustic \\ Imaging Using a Laser-integrated
}

Linear-transducer-array Probe ${ }^{4}$

\begin{abstract}
Research on photoacoustic imaging (PAI) using a handheld integrated photoacoustic probe has been a recent focus of clinical translation of this imaging technique. One of the remaining challenges is the occurrence of out-of-plane artifacts (OPAs) in such a probe. Previously, we proposed a method to identify and remove OPAs by axially displacing the transducer array. Here we show that besides the benefit of removing OPAs from the imaging plane, the proposed method can provide a three-dimensional (3D) view of the OPAs. In this work, we present a 3D reconstruction method using axial transducer array displacement. By axially displacing the transducer array, out-of-plane absorbers can be three-dimensionally visualized at an elevation distance of up to the acquired imaging depth. Additionally, OPAs in the in-plane image are significantly reduced. We experimentally demonstrate the method with phantom and in vivo experiments using an integrated PAI probe. We also compare the method with elevational transducer array displacement and take into account the sensitivity of the transducer array in the $3 \mathrm{D}$ reconstruction.
\end{abstract}

\subsection{Introduction}

Photoacoustic imaging (PAI) is a promising medical imaging technique [1-5] thanks to its advantages over conventional techniques. PAI provides images with clinically sufficient imaging depth, high resolution, and optical contrast. It uses short pulsed laser light to

\footnotetext{
${ }^{4}$ This chapter has been published as: H. N. Y. Nguyen, and W. Steenbergen, "Three-dimensional view of outof-plane artifacts in photoacoustic imaging using a laser-integrated linear-transducer-array probe," Photoacoustics, 100176 (2019).
} 
illuminate the sample and detects the ultrasound (US) waves generated by optically absorbing substances. Photoacoustic (PA) images are then formed from the detected signals. The reconstructed images provide localized information of the presence of substances like oxy- and deoxyhemoglobin, melanin and lipids, also depending on the optical wavelength used. In clinical applications, such information may help to diagnose various diseases in early stages $[4,6-8]$.

A conventional PAI system is mechanically bulky and relatively expensive. Reducing the size and cost is necessary for this technique to find a place in clinics. Integrating a low cost laser source, such as laser diodes, into a handheld US probe is an outstanding alternative [9-14]. However, one of the main drawbacks of such a system is the occurrence of out-ofplane artifacts (OPAs), also called clutter [15-18]. In in vivo imaging, OPAs appear as spurious objects in the imaging plane [18] which might lead to wrong conclusions. Correcting OPAs is therefore of importance.

Recently, we proposed a method of using transducer array displacement to identify and remove OPAs $[18,19]$. In this method, the transducer array is displaced axially while the sample and light source are fixated. Consequently, OPAs move along the displacement while in-plane image features remain at the same position. OPAs therefore can be identified and removed.

Here we demonstrate that though the imaging plane remains the same during the axial displacement, this method still can form a volumetric image providing a three-dimensional (3D) view of the OPAs, even with an axial displacement of a few millimeters only. This is a new advantage of our proposed method [18]. In fact, 3D imaging has been a focus in PAI research by using a $2 \mathrm{D}$ transducer array [20,21] or scanning a $1 \mathrm{D}$ transducer array [22, 23]. Using a 2D transducer array requires considerable effort and experience of the user for image acquisition and interpretation. [24]. In addition, a system with a 2D transducer array is complex making it clinically unaffordable [24]. Using a 1D transducer array might overcome those limitations, however, scanning the array in the elevation direction is needed to form a $3 \mathrm{D}$ image. As a consequence, the field of view is then limited within the scanning distance.

In the previous work [18], image segmentation is used in order to identify moving features along the displacement as OPAs. The OPAs are then removed by setting their 
corresponding image pixels to 0 . In this way, detected OPAs are completely removed out of the image however, background information behind the OPAs is also lost [18]. In our 3D reconstruction method presented here, OPAs are reduced in the in-plane image while the background information still remains.

To demonstrate the method, we used a handheld PAI probe showing potential benefit of this method to clinical use. We then compared this method, in terms of resolutions of inplane and out-of-plane absorbers and signal-to-noise (SNR) ratio, with 3D imaging with elevational displacement of the transducer array. We also demonstrated it with in vivo experiments. Additionally, we improved the reconstruction with sensitivity compensation of the transducer array.

\subsection{Method}

We use $\mathrm{x}, \mathrm{y}$, and $\mathrm{z}$ to represent the elevation, lateral, and axial direction, respectively.

\subsubsection{D reconstruction based on direct back-projection}

Normally, data acquired by a $1 \mathrm{D}$ transducer array provides a 2D image representing the imaging plane of the transducer array. However, out-of-plane absorbers might appear in that reconstructed image [17-19]. A 1D transducer array, therefore, actually provides additional information outside of the imaging plane.

Fig. 5.1 presents a configuration of imaging an out-of-plane absorber and steps of forming a 3D projection. Fig. 5.1(a) shows a schematic drawing of a configuration in which a black nylon absorber is placed elevationally outside the imaging plane but still inside the light beam. Particularly, it is positioned $\sim 3.5 \mathrm{~mm}$ axially and $\sim 6.5 \mathrm{~mm}$ elevationally away from the transducer array. Demi-water is used as a coupling medium. The transducer array is in the direction perpendicular to the drawing plane. The photoacoustic imaging system is described in [18]. Fig. 5.1(b) is a reconstructed pressure distribution image (before the envelope detection) in the yz plane, using a Fourier transform based reconstruction algorithm [25]. An OPA is clearly visible in this image. Fig. 5.1(c) depicts the axial profile of the dashed red line in Fig. 5.1(b). This axial line is then back-projected in its own xz plane, seen in Fig. 5.1(d). A 3D projection is formed by back-projecting in the xz plane for all lines in the reconstructed pressure distribution image. 

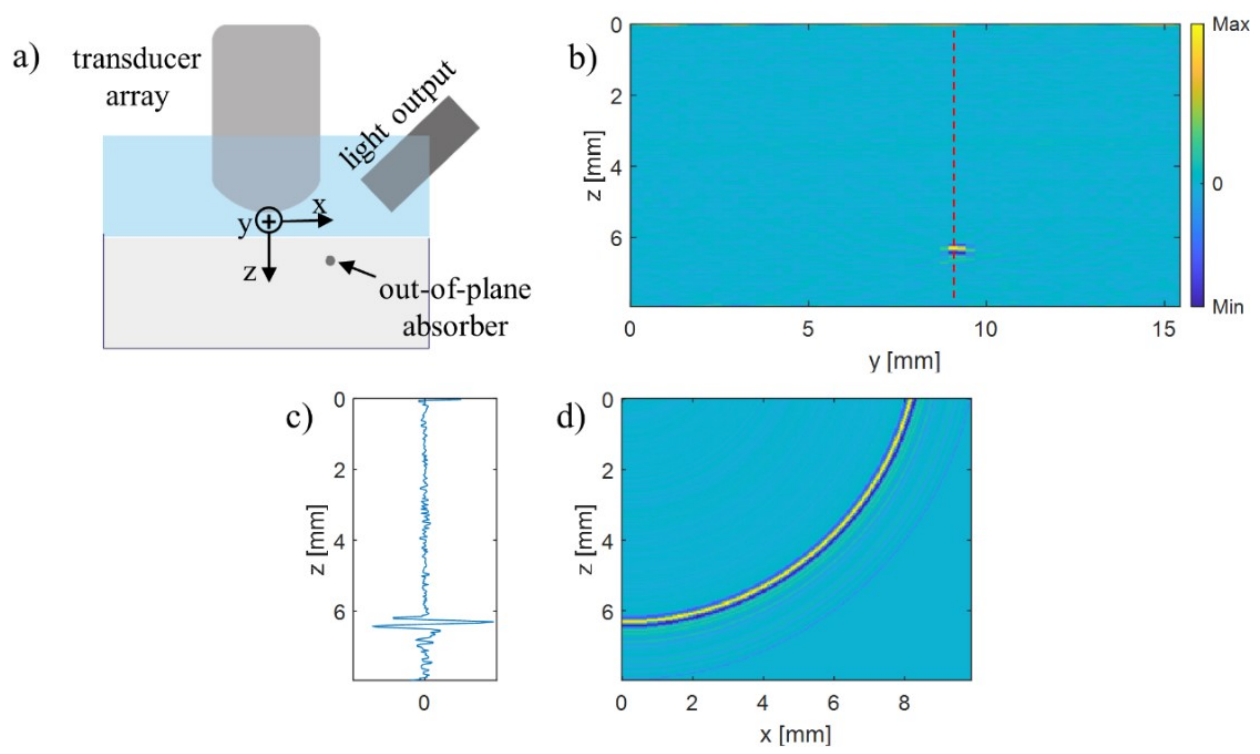

Fig. 5.1. (a) Configuration for imaging an out-of-plane absorber. (b) Pressure distribution image. (c) Axial profile of the dashed red line in (b). (d) xz projection.

The probe is then axially displaced to a new position. A new 3D projection in the same ROI as the original one is formed at the new position, in the same way as described above. Fig. 5.2(a)-(b) present 2 axial lines of the original and new projection respectively. The signal from the out-of-plane absorber clearly moves up. These $23 \mathrm{D}$ projections are summed up together. Fig. 5.2(c) shows an xz image of the sum in which back-projected signals intersect at the position in agreement with the location of the out-of-plane absorber. Fig. 5.2(d) shows an image, in the same xz plane, of the sum of $63 \mathrm{D}$ projections. In the end, envelope detection is performed using the Hilbert transform providing a reconstructed 3D image. Fig. 5.2(e) shows a Hilbert transformed xz image of the reconstructed 3D image. 


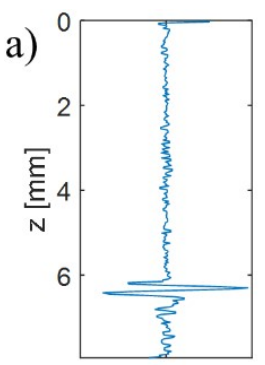

0 b)

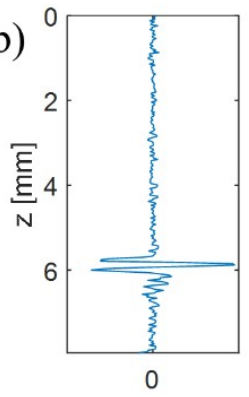

d)

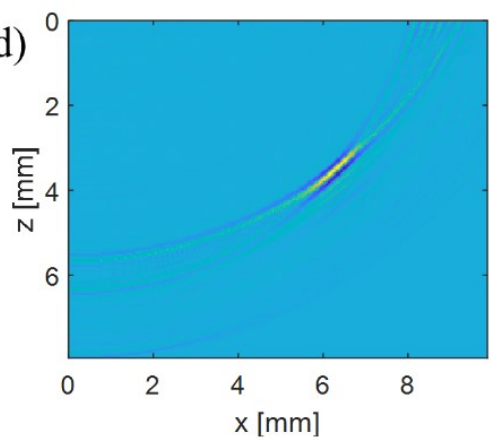

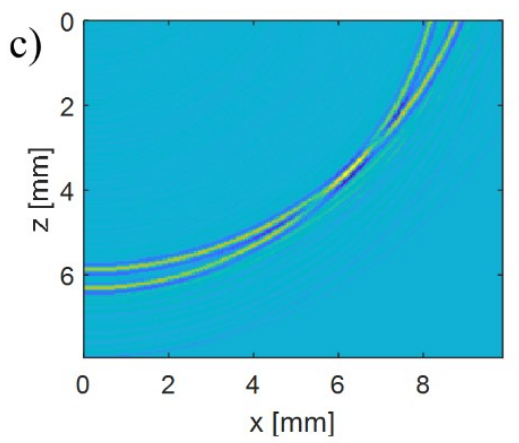

e)

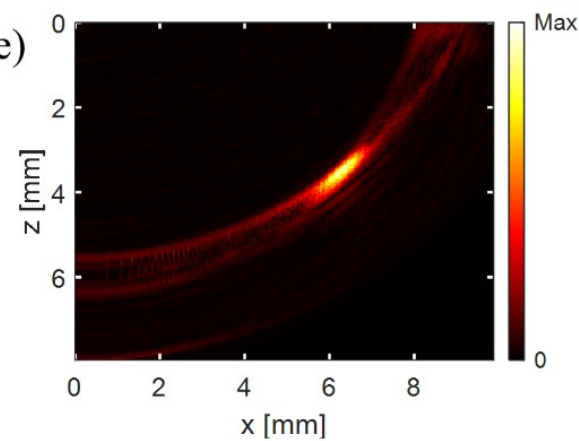

Fig. 5.2.3D reconstruction based on direct back-projection. Axial profile at the original position (a) and a new position (b) of the probe. xz image of the sum of 2 (c) and 6 (d) 3D projections. (e) Hilbert transformed image.

A 3D projection can also be formed by back-projecting the RF signal of all transducer elements volumetrically. This approach gives the same result as the approach of backprojecting a $2 \mathrm{D}$ image described above. However, it is more time consuming, therefore the latter is preferred in this work.

It is worth noting that the elevation length of the reconstructed volume is equal to the acquired imaging depth and is not limited by the displacement distance. Thus out-of-plane absorbers can be visualized in an elevation distance of up to the acquired imaging depth as long as their signals are detected by the transducer array.

\subsubsection{D reconstruction based on back-projection and multiplication}

The number of displacement positions might be limited resulting in strong streak-like reconstruction artifacts as seen in Fig. 5.2(e). To reduce these artifacts, we use a reconstruction algorithm similar to the method proposed in [26, 27]. Here we call it back- 
projection and multiplication (BPM). In principle, all projections, before being summed up together, are combinatorially coupled and multiplied.

Let $b_{i}$ denote a 3D projection at position $i$ of the transducer array. $b_{i}$ is a 3D matrix. In direct back-projection (BP), reconstructed pressure distribution, $p_{B P}$ is the sum of all projections:

$$
p_{B P}=\sum_{i=1}^{M} b_{i},
$$

where $\mathrm{M}$ is the total number of projections, and in this case, it is the total number of displacement positions of the transducer array. In BPM, 2 projections $b_{i}$ and $b_{j}$ are coupled using signed geometric mean as [26]:

$$
\hat{b}_{i j}=\operatorname{sign}\left(b_{i} b_{j}\right) \sqrt{\left|b_{i} b_{j}\right|},
$$

where $b_{i} b_{j}$ is the element by element product of the two matrices $b_{i}$ and $b_{j}$. The reconstructed pressure distribution, $p_{B P M}$, in BPM is computed as [26]:

$$
p_{B P M}=\sum_{i=1}^{M-1} \sum_{j=i+1}^{M} \hat{b}_{i j} .
$$

$p_{B P M}$ is then band-pass filtered to remove the DC and high frequency components produced by multiplying the RF. In the end, $p_{B P M}$ is Hilbert transformed providing a 3D image.

Fig. 5.3 shows images in an xz plane using direct BP (Fig. 5.3(a)) and BPM (Fig. 5.3(b)). Streak-type reconstruction artifacts are observably reduced in BPM compared to BP. To provide more insight into reconstruction artifact reduction, we select signal, noise, and artifact windows as solid back, dotted white, and dashed white rectangles respectively, see in Fig. 5.3(a). The bigger artifact window covers a large area of reconstruction artifacts while the smaller one is on a specific reconstruction artifact. The yellow framed image at the bottom right corner of Fig. 5.3(a) is an enlargement of the absorber for a better visualization of the signal window. 

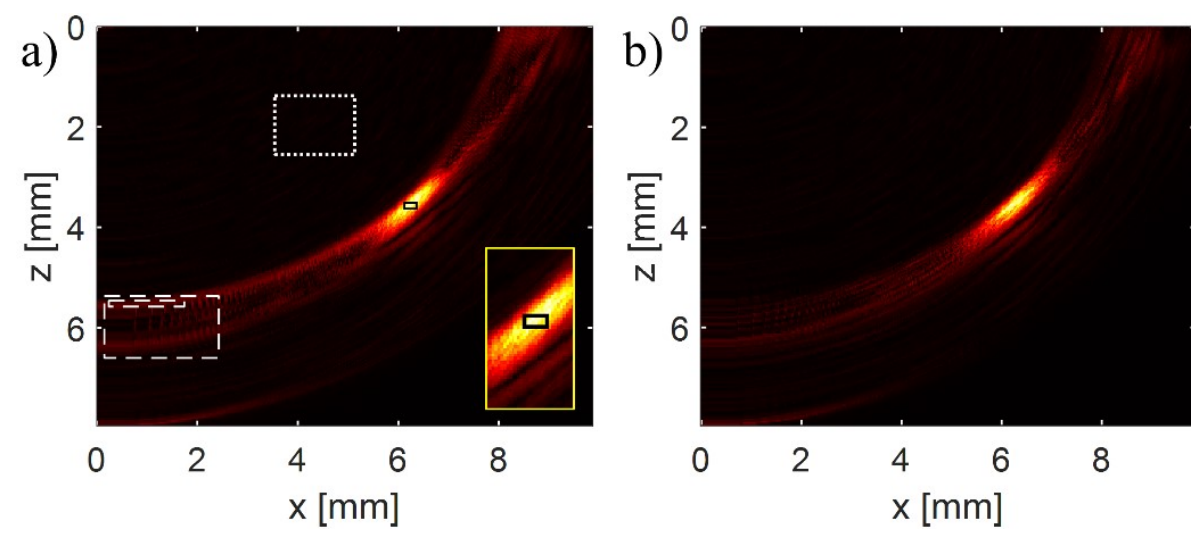

Fig. 5.3. Direct back-projection (a) vs back-projection and multiplication (b). Solid black, dotted white, and dashed white rectangles in (a) are signal, noise, and artifact windows respectively.

Let $\mathrm{S}, \mathrm{N}$, and $\mathrm{A}$ denote the mean value of all pixel values in the signal, noise, and artifact windows respectively. We then define signal-to-noise ratio (SNR) as S/N and artifact-tonoise ratio (ANR) as $\mathrm{A} / \mathrm{N}$. Table 5.1: Direct back-projection vs back-projection and multiplication. shows a comparison of SNR and ANR between direct BP and BPM. While SNR is approximately the same, ANR is significantly reduced in BPM. This is one of significant advantages of BPM over direct BP presented in [26, 27].

Table 5.1: Direct back-projection vs back-projection and multiplication.

\begin{tabular}{lcc}
\cline { 2 - 3 } & BP & BPM \\
\hline SNR & 68.53 & 68.04 \\
\hline ANR (large window) & 5.96 & 3.79 \\
\hline ANR (small window) & 9.06 & 2.74
\end{tabular}

We have just presented a 3D reconstruction algorithm based on BPM for axial displacement above. For elevation displacement, the reconstruction is in principle the same. At each elevation displacement position, a 3D projection is created. BPM is then used to reconstruct a $3 \mathrm{D}$ image. 


\subsection{Experimental setup}

Experiments were carried out using a handheld PAI probe, shown in Fig. 5.4. The probe is connected to a commercial US scanner MyLabOne (Esaote Europe BV, The Netherlands) which can acquire data at a sampling frequency of up to $50 \mathrm{MHz}$ with 12 bit digitization. It was used in research mode to acquire data for off-line processing in an external PC (Matlab 2019b, Intel Core i7 $3.41 \mathrm{GHz}, 8 \mathrm{~GB}$ of RAM).

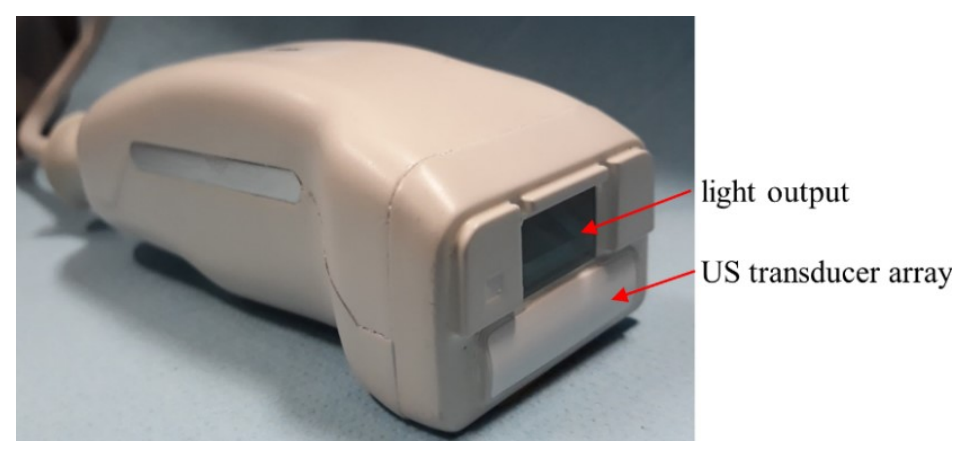

Fig. 5.4.. Integrated PAI probe.

In the probe, a US transducer array and a light source are integrated. The US transducer array comprises 128 elements with a pitch of $0.245 \mathrm{~mm}$. It has a center frequency of 7.5 $\mathrm{MHz}$ with a bandwidth of $100 \%$. The light source, integrated into the probe, consists of two stacks of diode lasers bars (10 bars per stack). It can emit light at 2 different wavelengths (760 and $940 \mathrm{~nm}$ ) at a repetition rate of up to $10 \mathrm{kHz}$. Table 5.2 presents specifications of the diode lasers working at a repetition rate of $0.5 \mathrm{kHz}$. As the $940 \mathrm{~nm}$ light has much higher pulse energy, we use light at this wavelength in our experiments.

Table 5.2. Lasers specifications at a repetition rate of $0.5 \mathrm{kHz}$.

\begin{tabular}{llll}
\hline wavelength $[\mathrm{nm}]$ & $\begin{array}{l}\text { pulse energy at } \\
\text { the output }[\mathrm{mJ}]\end{array}$ & pulse width $[\mathrm{ns}]$ & $\begin{array}{l}\text { Fluence at the output } \\
\left(1 / \mathrm{e}^{2}\right)\left[\mathrm{mJ} / \mathrm{cm}^{2}\right]\end{array}$ \\
\hline 760 & 0.58 & 63.6 & 0.74 \\
\hline 940 & 1.66 & 53.4 & 2.01 \\
\hline
\end{tabular}

The probe is mounted on 2 motorized translation stages (MTS50A-Z8, Thorlabs, Germany). These stages are used to displace the probe axially and elevationally. 


\subsection{Results}

A total of 11 pressure distribution images at 11 displacement positions were acquired: 1 at the original position, 5 for axial displacements and 5 for elevation displacements. The displacement step size was $0.525 \mathrm{~mm}$ resulting in total $2.625 \mathrm{~mm}$ displacement axially and elevationally. Acquired raw data was applied a 5th-order high-pass Butterworth filter with a cut-off frequency of $0.5 \mathrm{MHz}$ and averaged over 100 pulses. We then reconstructed pressure distribution images using a Fourier transform based reconstruction algorithm [25].

We first determined axial, lateral, and elevation resolutions of 3D images and compared the resolutions between axial and elevation displacement. We then demonstrated the reconstruction algorithm with in vivo experiments. We also measured the sensitivity of the transducer array which afterwards we used to improve our 3D images.

In all supplementary videos presenting 3D reconstruction, we added an orange frame into the $3 \mathrm{D}$ images to represent the imaging plane.

\subsubsection{Resolutions of an in-plane image feature}

Fig. 5.5(a) shows the probe with an associated coordinate system. Fig. 5.5(b) illustrates the experiment configuration from a side view of the probe. An absorber cut out from a black suture (USP 5/0, Vetsuture Nylon, The Netherlands) with a thickness of $\sim 140 \mu \mathrm{m}$ (smaller than the imaging resolutions of this probe) was used in this experiment as an acoustically point-like absorber. This absorber was embedded in agarose (2\%) in a petri dish (Greiner Bio-One GmbH, Germany) and placed underneath the transducer array inside the imaging plane, see Fig. 5.5(b)). The coupling medium was demi-water.
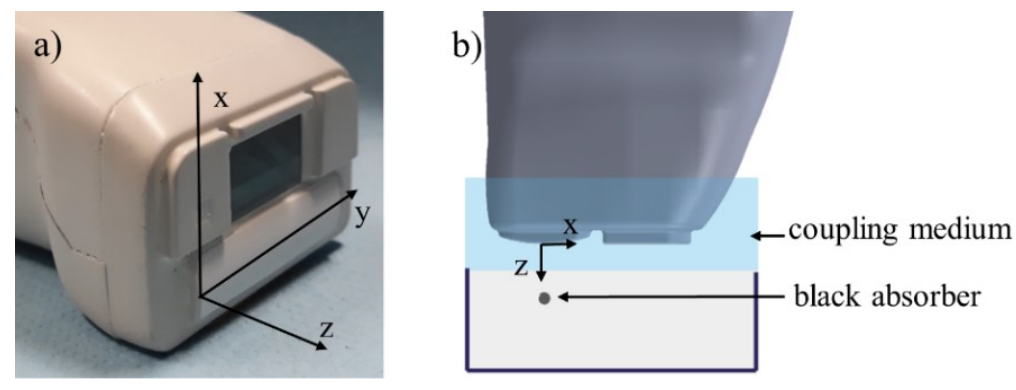

Fig. 5.5. In-plane absorber configuration. (a) PAI probe with an associated coordinate system. (b) Schematic elevation view of the experiment configuration. 
Fig. 5.6 shows results of 3D reconstruction using axial displacement (Fig. 5.6(a)-(b)) (see also Appendix 2.7) and elevation displacement (Fig. 5.6(c)-(d)) (see also Appendix 2.8). Columns from left to right are in-plane images and in an xz plane crossing the absorber, respectively. Images in the same row share the same color bar.

The SNR in the reconstruction using elevation displacement is considerably lower than using axial displacement. Consequently, electrical noise at the depth of $\sim 2 \mathrm{~mm}$ is more pronounced in the reconstructed image. The reason is that in elevation displacement, inplane absorbers relatively move away from the imaging plane and the sensitivity of the transducer decreases further outside of the imaging plane.
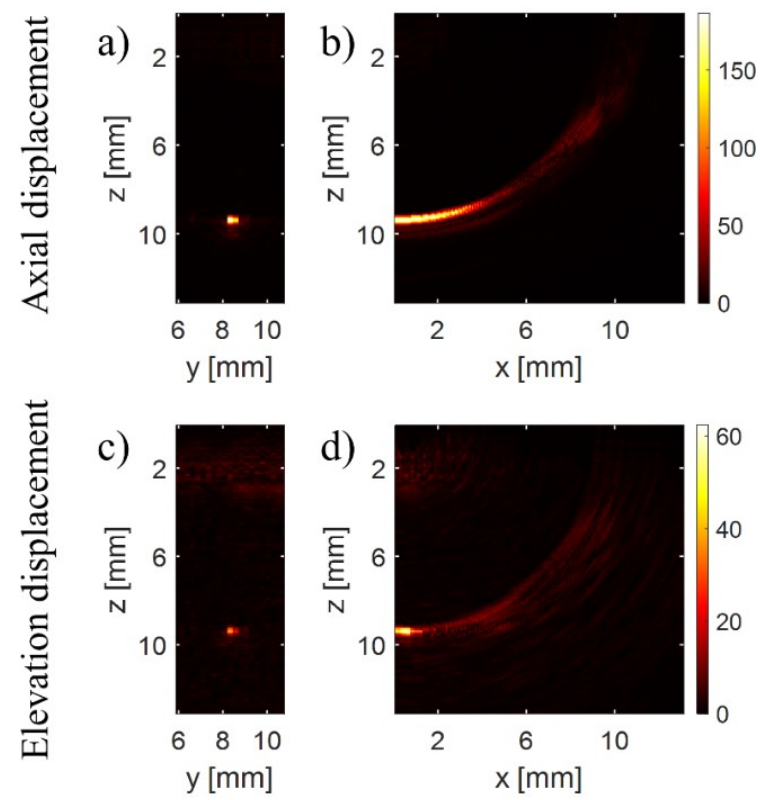

Fig. 5.6. Reconstructed images using axial ((a)-(b)) and elevation ((c)-(d)) displacement. (a) and (c) In-plane images. (b) and (d) Images in an xz plane crossing the absorber.

3D reconstruction using axial displacement does not provide elevation information of in-plane absorbers. The reason is that the imaging plane does not change elevationally. It can be seen in Fig. 5.6(c) that the point-like source is reconstructed as a curve elevationally. However, this is not the case in using elevation displacement despite of strong effect of streak-type reconstruction artifacts. 
Fig. 5.7 shows elevation, lateral, and axial profiles (top to bottom) of the reconstructed image feature using axial, and elevation displacement (left to right). All figures share the same y label shown on the left. The profiles are fitted into Gaussian curves. Resolutions are then determined as the FWHM of the fitted Gaussian curves, shown in Table 5.3.
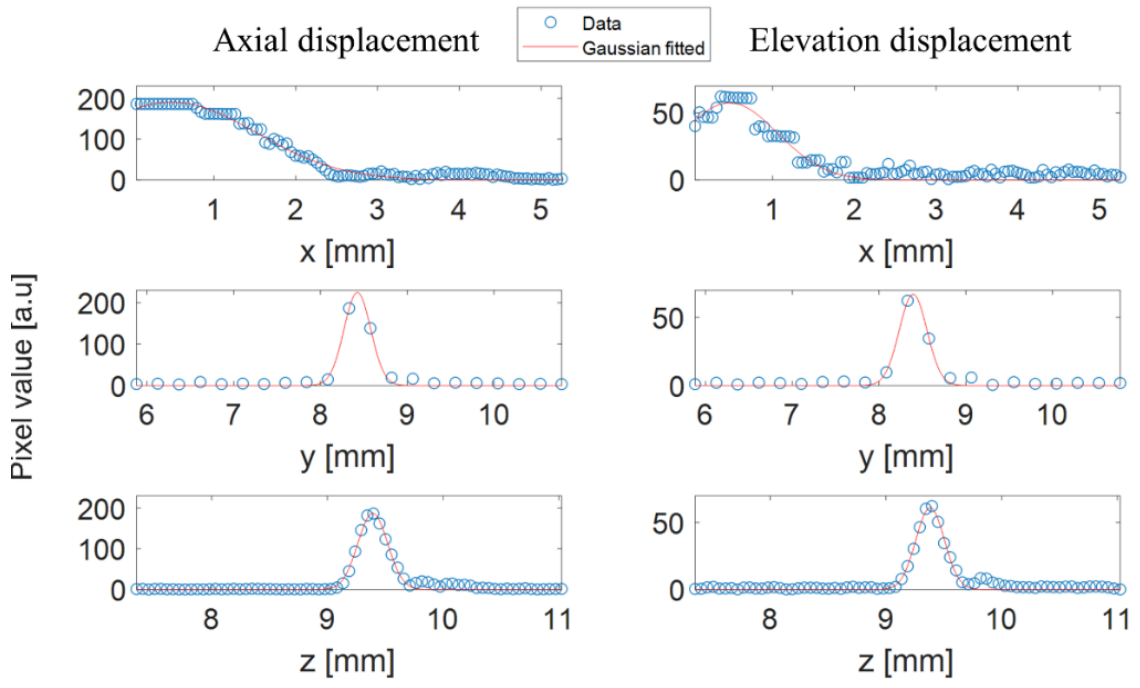

Fig. 5.7. Profiles of the reconstructed image features using axial (left column) and elevation (right column) displacement. From top to bottom: elevation, lateral, and axial profiles.

The lateral and axial resolutions are approximately the same in the reconstruction using axial and elevation displacement while the elevation resolution is better (almost 2 times) with using elevation displacement.

Table 5.3. Resolutions of an in-plane image feature.

Axial displacement [mm] $\quad$ Elevation displacement [mm]

\begin{tabular}{lcc}
\hline $\mathrm{r}_{\text {elevation }}$ & 2.43 & 1.38 \\
\hline $\mathrm{r}_{\text {lateral }}$ & 0.36 & 0.38 \\
\hline $\mathrm{r}_{\text {axial }}$ & 0.30 & 0.29 \\
\hline
\end{tabular}




\subsubsection{Resolutions of an out-of-plane artifact}

The same experimental configuration as described in section 5.4.1 was used. The black absorber in this case was placed $\sim 4 \mathrm{~mm}$ axially and $\sim 4.5 \mathrm{~mm}$ elevationally away from the transducer array, see Fig. 5.8(a). Fig. 5.8(b)-(e) are results of 3D reconstruction using axial ((b)-(c)) (see also Appendix 2.9) and elevation ((d)-(e)) (see also Appendix 2.10) displacement. Images in the same row share the same color bar on the right. Fig. 5.8(b) and (d) and Fig. 5.8(c) and (e) show images in an yz and an xz plane crossing the absorber respectively.
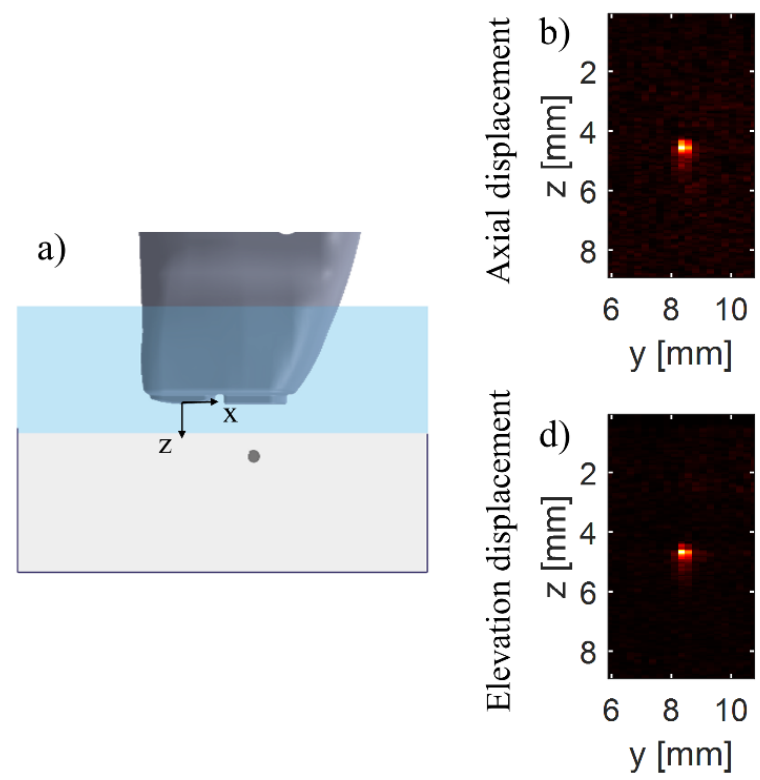
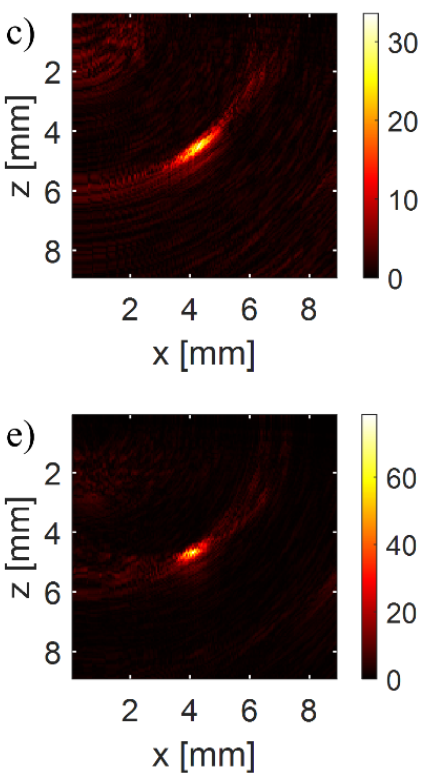

Fig. 5.8. Out-of-plane absorber imaging. (a) Schematic elevation view of the experiment configuration. (b)-(c) and (d)-(e) are reconstructed images using axial and elevation displacement respectively. (b) and (d) Images in an yz plane crossing the absorber. (c) and (e) Images in an xz plane crossing the absorber.

Table 5.4 presents the resolutions of an out-of-plane artifact in 3D reconstruction using axial and elevation displacement. The axial and lateral resolutions are slightly better with using elevation displacement. This is because in elevation displacement, out-of-plane absorbers relatively move towards the imaging plane. The elevation resolution, on the other hand, is approximately the same. 
Table 5.4. Resolutions of an out-of-plane artifact.

\begin{tabular}{ccc} 
& Axial displacement $[\mathrm{mm}]$ & Elevation displacement $[\mathrm{mm}]$ \\
\hline $\mathrm{r}_{\text {elevation }}$ & 0.53 & 0.54 \\
\hline $\mathrm{r}_{\text {lateral }}$ & 0.39 & 0.36 \\
\hline $\mathrm{r}_{\text {axial }}$ & 0.40 & 0.32
\end{tabular}

It is worth noting that the resolutions also depend on the location of the absorber. However, this study of in-plane and out-of-plane absorber imaging provides some insight into comparison between reconstruction using axial and elevation displacement.

\subsubsection{In vivo experiment}

We also demonstrated the reconstruction using axial displacement with in vivo experiments. We compared the results with the reconstruction using elevation displacement. Fig. 5.9. In vivo experiment. (a) Experiment configuration. (b) Acquired PA image. Dashed circle indicates OPAs. shows the configuration of the in vivo experiments. We imaged a volunteer's finger with a black ink mark on the skin mimicking a strongly absorbing epidermis or objects like birth marks as an out-of-plane absorber. The finger was positioned in such a way that the ink mark was a few millimeters away from the transducer array elevationally but still inside the light beam, see Fig. 5.9(a). Demi-water was used as a coupling medium. Fig. 5.9(b) shows an acquired PA image. Features (dashed white circle) move up along with the axial displacement (seen in Appendix 2.11) revealing them as OPAs. They appear at the position that should be expected from the elevational location of the ink mark. Probably, these are OPAs of the ink mark. 

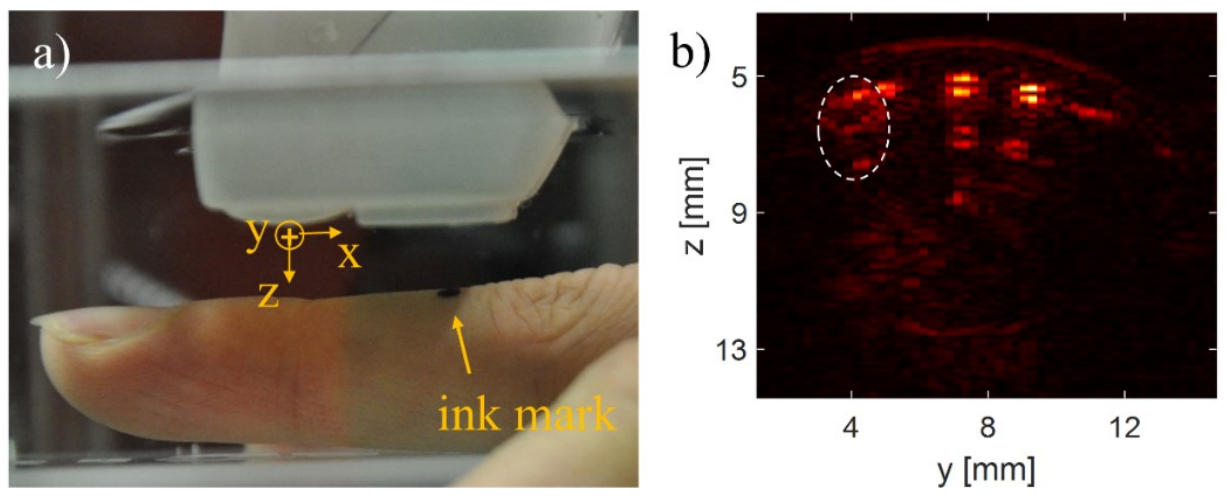

Fig. 5.9. In vivo experiment. (a) Experiment configuration. (b) Acquired PA image.

Dashed circle indicates OPAs.

At a certain distance of the axial displacement and beyond, OPAs disappear, seen in Appendix 2.7. The reason is that at that distance, the ink mark was outside of the light beam. Furthermore, few features move down during the displacement. These are probably reflections caused by signals generated due to the absorption by the transducer array and reflected back off the skin and bone layers. These reflections move down since the distance of the probe to the reflectors increases during the displacement.

Fig. 5.10 shows volume renderings of 3D reconstruction using axial (Fig. 5.10(a)-(c)) (see also Appendix 2.12) and elevation (Fig. 5.10(d)-(f)) (see also Appendix 2.13) displacement. For a better visualization, 3D images are thresholded one sixth of each image's maximum. Images in the same row share the same color bar on the right. The $3 \mathrm{D}$ images are presented in different view angles (in different columns). The solid black arrow in all images indicates the ink mark. It is $\sim 4 \mathrm{~mm}$ away from the imaging plane. 

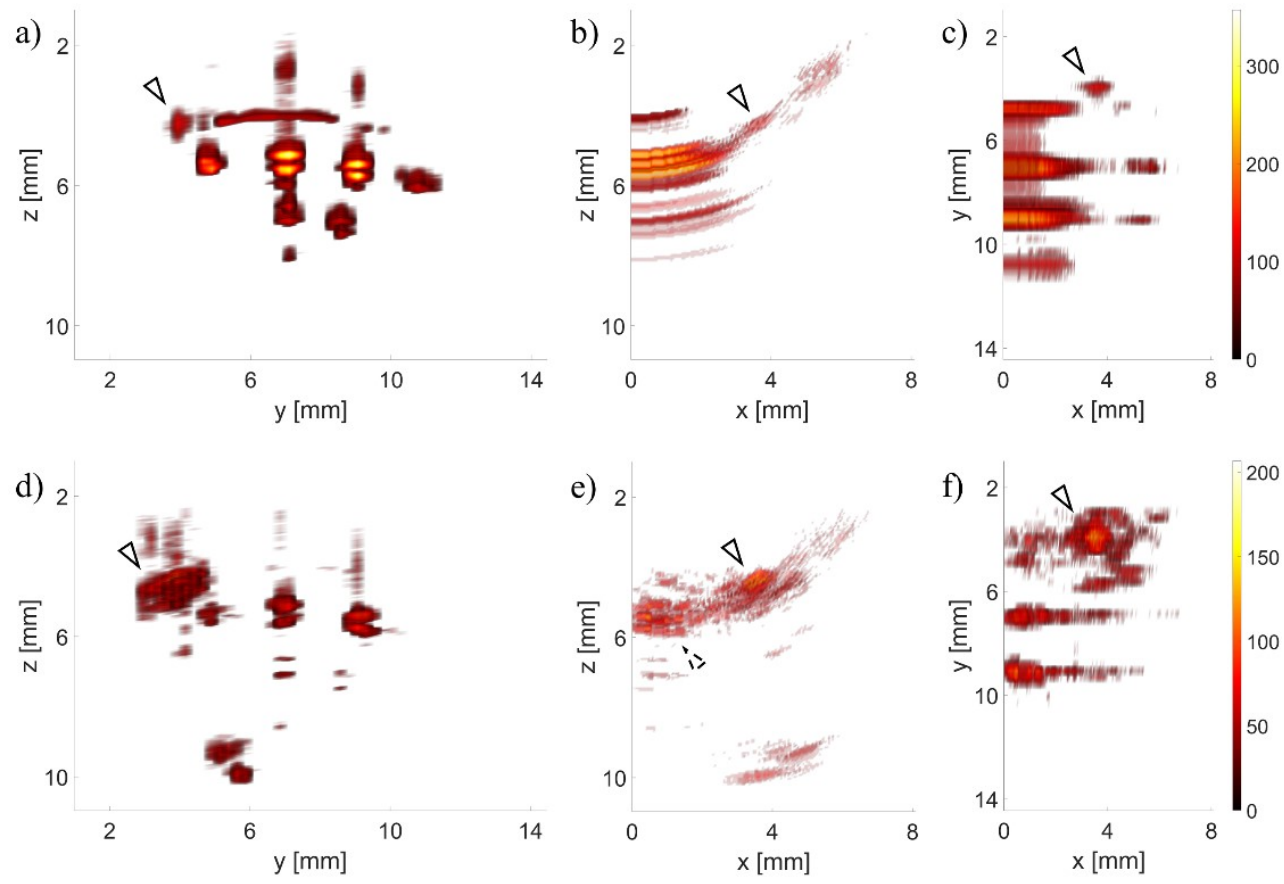

Fig. 5.10. In vivo 3D reconstruction using axial displacement ((a)-(c)) and elevation displacement ((d)-(f)). Solid black arrows indicate the ink mark. Dashed black arrow indicates some blood vessels.

Only some blood vessels can be visualized after thresholding in the case of using elevation displacement. This is due to the SNR of the in-plane image being lower in the reconstruction using elevation displacement than using axial displacement, as pointed out in section 5.4.1. As also mentioned in section 5.4.1, reconstruction using axial displacement does not provide elevation information of in-plane absorbers. This can be seen again in Fig. $5.10 \mathrm{~b}$ ) in which skin and blood vessels appear as curves elevationally. In contrast, the blood vessels appear as thread structures elevationally (dashed arrow, Fig. 5.10(e)) in reconstruction using elevation displacement.

\subsubsection{Out-of-plane artifact reduction}

In addition to providing a 3D view of out-of-plane absorbers, the $3 \mathrm{D}$ reconstruction using axial transducer array displacement also significantly reduces OPAs in the in-plane images. In this way, segmentation is not needed as in the method used in the previous work [18]. In this section, we will further analyze the experimental results presented in sections 
5.4.2 and 5.4.3 to give more insight into OPA reduction, and compare with the previous method using segmentation.

Fig. 5.11 shows images of the out-of-plane absorber phantom (upper row) and in vivo experiment (lower row) presented in section 5.4.2 and 5.4.3 respectively. All images in the same row share the same color bar shown at the end of each row. For a better visualization, images of the out-of-plane absorber phantom are linearly scaled to have the same dynamic range while images of the in vivo experiment are in their own dynamic range. Images in different columns in order from left to right are acquired images, in-plane images of the 3D reconstruction using axial transducer array displacement, and OPA removed images using the previous method [18] (axial transducer array displacement with segmentation). Solid and dashed rectangles represent OPA and noise windows respectively.
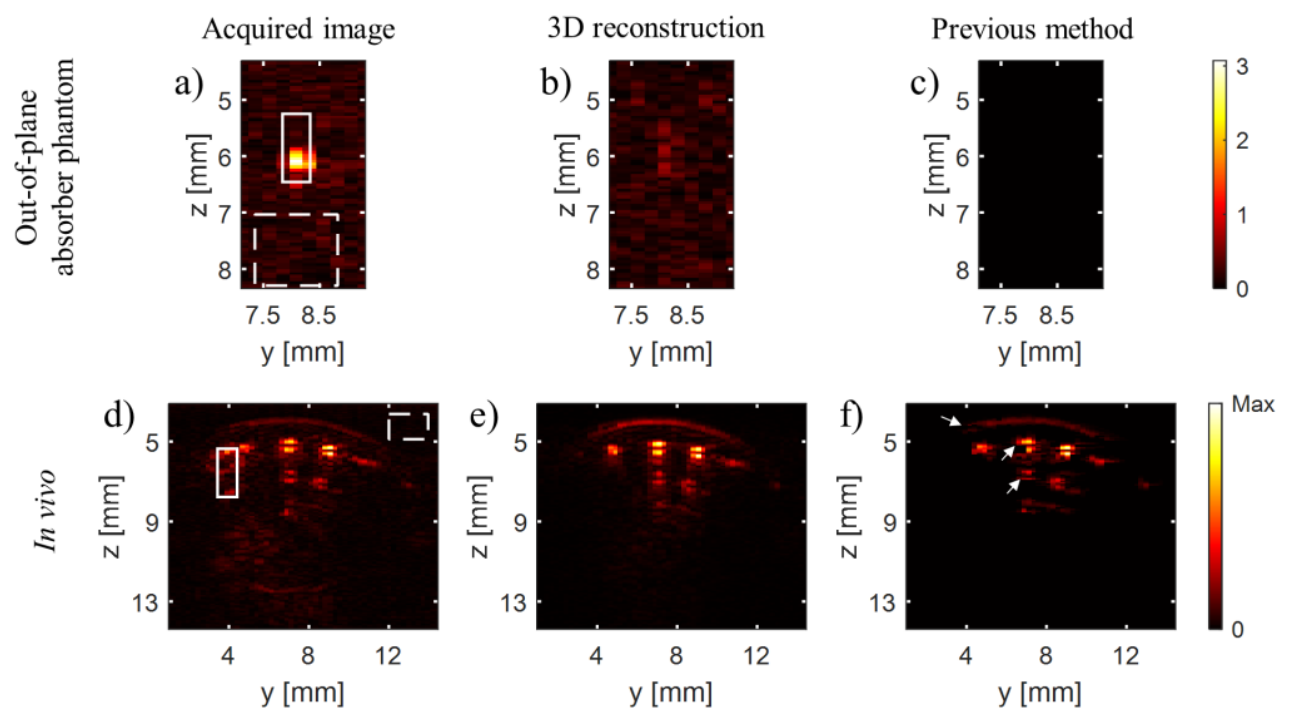

Fig. 5.11. OPA reduction. Upper row: images of the out-of-plane absorber phantom presented in section 5.4.2. Lower row: images of the in vivo experiment presented in section 5.4.3. Left column: original acquired images. Middle column: in-plane images of the 3D reconstruction using axial displacement. Right column: OPA removed images using the previous method [18]. Solid and dashed rectangles represent OPA and noise windows respectively. Arrows indicate removed image features.

As OPAs move up during the axial displacement, they are averaged out in the in-plane image of the 3D reconstruction, seen in Fig. 5.11(b) and (e), whereas they are removed by 
setting the corresponding pixel value to 0 using the previous method, seen in Fig. 5.11(c) and (f). The white arrows in Fig. 5.11(f) show in-plane image features that are partly removed.

Table 5.5 presents an ANR comparison between acquired PA images and in-plane images of the 3D reconstruction using axial transducer array displacement. As the pixel value of the OPAs is set to 0 in the images of using the previous method, so they are not included in this ANR analysis.

Table 5.5. ANR comparison between acquired $P A$ images and in-plane images of the 3D reconstruction using axial transducer array displacement.

\begin{tabular}{lll}
\cline { 2 - 3 } & Acquired image & 3D reconstruction \\
\hline Phantom & 3.23 & 1.60 \\
\hline In vivo & 6.35 & 4.15 \\
\hline
\end{tabular}

It can be seen that OPAs are clearly reduced in the image of the 3D reconstruction using axial transducer array displacement. The reflections of the transducer array noted in section 5.4.3 are also averaged out as they move down during the axial displacement, seen in Fig. 5.11(e). These artifacts (the OPAs and the reflections) are completely removed in the images of using the previous method. Some image features (marked with white arrows) are also removed due to some slight movement during the experiment, seen in Fig. 5.11(f).

\subsection{5. $\quad x z$ sensitivity compensation}

Compensating the sensitivity of the transducer array might improve the reliability of the reconstructed image. The sensitivity compensation can be achieved by weighting the backprojected signals [28]. In this work, the out-of-plane sensitivity of the transducer array is much lower than the in-plane sensitivity. Consequently, the reconstructed initial pressure of out-of-plane absorbers is much lower than its real initial pressure. We experimentally measure the sensitivity of the transducer array and take it into account in the 3D reconstruction.

Fig. 5.12(a) illustrates the configuration of measuring the sensitivity of the transducer array. A US probe which consists of only a transducer array was used. The transducer array is identical to the one in the PAI probe. The US probe was mounted on 2 translation stages 
which could translate axially and elevationally. The sample was a black absorber embedded in agarose as described in section 5.4.1. The light source (at a wavelength of $790 \mathrm{~nm}$ ), described in [18], and sample were fixated. The coupling medium was a suspension of $1 \%$ Intralipid $20 \%$ in demi-water.

We translated the probe as a raster scan in the xz plane. At each position of the probe, one image was acquired with averaging of 100 laser pulses. In such a way, we could neglect the laser fluctuation and assume that the initial pressure from the absorber was a constant. The maximum pixel value of the absorber in each image was extracted as a sensitivity value at the corresponding position of the probe. Fig. 5.12(b) shows a sensitivity map extracted from the whole scan. The $0 \mathrm{~mm}$ on the $\mathrm{x}$ axis presents the position when the absorber was exactly underneath the probe.

The step size of the raster scan was larger than the spatial size of a pixel in 3D reconstruction. We use interpolation to match the spatial pixel size of the sensitivity map with reconstructed images.
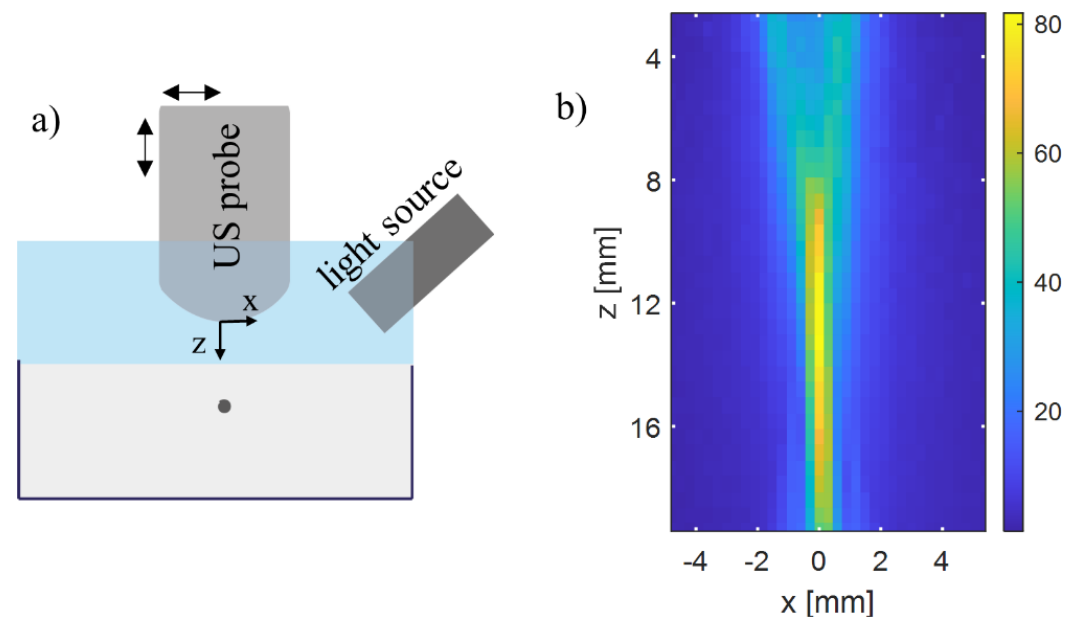

Fig. 5.12. Measuring the spatial sensitivity distribution of the transducer array. (a) Schematic experiment setup. (b) xz sensitivity.

We assume that all transducer elements have the same xz sensitivity map. Therefore, we apply the $\mathrm{xz}$ sensitivity compensation map equally laterally to the reconstructed $3 \mathrm{D}$ image. This point will be discussed further in the Discussion section. Additionally, the sensitivity of the transducer array also depends on the acoustic wavelength of the source. This, however, 94 
was not considered in the sensitivity compensation. We will also discuss further this point in the Discussion section.

In $3 \mathrm{D}$ reconstruction using axial or elevation displacement, the sensitivity of the transducer array is also shifted corresponding to the displacement. We thus shift the sensitivity map to match the displacement and expand it laterally to a 3D sensitivity map. We then divide (element by element division) the corresponding 3D projection by the 3D sensitivity map. All sensitivity compensated 3D projections are then used for reconstruction using BPM.

\subsubsection{Phantom validation}

We first validate the sensitivity compensation with a phantom experiment. The experiment configuration is shown in Fig. 5.13(a). It is similar to the configuration described in section 5.4.1. There is one absorber placed inside the imaging plane and another $\sim 3 \mathrm{~mm}$ outside of the imaging plane. Fig. 5.13(b) and (d) are volume renderings of 3D reconstruction using axial and elevation displacement (see also Appendix 2.14 and Appendix 2.15) respectively. These 3D images are thresholded in such a way to visualize the two absorbers. The arrow in Fig. 5.13(b) indicates a reconstruction artifact while most of the reconstruction artifacts are thresholded out in the reconstruction using elevation displacement. Sensitivity compensation is then applied to these images resulting in images on the right (Fig. 5.13(c) and (e)) (see also Appendix 2.16 and Appendix 2.17). All images are displayed in their own dynamic range with a color bar on the top.

With sensitivity compensation, the signal of the out-of-plane absorber is considerably enhanced. This is reasonable since the out-of-plane absorber was placed closer to the light output and to the center of the laser beam compared to the in-plane absorber.

Reconstruction artifacts that are elevationally far away from the imaging plane are strongly enhanced with the sensitivity compensation. As a result, they are even more pronounced than real image features. A reconstruction artifact marked with an arrow in Fig. 5.13(b) is an example. This artifact is significantly enhanced with the transducer sensitivity taken into account, seen in Fig. 5.13(c). 


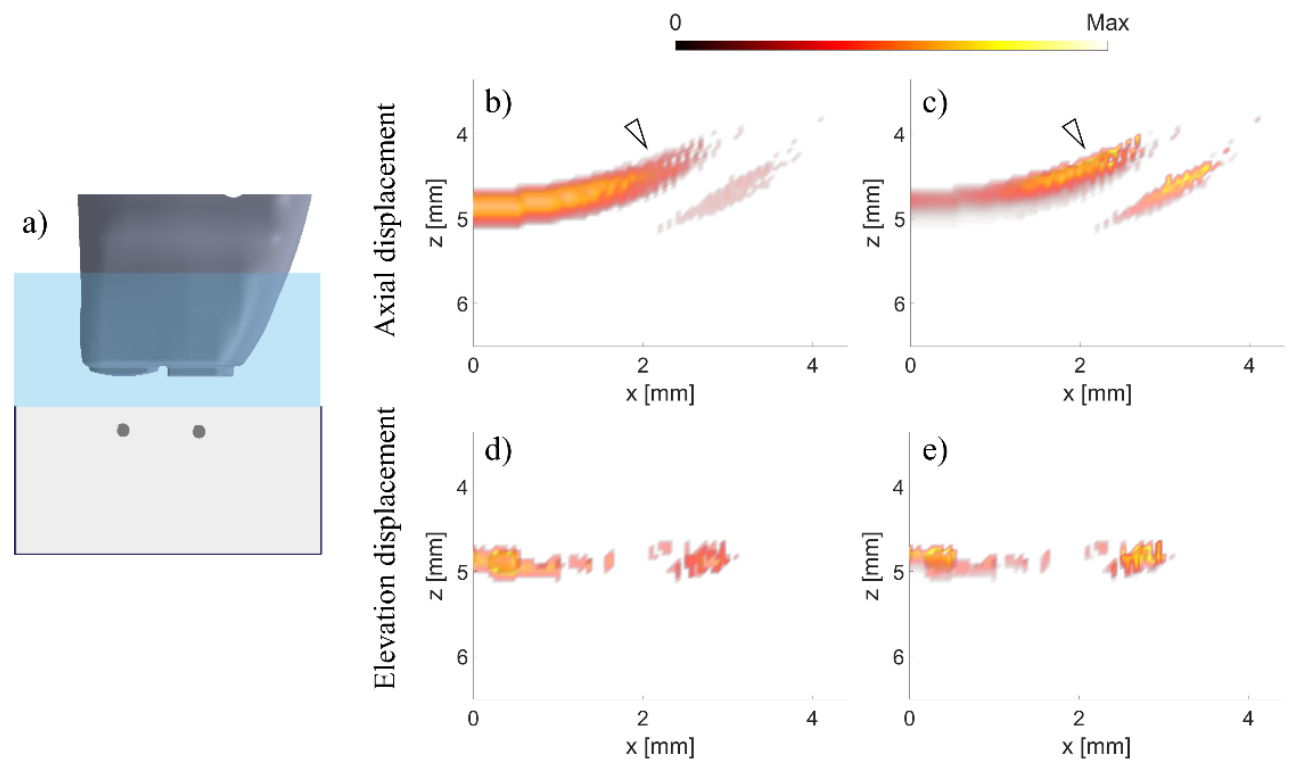

Fig. 5.13. In-plane and out-of-plane absorber imaging. (a) Experiment configuration. 3D images using axial displacement without (b) and with (c) sensitivity compensation respectively. 3D images using elevation displacement without (d) and with (e) sensitivity compensation. Arrows indicate a strongly enhanced reconstruction artifact.

\subsubsection{In vivo validation}

Fig. 5.14 shows results of the in vivo experiment with sensitivity compensation. From top to bottom are images without and with sensitivity compensation alternatively row by row. The first 2 rows and the last 2 rows are results of reconstruction using axial displacement and elevation displacement respectively. Images in the same row have the same dynamic range and each row has its own dynamic range. Thus the color bar has a dynamic maximum. The solid arrow in all images indicates the ink mark.

In the images with sensitivity compensation, the signal of the ink mark is considerably stronger than the signal from the skin and blood vessels. This is reasonable since the ink mark was positioned closer to the light output resulting in higher local light fluence. In addition, the ink mark is more absorbing than the skin and blood vessels.

The sensitivity map was measured from $\sim 2.5 \mathrm{~mm}$ on the $\mathrm{z}$ axis and up to $\sim 5.3 \mathrm{~mm}$ on the $\mathrm{x}$ axis. This is the reason why sensitivity compensated images appear as cropped at those boundaries. 
The dashed arrow in Fig. 5.14(b) indicates a reconstruction artifact which is significantly enhanced with the transducer sensitivity taken into account, seen in Fig. 5.14(e). Similarly, Fig. 5.14(h) and (k) present another example in the case of using elevation displacement. 

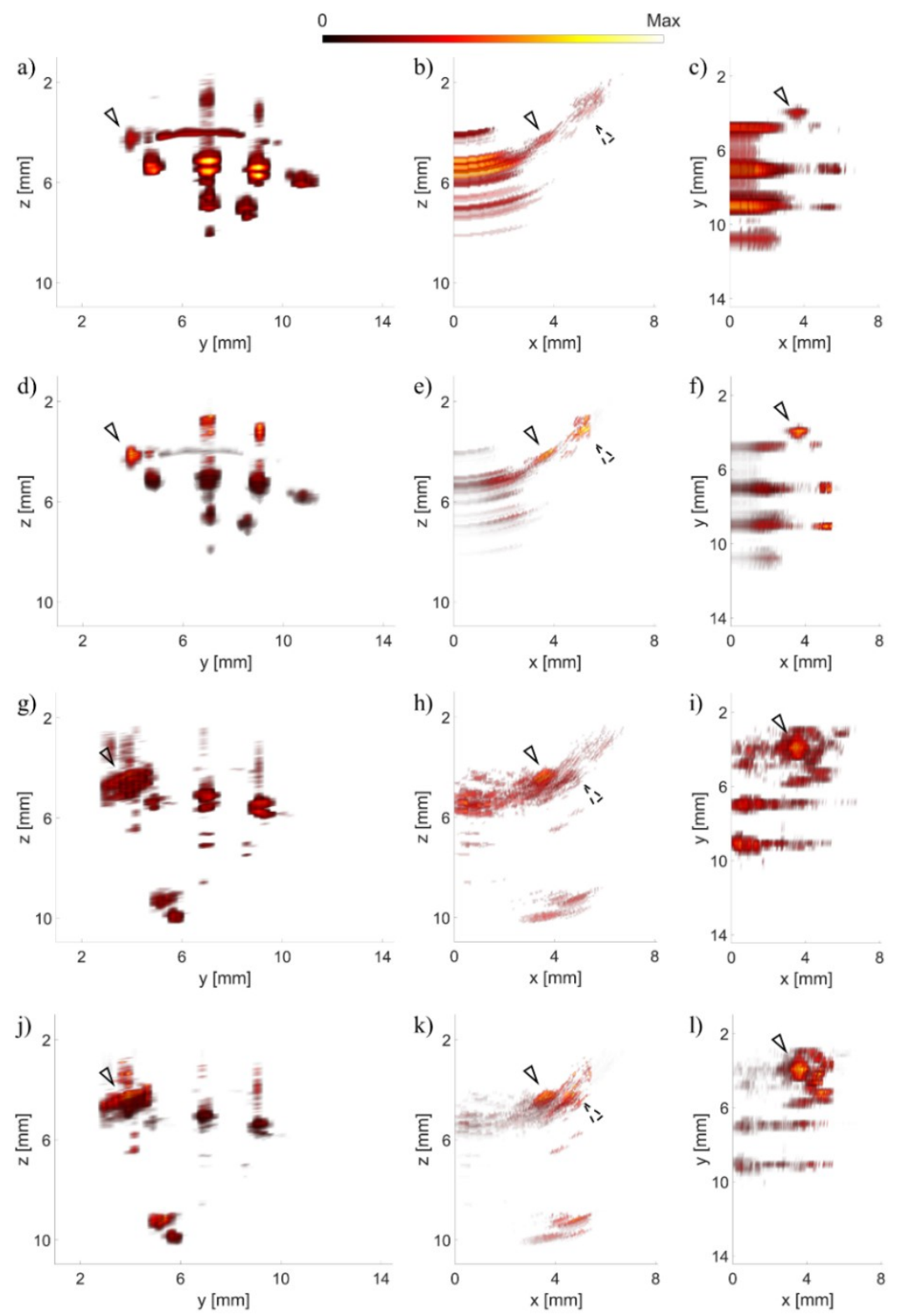

Fig. 5.14. In vivo 3D reconstruction with sensitivity compensation. 3D images using axial displacement without ((a)-(c)) and with ((d)-(f)) (see also Appendix 2.18) sensitivity compensation. 3D images using elevation displacement without ((g)-(i)) and with ((j)-(1)) (see also Appendix 2.19) sensitivity compensation. Solid arrows indicate the ink mark, dashed arrows indicate strongly enhanced reconstruction artifacts. 


\subsection{Discussion}

The occurrence of OPAs negatively influences the quality and reliability of the images in PAI. Correcting OPAs thus benefits the translating of PAI to clinics. In addition to the capability of identifying and removing OPAs, our proposed method provides a 3D view of out-of-plane absorbers. Moreover, it offers significant advantages over existing 3D imaging methods in terms of visualizing out-of-plane absorbers. First, for a given measurement time, the proposed method enhances the quality of the in-plane images since the probe remains in the imaging planes and adds up all in-plane images while this is not the case with elevation displacement. Secondly, the elevation visualization is not limited by the displacing distance as in the case of 3D reconstruction by scanning a linear transducer array [22, 23]. The proposed method can spatially visualize in a larger extent than the displacing distance, up to the acquired imaging depth. Thirdly, the method does not require a 2D transducer array for the $3 \mathrm{D}$ reconstruction as in $[20,21] .2 \mathrm{D}$ transducer arrays require complex associated systems resulting in unaffordable cost for many clinical applications. Lastly, we demonstrated the method using a linear-transducer-array probe with integrated lasers showing its promising potential for clinical use.

In the proposed 3D reconstruction method, OPAs and reflections of the transducer array are averaged out in the in-plane image as shown in section 5.4.3. In this way, background information behind the OPAs still remains whereas it is removed along with OPAs in our previous work [18]. Additionally, reducing OPAs with the proposed method does not rely on segmentation, and is more robust to slight movement of the sample than in the case of the previous method.

Though the 3D reconstruction using axial displacement can three-dimensionally visualize out-of-plane absorbers, it cannot show in-plane absorbers in an elevationally correct manner. All in-plane absorbers appear in a curve shape elevationally. This is the main drawback of this method. However, our main scope is to correct in-plane images and visualize out-of-plane absorbers.

Unlike in our previous work [18], the light source is also displaced in this work since it is integrated in the probe, resulting in changes of the illumination during the displacement. However, since the displacement was just a few millimeters, we did not observe significant 
influence of the illumination changes on our reconstructed image. On the other hand, validating our proposed method with a compact and low-cost PAI probe shows its potential for clinical application.

A limited number of displacement positions might lead to strong streak-type reconstruction artifacts. In this work, we used back-projection and multiplication (BPM) which in principle is similar to delay-multiply-and-sum [26, 27] to improve the reconstruction. Applying methods to further reduce the reconstruction artifacts, such as [29, 30], will be included in our future work.

BPM improves the reconstruction at a cost of longer processing time due to a greater amount of calculation [26]. The amount of calculation is proportional to $M$ (number of projections) in direct $\mathrm{BP}$, and $\mathrm{M}(\mathrm{M}-1) / 2$ in BPM . In this work, for a ROI of $18 \times 16 \times 18$ $\mathrm{mm}^{3}(\mathrm{x} \times \mathrm{y} \times \mathrm{z})$ it took $\sim 5$ seconds for each reconstruction run. In this amount of processing time, creating $63 \mathrm{D}$ projections took $\sim 0.48(6 \times 0.08)$ seconds while BPM took $\sim 4.5$ seconds. Using a faster reconstruction algorithm [31] would significantly reduce the reconstruction time.

In our previous work [18, 19], it took 2-3 minutes for each displacement experiment due to low repetition rate of the laser $(20 \mathrm{~Hz})$ and slow translation stages. In this work, the experiment time was reduced to a few tens of seconds thanks to the high repetition rate of the integrated laser. However, this is still far from real-time imaging. The main part the experiment time was due to the low speed of the translation stages. In addition, we did not use the highest repetition rate of the laser, $10 \mathrm{kHz}$ ( $1 \mathrm{kHz}$ was used). Using faster translation stages and a higher repetition rate of the laser might significantly reduce further the acquisition time.

As pointed out in [18], the principle of identifying and removing OPAs also holds for lateral out-of-plane absorbers. OPAs of lateral out-of-plane absorbers still move along the axial displacement of the probe. Thus visualizing lateral out-of-plane absorbers is also feasible.

As shown in sections 5.4.1 and 5.4.2, elevationally displacing the probe can also form a $3 \mathrm{D}$ view of out-of-plane absorbers with more elevation information and a better elevation resolution of in-plane image features compared to the proposed method. However as a cost, 
for a given total measurement time the quality of the in-plane image is significantly worse, shown in sections 5.4.1 and 5.4.3. Therefore, the proposed method is more favorable in scope of correcting OPAs in the in-plane image.

In sensitivity compensated reconstruction, we used a sensitivity map measured on an acoustically point-like source for other samples. This is not an ideal way for compensating the sensitivity since the sensitivity of the transducer also depends on the acoustic wavelength of the source. In PAI, especially in in vivo imaging, the acoustic wavelength of the signal might be unknown leading to erroneous compensation for the sensitivity. On the other hand, we also applied a measured sensitivity map to all transducer elements assuming they are all the same. This might be incorrect due to interferences between transducer elements, hence we did not include the influence of the sensitivity compensation on artifact reduction. Applying a correct sensitivity map of the transducer array will make the sensitivity compensation more proper.

\subsection{Conclusion}

We have demonstrated a three-dimensional (3D) reconstruction approach by using axial transducer array displacement. This is a new advantage of our recently proposed method [18, 19] for identifying and removing out-of-plane artifacts (OPAs). By displacing the transducer array axially for a few millimeters only, we could three-dimensionally visualize out-of-plane absorbers within an elevation distance of up to the acquired imaging depth and also reduce OPAs in the in-plane image. We also compared the results with the $3 \mathrm{D}$ reconstruction using elevation displacement. We then improved the reconstruction by taking the sensitivity of the transducer array into account. In vivo demonstrations using a compact photoacoustic imaging system show promising clinical applicability of our proposed method. 


\subsection{References}

[1] I. Steinberg, D. M. Huland, O. Vermesh, H. E. Frostig, W. S. Tummers, and S. S. Gambhir, "Photoacoustic clinical imaging," Photoacoustics, (2019).

[2] K. S. Valluru, K. E. Wilson, and J. K. Willmann, "Photoacoustic Imaging in oncology: translational preclinical and early clinical experience," Radiology 280(2), 332-349 (2016).

[3] S. Manohar, and M. Dantuma, "Current and Future Trends in Photoacoustic Breast Imaging," Photoacoustics, (2019).

[4] M. Heijblom, D. Piras, F. M. van den Engh, M. van der Schaaf, J. M. Klaase, W. Steenbergen, and S. Manohar, "The state of the art in breast imaging using the Twente Photoacoustic Mammoscope: results from 31 measurements on malignancies," European radiology 26(11), 3874-3887 (2016).

[5] S. M. Schoustra, D. Piras, R. Huijink, T. J. op't Root, L. Alink, W. M. Kobold, W. Steenbergen, and S. Manohar, "Twente Photoacoustic Mammoscope 2: system overview and three-dimensional vascular network images in healthy breasts," Journal of Biomedical Optics 24(12), 121909 (2019).

[6] P. J. van den Berg, K. Daoudi, H. J. B. Moens, and W. Steenbergen, "Feasibility of photoacoustic/ultrasound imaging of synovitis in finger joints using a point-of-care system," Photoacoustics 8, 8-14 (2017).

[7] J. Jo, G. Xu, M. Cao, A. Marquardt, S. Francis, G. Gandikota, and X. Wang, "A Functional Study of Human Inflammatory Arthritis Using Photoacoustic Imaging," Scientific reports 7(1), 15026 (2017).

[8] M. Toi, Y. Asao, Y. Matsumoto, H. Sekiguchi, A. Yoshikawa, M. Takada, M. Kataoka, T. Endo, N. Kawaguchi-Sakita, and M. Kawashima, "Visualization of tumor-related blood vessels in human breast by photoacoustic imaging system with a hemispherical detector array," Scientific Reports 7, (2017). 
[9] Q. Yao, Y. Ding, G. Liu, and L. Zeng, "Low-cost photoacoustic imaging systems based on laser diode and light-emitting diode excitation," Journal of Innovative Optical Health Sciences 10(04), 1730003 (2017).

[10] M. K. A. Singh, W. Steenbergen, and S. Manohar, "Handheld probe-based dual mode ultrasound/photoacoustics for biomedical imaging," in Frontiers in Biophotonics for Translational Medicine(Springer, 2016), pp. 209-247.

[11] K. Daoudi, P. Van Den Berg, O. Rabot, A. Kohl, S. Tisserand, P. Brands, and W. Steenbergen, "Handheld probe integrating laser diode and ultrasound transducer array for ultrasound/photoacoustic dual modality imaging," Optics express 22(21), 2636526374 (2014).

[12] A. Hariri, A. Fatima, N. Mohammadian, S. Mahmoodkalayeh, M. A. Ansari, N. Bely, and M. R. Avanaki, "Development of low-cost photoacoustic imaging systems using very low-energy pulsed laser diodes," Journal of biomedical optics 22(7), 075001 (2017).

[13] X. Dai, H. Yang, and H. Jiang, "In vivo photoacoustic imaging of vasculature with a low-cost miniature light emitting diode excitation," Optics letters 42(7), 1456-1459 (2017).

[14] P. K. Upputuri, and M. Pramanik, "Performance characterization of low-cost, highspeed, portable pulsed laser diode photoacoustic tomography (PLD-PAT) system," Biomedical optics express 6(10), 4118-4129 (2015).

[15] T. Petrosyan, M. Theodorou, J. Bamber, M. Frenz, and M. Jaeger, "Fast scanning widefield clutter elimination in epi-optoacoustic imaging using comb-LOVIT," in Ultrasonics Symposium (IUS), 2017 IEEE International (IEEE, 2017), pp. 1-1.

[16] M. Jaeger, L. Siegenthaler, M. Kitz, and M. Frenz, "Reduction of background in optoacoustic image sequences obtained under tissue deformation," Journal of biomedical optics 14(5), 054011 (2009).

[17] T. Petrosyan, M. Theodorou, J. Bamber, M. Frenz, and M. Jaeger, "Rapid scanning wide-field clutter elimination in epi-optoacoustic imaging using comb LOVIT," Photoacoustics 10, 20-30 (2018). 
[18] H. N. Y. Nguyen, and W. Steenbergen, "Reducing artifacts in photoacoustic imaging by using multi-wavelength excitation and transducer displacement," Biomedical Optics Express 10(7), 3124-3138 (2019).

[19] H. Y. Nguyen, and W. Steenbergen, "Out-of-plane artifact removal in photoacoustic imaging using transducer array displacement," in Opto-Acoustic Methods and Applications in Biophotonics IV (International Society for Optics and Photonics, 2019), p. $110770 \mathrm{P}$.

[20] S. Vaithilingam, T.-J. Ma, Y. Furukawa, I. O. Wygant, X. Zhuang, A. De La Zerda, O. Oralkan, A. Kamaya, R. B. Jeffrey, and B. T. Khuri-yakub, "Three-dimensional photoacoustic imaging using a two-dimensional CMUT array," IEEE transactions on ultrasonics, ferroelectrics, and frequency control 56(11), 2411-2419 (2009).

[21] Y. Wang, Z. Guo, L. V. Wang, T. N. Erpelding, L. Jankovic, J.-L. Robert, and G. David, "In vivo three-dimensional photoacoustic imaging based on a clinical matrix array ultrasound probe," Journal of Biomedical Optics 17(6), 061208 (2012).

[22] Y. Tan, K. Xia, Q. Ren, and C. Li, "Three-dimensional photoacoustic imaging via scanning a one dimensional linear unfocused ultrasound array," Optics express 25(7), 8022-8028 (2017).

[23] D. Wang, Y. Wang, Y. Zhou, J. F. Lovell, and J. Xia, "Coherent-weighted threedimensional image reconstruction in linear-array-based photoacoustic tomography," Biomedical optics express 7(5), 1957-1965 (2016).

[24] C. D. Herickhoff, M. R. Morgan, J. S. Broder, and J. J. Dahl, "Low-cost volumetric ultrasound by augmentation of 2D systems: Design and prototype," Ultrasonic imaging 40(1), 35-48 (2018).

[25] M. Jaeger, S. Schüpbach, A. Gertsch, M. Kitz, and M. Frenz, "Fourier reconstruction in optoacoustic imaging using truncated regularized inverse k-space interpolation," Inverse Problems 23(6), S51 (2007).

[26] G. Matrone, A. S. Savoia, G. Caliano, and G. Magenes, "The delay multiply and sum beamforming algorithm in ultrasound B-mode medical imaging," IEEE transactions on medical imaging 34(4), 940-949 (2014). 
[27] H. B. Lim, N. T. T. Nhung, E.-P. Li, and N. D. Thang, "Confocal microwave imaging for breast cancer detection: Delay-multiply-and-sum image reconstruction algorithm," IEEE Transactions on Biomedical Engineering 55(6), 1697-1704 (2008).

[28] R. Siphanto, K. Thumma, R. Kolkman, T. Van Leeuwen, F. De Mul, J. Van Neck, L. Van Adrichem, and W. Steenbergen, "Serial noninvasive photoacoustic imaging of neovascularization in tumor angiogenesis," Optics express 13(1), 89-95 (2005).

[29] A. Hauptmann, F. Lucka, M. Betcke, N. Huynh, J. Adler, B. Cox, P. Beard, S. Ourselin, and S. Arridge, "Model-based learning for accelerated, limited-view 3-d photoacoustic tomography," IEEE transactions on medical imaging 37(6), 1382-1393 (2018).

[30] C. Cai, X. Wang, K. Si, J. Qian, J. Luo, and C. Ma, "Streak artifact suppression in photoacoustic computed tomography using adaptive back projection," Biomed. Opt. Express 10(9), 4803-4814 (2019).

[31] J. I. Sperl, K. Zell, P. Menzenbach, C. Haisch, S. Ketzer, M. Marquart, H. Koenig, and M. W. Vogel, "Photoacoustic image reconstruction-A quantitative analysis," in European Conference on Biomedical Optics (Optical Society of America, 2007), p. $6631 \_6632$. 



\title{
6. Feasibility of Identifying Reflection Artifacts in Photoacoustic Imaging Using Two-Wavelength Excitation ${ }^{5}$
}

\begin{abstract}
One of the remaining challenges of bringing photoacoustic imaging to clinics is the occurrence of reflection artifacts. Previously, we proposed a method using multi-wavelength excitation to identify and remove the RAs. However, this method requires at least 3 wavelengths. Here we improve the method further by reducing the required number of wavelengths to 2 . We experimentally demonstrate this new method and compare it with the previous one. Results show that this new method holds great feasibility for identifying reflection artifacts in addition to preserving all advantages of the previous method.
\end{abstract}

\subsection{Introduction}

The identification of artifacts still remains as one of the main challenges of the reliability of photoacoustic imaging (PAI). Among of the artifacts, reflection artifacts (RAs), also called in-plane artifacts, and out-of-plane artifacts appear as real image features leading to image misinterpretation. While the occurrence of RAs is due to the acoustic inhomogeneity, out-of-plane artifacts are caused by strong absorbers located outside the imaging plane. Identification, and by preference correction these artifacts is therefore of importance, especially when this technique is to be widely used for clinical applications [1-3]. In this work, we aim to identify RAs (in-plane artifacts).

Recently, we proposed a method to remove RAs using multi-wavelength excitation [4, 5]. In the reported work, we used 4 [4] or 8 [5] wavelengths to image the sample and then

\footnotetext{
5 This chapter has been published as: H. N. Y. Nguyen, and W. Steenbergen, "Feasibility of identifying reflection artifacts in photoacoustic imaging using two-wavelength excitation," Biomedical Optics Express, 11(10), 5745-5759 (2020).
} 
compared the spectral response of the real image features and their RAs. The method provides considerable advantages over existing methods such as it does not require ultrasound (US) images as in the case of [6-8], sufficient training and experience of the user as in the case of [7,9], or a neural network trained with simulated data as in [10]. However, though the method was demonstrated with a compact and low cost PAI system, it still has specific requirements, such as at least 3 wavelengths are required, which are not widely available. In contrast, a 2-wavelength PAI system is a current PAI research focus [2, 11, 12]. In this paper, we aim to reduce the required number of wavelengths to 2 in addition to preserving all advantages of the previous method.

By imaging the sample with 2 wavelengths, we obtain the 2-wavelength response of the image features. We assume that RAs have a similar 2-wavelength response as their real image feature. Additionally, RAs appear at a larger depth with weaker signal than their original image feature. Combining these findings, we can thus identify the RAs.

In a simulation we will test the above assumption for robustness against acoustic attenuation, and we will demonstrate the method with phantom and in vivo experiments. Results show the feasibility of this method for identifying RAs in PAI. The results also show that this method outperforms the previous one [4] in terms of processing time. The size and cost of the PAI system can be further reduced thanks to a smaller number of wavelengths required.

\subsection{Method}

In our previous work [4], we imaged the sample with 4 different wavelengths. Extracting information from images corresponding to those wavelengths giving spectral responses of the image features. We assumed that RAs have a similar spectral response as their original real image feature. The similarity was quantified by using the Pearson correlation coefficient between the spectral responses. The Pearson correlation coefficient requires at least 3 data points ( 3 wavelengths) to give a meaningful coefficient. Therefore, 2 wavelengths are not sufficient with this approach.

In this work, the acquired PA images are first divided by the output energy of the corresponding wavelength. We then obtain the 2-wavelength responses of the image features in the same way as obtaining spectral responses presented in [4]. The 2-wavelength 108 
responses are normalized to their maximum. We define slope $s$ of a normalized 2wavelength response as: $s \equiv p\left(\lambda_{2}\right)-p\left(\lambda_{1}\right)$, where $p\left(\lambda_{1}\right)$ and $p\left(\lambda_{2}\right)$ are the normalized pixel values at 2 wavelengths $\lambda_{1}$ and $\lambda_{2}$ respectively. Since the values are normalized to the maximum, either $p\left(\lambda_{1}\right)$ or $p\left(\lambda_{2}\right)$ is 1 resulting in $s$ varying between -1 and 1 .

Fig. 6.1 shows a normalized 2-wavelength response example of an image feature, the red line. As RAs have a similar spectral response as their original real image feature [4], we assume that the slope of RAs is close to the slope of their real image feature. A threshold, $\Delta s_{t h}$, is used to determine the closeness. If an image feature has a slope of $s$, the slope of its RAs must be in between $s-\Delta s_{t h}$ and $s+\Delta s_{t h}$. The light blue area in Fig. 6.1 depicts the possible slope of RAs of that image feature. In this work, we use the term "slope difference", $\Delta s_{i, j}=\left|s_{i}-s_{j}\right|$, to refer to the difference of the slope of image features $i$ and $j$. If feature $i$ or feature $j$ is an RA of the other, $\Delta s_{i, j}<\Delta s_{t h}$. On the other hand, since $s$ varies between -1 and $1, \Delta s$ is then between 0 and 2 .

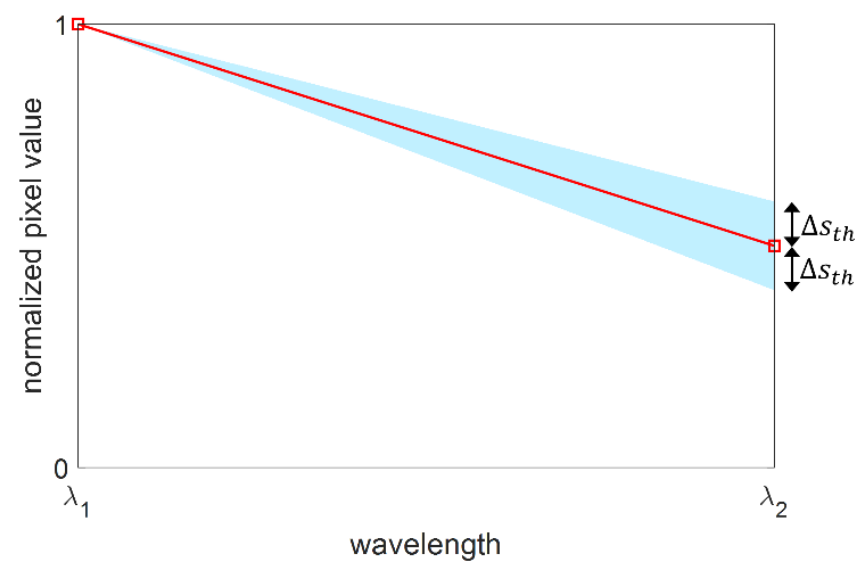

Fig. 6.1. 2-wavelength response and its margins for identifying RAs.

It is worth reminding that RAs appear at a larger depth and have weaker signal than the original image feature [4]. Combining this with the slope difference, RAs can therefore be identified.

Fig. 6.2 shows the flowchart of the method. It is similar to the one presented in [4] except the "Calculate correlation coefficients" step replaced by "Calculate $\Delta s$ ". Of the 2 acquired 
images corresponding to the 2 wavelengths, the image having higher signal is selected for segmentation. The segmentation algorithm, based on the Sobel edge detection algorithm, is to detect image features and thus obtain their 2-wavelength responses. These 2 -wavelength responses are processed in order to identify RAs.

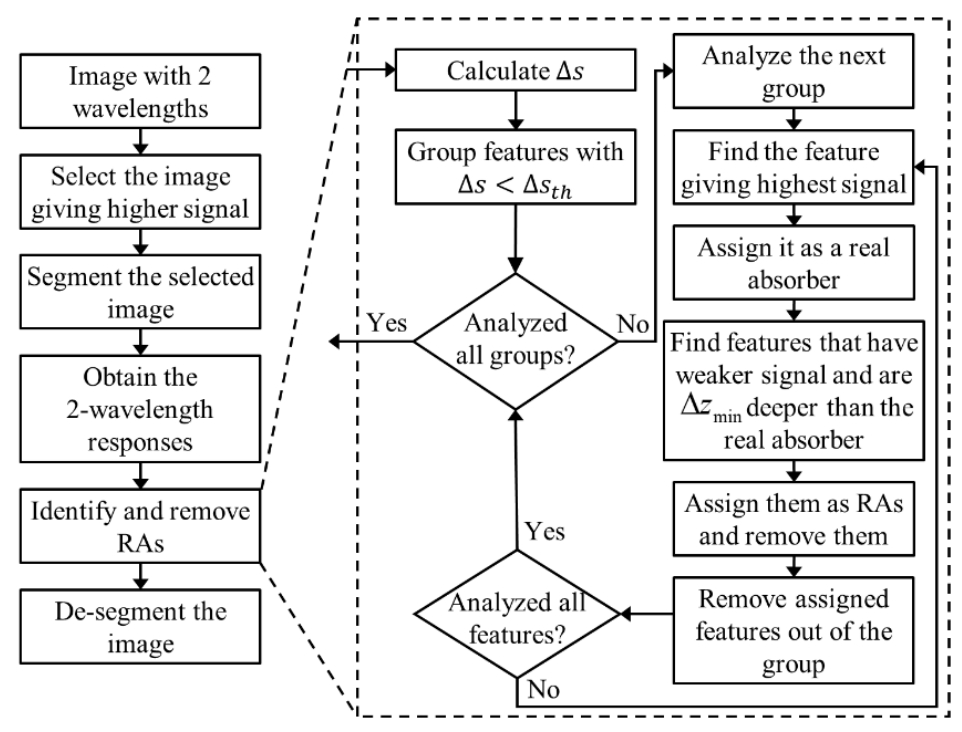

Fig. 6.2. Flowchart of the method.

$\Delta z_{\text {min }}$ in the flowchart is a minimum distance between an RA and its real image feature. This is to avoid the situation when 2 identical absorbers are close to each other, they might have similar 2-wavelength responses, consequently, one might be identified as an RA of the other. In other words, the method only looks for RAs of an image feature in the region below it at least $\Delta z_{\min }$. We performed a $\Delta z_{\text {min }}$ study in our previous work [4], we will apply the same value $\Delta z_{\min }=1.7 \mathrm{~mm}$ in this work. $\Delta s_{t h}$ is empirically set 0.05 and will be discussed further in the Discussion section.

As reported in [4], the RA identification can also be achieved without using segmentation. In this approach, the slope of each image pixel is compared to all other pixels. Fig. 6.3 shows an example of this approach. Fig. 6.3(a) and (b) show 2 PA images of a finger at 808 and $940 \mathrm{~nm}$ respectively. A slope map of all pixels is calculated based on these 2 images, seen in Fig. 6.3(c). All slopes are then compared to each other pixel by pixel. Further detail of this approach will be described in section 6.4.4. 


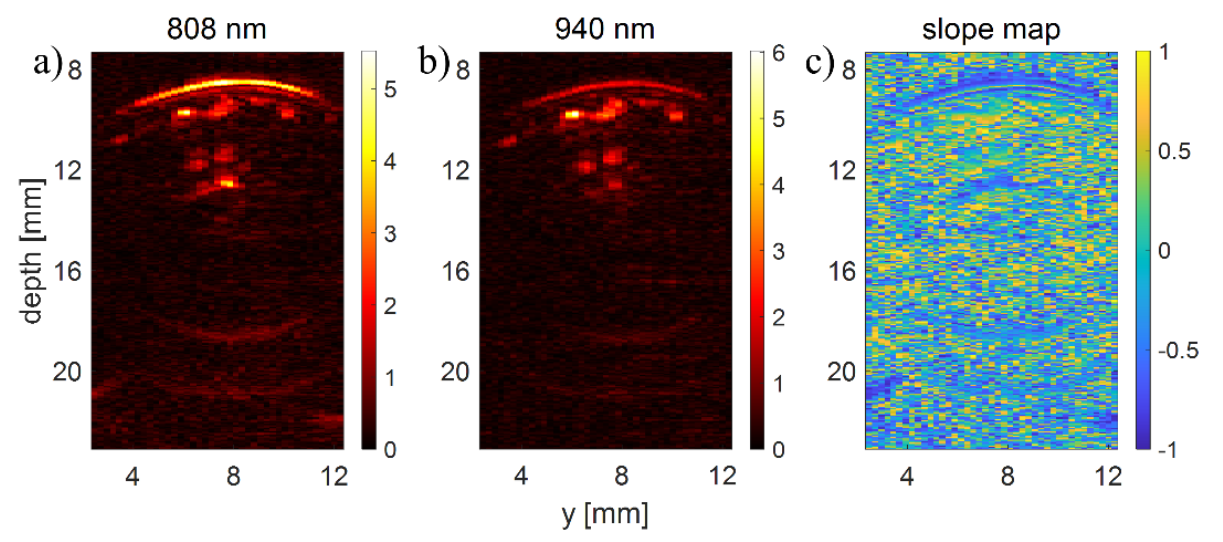

Fig. 6.3. Slope map of an in vivo experiment. PA image of a finger at $808 \mathrm{~nm}$ (a) and $940 \mathrm{~nm}$ (b). Slope map of all pixels calculated from the 2 images in (a) and (b).

\subsection{Simulation}

In principle, the method is based on the assumption that RAs have a similar 2wavelength response as their real image feature. In other words, the direct and reflected US waves still carry the optical properties of the absorber along the propagation despite of acoustic attenuation. This assumption may not be valid in cases where the two optical wavelengths generate PA waves in completely different acoustic spectral ranges, and where the broader bandwidth signal suffers significantly more from acoustic attenuation than the narrower bandwidth. To verify this assumption, we performed a simulation using ValoMC [13] which is based on the Monte Carlo and k-Wave toolbox.

We simulated photoacoustic imaging at 2 different wavelengths of a blood vessel above a piece of Perspex in a soft tissue medium. The yellow round and yellow rectangle objects in Fig. 6.4(a) are the blood vessel with a diameter of $1 \mathrm{~mm}$ and the Perspex piece with a thickness of $10 \mathrm{~mm}$. The illumination is vertically from the top center with a beam width of $2 \mathrm{~mm}$ and the detection is a line of 64 point detectors at $\mathrm{z}=0 \mathrm{~mm}$ with a total length of 7.4 $\mathrm{mm}$. As the acoustic attenuation might affect the US waves carrying the optical information of the absorber, especially at high acoustic frequencies, we chose 2 excitation wavelengths to generate signals with different acoustic spectra. The first wavelength is $532 \mathrm{~nm}$. As hemoglobin is strongly absorbing at this wavelength, light can only penetrate into small part of the lumen generating high frequency US waves, see in Fig. 6.4(b) the initial pressure at 
$532 \mathrm{~nm}$. The second wavelength is $800 \mathrm{~nm}$ to generate lower frequency US waves, see in Fig. 6.4(c) the initial pressure at $800 \mathrm{~nm}$.
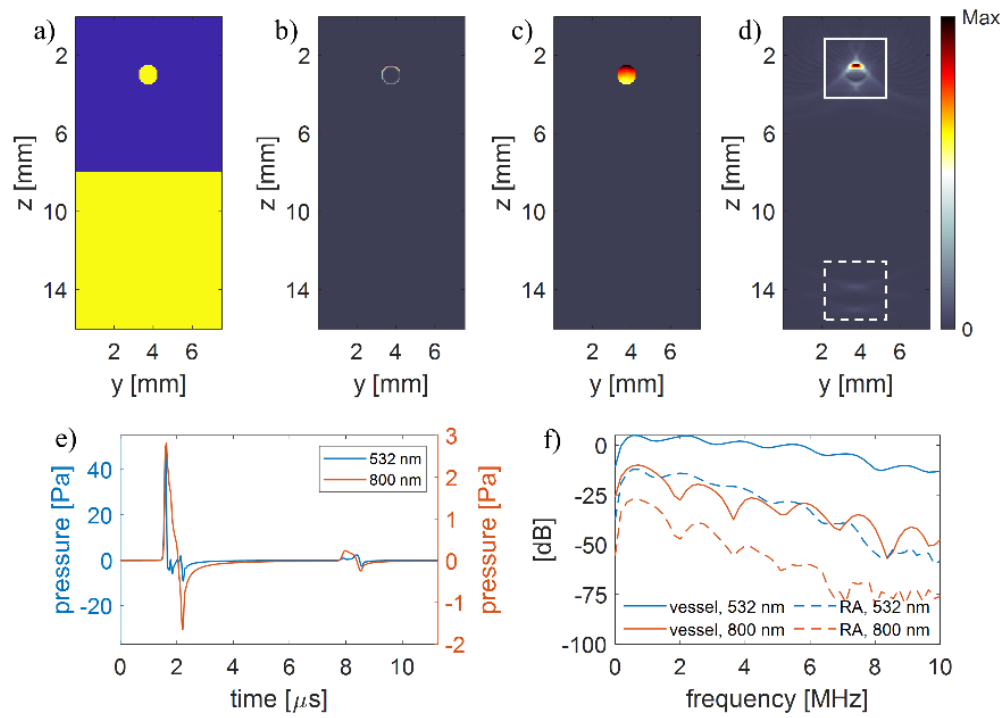

Fig. 6.4. Photoacoustic imaging simulation. (a) Simulation objects. Yellow round and yellow rectangle objects are a blood vessel and a piece of Perspex, respectively. (b) and (c): Initial pressure at 532 and $800 \mathrm{~nm}$ respectively. (d) Reconstructed image at $532 \mathrm{~nm}$. (e) Recorded signal at the center detector at 532 and $800 \mathrm{~nm}$. (f) Spectrum of the US signal of the blood vessel and its RA at 532 and $800 \mathrm{~nm}$. Solid and dashed rectangles in (d) are an image of the blood vessel and its RA.

We simulated the blood vessel with an oxygen saturation level of $90 \%$ which has an absorption coefficient of 233 and $4.3 \mathrm{~cm}^{-1}$ at 532 and $800 \mathrm{~nm}$ respectively [14]. The absorption coefficient elsewhere is $0.01 \mathrm{~cm}^{-1}$. The reduced scattering coefficient of the medium is 12.3 and $8.4 \mathrm{~cm}^{-1}$ at 532 and $800 \mathrm{~nm}$ respectively [14] mimicking soft tissue. The blood vessel and the medium have the same speed of sound of $1575 \mathrm{~m} / \mathrm{s}$ and density of 1065 $\mathrm{kg} / \mathrm{m}^{3}$ [15] while these values in the Perspex piece are $2730 \mathrm{~m} / \mathrm{s}$ and $1180 \mathrm{~kg} / \mathrm{m}^{3}$. The acoustic attenuation coefficient in the medium is frequency dependent and modelled as $\alpha=a f^{b}$, where $a=0.56 \mathrm{~dB} / \mathrm{cm} / \mathrm{MHz}$ and $b=1[16]$.

Fig. 6.4(d) is a reconstructed image at $532 \mathrm{~nm}$. The solid and dashed rectangles in Fig. 6.4(d) are the image of the blood vessel and its RA. Fig. 6.4(b)-(d) share the same color bar. Fig. 6.4(e) shows the recorded signal at the center detector at the wavelengths of 532 and 112 
$800 \mathrm{~nm}$ and Fig. 6.4(f) shows the spectrum of the US waves of the blood vessel and its RA at 532 and $800 \mathrm{~nm}$. The oscillation in the spectrum is similar to the observation in [17]. The spectrum of the signal at the wavelength of $532 \mathrm{~nm}$ is broader and thus experiences attenuation differently compared to the signal at the wavelength of $800 \mathrm{~nm}$.

The slopes of the blood vessel's and RA's response are -0.937 and -0.941 respectively. It can be seen that though the reflected US waves experience stronger attenuation due to a longer propagation path (10 $\mathrm{mm}$ in this simulation) affecting their acoustic spectrum, they still carry the optical information as in the direct US waves. We repeated the simulation with the Perspex piece repositioned $5 \mathrm{~mm}$ deeper resulting in a propagation path of the reflected US waves $20 \mathrm{~mm}$ longer than the direct US waves. The slope of the RA's response in this case is -0.934 which is still highly close to the slope of the blood vessel's response. These simulation results strongly enhance our assumption.

We also performed another simulation similar to the one above but without the Perspex layer. A new blood vessel identical to the one above was placed at the position of the RA in the previous simulation. The slopes of the response of the top and the bottom blood vessels are -0.932 and -0.879 respectively. The slope difference is 0.053 and with $\Delta s_{t h}=0.05$, the proposed method still can distinguish these 2 identical absorbers. The slope difference would be even larger for optically different absorbers showing that the method will not mis-identify a real absorber as an RA of another one.

It is worth noting that this simulation did not take into account noise and the spectral response of the detectors and the medium was optically and acoustically homogeneous. In practice, these aspects might affect the 2 -wavelength response of the image features.

\subsection{Experimental results}

To experimentally validate the method, we use the same datasets presented in [4]. The data was acquired using a handheld PAI probe [4]. It consists of laser diodes emitting laser light at 4 different wavelengths $808,915,940$ and $980 \mathrm{~nm}$ with output pulse energy of 0.96 , $0.98,0.89$ and $0.82 \mathrm{~mJ}$ respectively. In the experiments. the laser diodes were triggered at a repetition rate of $1 \mathrm{kHz}$. The transducer array has a center frequency of $7.5 \mathrm{MHz}$ with a bandwidth of $100 \%$. The array contains 128 elements with a pitch of $0.245 \mathrm{~mm}$. The center 64 elements were used in the experiments. 
Each dataset contains 4 PA images at the 4 wavelengths and 1 US image averaged over 100 pulses. Of these 4 wavelengths, we select two. In our previous work [4], of all images, the image having the strongest signal was used for segmentation. In this work, the image for segmentation is selected from the images of the 2 wavelengths.

\subsubsection{Phantom}

Fig. 6.5 shows a phantom experiment. A schematic cross-section and a photo of the phantom are given in Fig. 6.5(a) and Fig. 6.5(b) respectively. Four black suture wires (USP 4/0, diameter of $0.2 \mathrm{~mm}$, Vetsuture Nylon, The Netherlands) are to mimic four absorbers. We used the same material for the absorbers in order to verify if the method would misidentify a real absorber as an RA of another absorber. Underneath absorbers 3 and 4 is a Perspex plate with a thickness of $1 \mathrm{~mm}$ mimicking an acoustic reflector. The coupling medium was a solution of $3.5 \%$ Intralipid $20 \%$ in demi-water with an estimated $\mu_{s}^{\prime}=6 \mathrm{~cm}^{-}$ ${ }^{1}$ at the wavelength of $900 \mathrm{~nm}$ [18]. Fig. 6.5(c) shows a PA and US combined image. The gray color part is the US image showing two surfaces of the plate. The hot color part is the PA image at $808 \mathrm{~nm}$. In the PA image, underneath the plate are RAs of the absorbers above the plate.

Fig. 6.5(d) presents the spectral response of several image features depicted in Fig. 6.5(c). The spectral responses are normalized to their maximum. Fig. 6.5(e) shows the RA corrected image using all 4 wavelengths [4]. The results again prove the capability of reducing RAs of the method in [4]. 


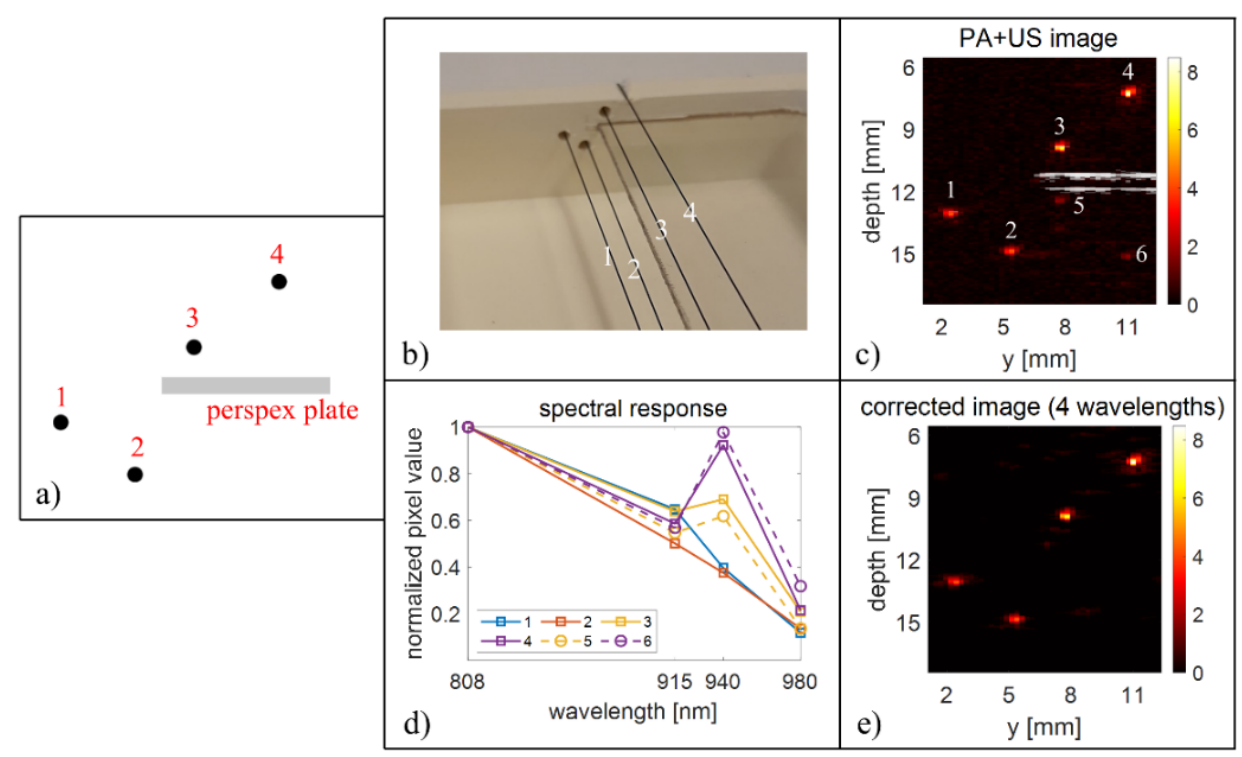

Fig. 6.5. Phantom analysis. (a) schematic cross-section of the phantom. (b) Combined PA and US image of the phantom. (c) Segmented image with features numbered. (d) Spectral response of image features depicted in (c). (e) RA corrected image using 4 wavelengths.

Fig. 6.6 shows results of identifying RAs using 2 wavelengths: 915 and $940 \mathrm{~nm}$ (Fig. 6.6(a-b)) and 940 and $980 \mathrm{~nm}$ (Fig. 6.6(c-d)). Figures in the left column (Fig. 6.6(a) and (c)) are 2-wavelength responses of the image features under consideration. The light orange and violet areas present the possible slope of features 3 and 4's RAs respectively. Figures in the right column (Fig. 6.6(b) and (d)) are RA corrected images.

In the approach using 915 and $940 \mathrm{~nm}, \Delta s_{3,5}=0.0365$ and $\Delta s_{4,6}=0.0492$, features 5 and 6 are then identified as an RA of features 3 and 4 respectively. This is in great agreement with the result using 4 wavelengths showing the feasibility of identifying RAs with these two wavelengths. In contrast, in the approach using 940 and $980 \mathrm{~nm}$, most of the slopes are in the light orange area, seen in Fig. 6.6(c). As a consequence, features 1, 2, 5, 6 are also identified as RAs of feature 3, seen in Fig. 6.6(d). This result indicates that not all combinations of two wavelengths are appropriate for RA identification. 


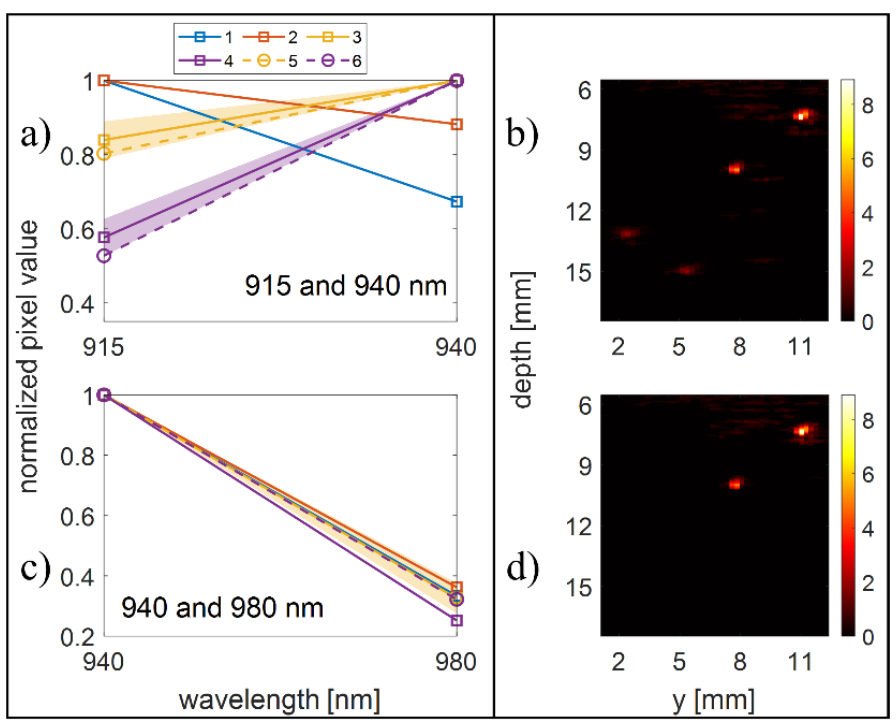

Fig. 6.6. Identifying RAs using 2 wavelengths: 915 and $940 \mathrm{~nm}(\mathrm{a}-\mathrm{b}) ; 940$ and $980 \mathrm{~nm}$ (c-d). (a)(c) 2-wavelength responses of image features. (b)(d) RA corrected images using 2 wavelengths.

In this phantom study, the image at $940 \mathrm{~nm}$ has the highest signal among the selected wavelengths and is used for segmentation in the above approach using 2 wavelengths.

\subsubsection{In vivo 1}

Fig. 6.7 shows the analysis presented in [4]. Fig. 6.7(a) is an acquired PA image showing a cross-sectional view of a finger. The image is then processed for RA removal using 4 wavelengths, seen in Fig. 6.7(b). Fig. 6.7(c) shows the segmented image with a few features numbered. Features 4, 5 and 6 are RAs of features 2, 3, and 1 respectively [4]. The spectral responses of these features are depicted in Fig. 6.7(d). 


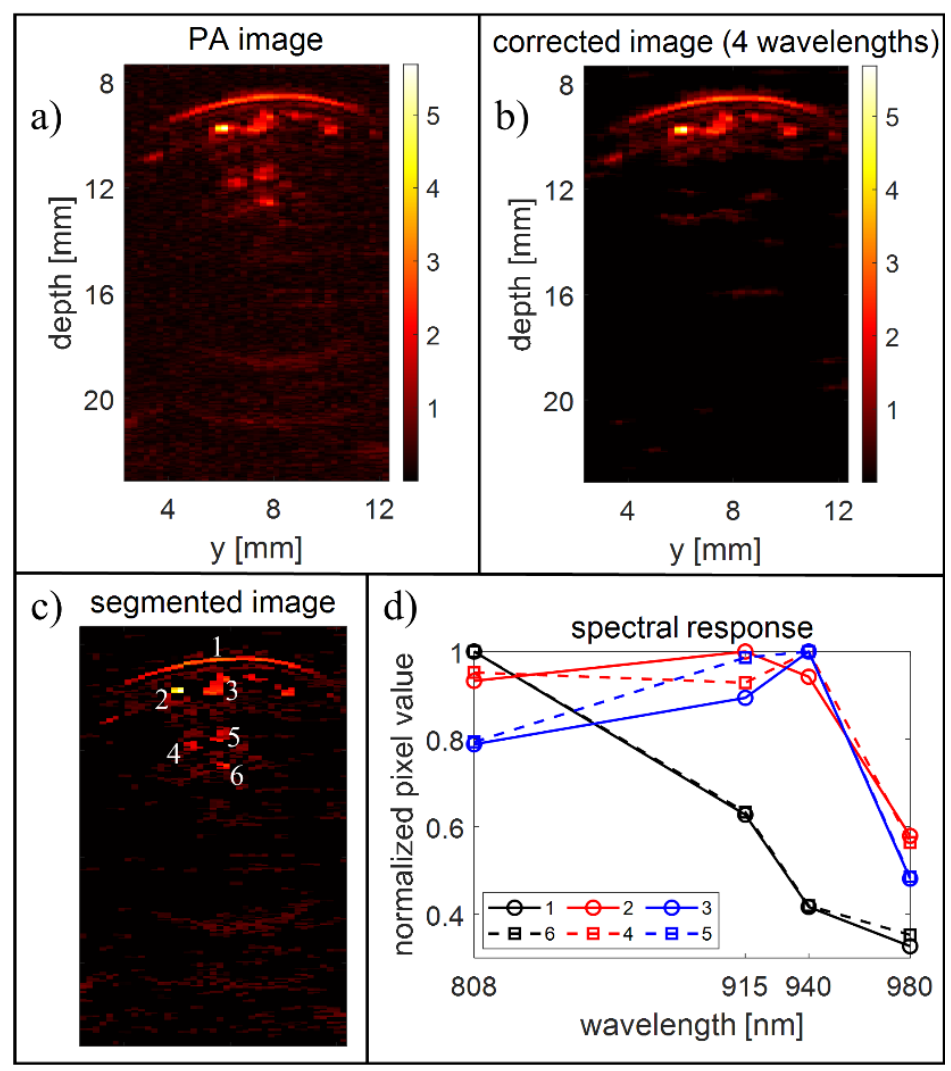

Fig. 6.7. In vivo image analysis. (a) Acquired PA image. (b) RA corrected image. (c)

Segmented image with features numbered. (d) Spectral response of image features depicted in (c).

Fig. 6.8 presents results of RA identification using 2 wavelengths: 808 and $940 \mathrm{~nm}$ (Fig. 6.8(a-b)) and 808 and $915 \mathrm{~nm}$ (Fig. 6.8(c-d)). Figures in the left column show the 2wavelength response of the image features under consideration while figures in the right column show RA corrected images.

The result of using 2 wavelengths (808 and $940 \mathrm{~nm}$ ) is similar to the one using 4 wavelengths (Fig. 6.7(b)). This enhances the feasibility of identifying RAs using 2 wavelengths shown in the phantom study. However, as mentioned in the phantom study, not all 2-wavelength combinations could work properly. Fig. 6.8(d) shows an example that using 2 wavelengths might miss some RAs. 
In this in vivo study, the image at $915 \mathrm{~nm}$ is selected for segmentation. However, this image is not included in the approach using 808 and $940 \mathrm{~nm}$. The image at 940 is then used for segmentation in this approach resulting in a slightly different segmented image and feature numbering. This difference does not affect the method. However, for convenience and simplicity of presenting the spectral response, we refer to the same image features, shown in Fig. 6.7(c), in all approaches.

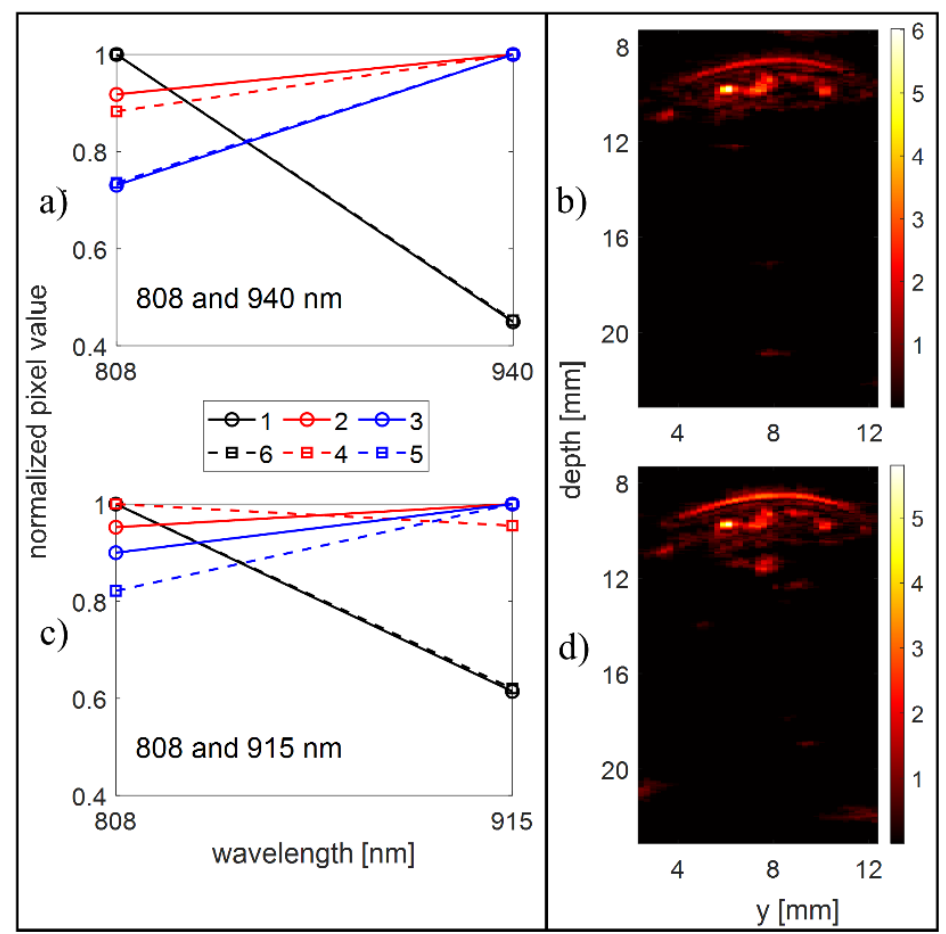

Fig. 6.8. Identifying RAs using 2 wavelengths: 808 and $940 \mathrm{~nm}(\mathrm{a}-\mathrm{b}) ; 808$ and $915 \mathrm{~nm}$ (c-d). (a)(c) 2-wavelength responses of image features. (b)(d) RA corrected images using 2 wavelengths.

\subsubsection{In vivo 2}

In both phantom and in vivo studies presented above, the approach using 808 and 940 $\mathrm{nm}$ provides the most reasonable RA identification while any other 2-wavelength combinations either mis- or over-identify RAs. To verify the performance of this combination, we carried out another in vivo experiment with the same protocol as in vivo 1 described in [4]. 
Fig. 6.9 presents the result of this experiment. Similar to the in vivo 1 study, Fig. 6.9(a) shows a PA image of a finger. This image is then RA processed using 4 wavelengths (Fig. 6.9(b)) or 2 wavelengths (Fig. 6.9(c-d)). Fig. 6.9(c) and (d) are obtained by using 808 and $940 \mathrm{~nm}$ and 808 and $980 \mathrm{~nm}$ respectively. In this experiment, the image acquired at $808 \mathrm{~nm}$ is used for segmentation, therefore, the result of all approach are presented based on the same image.

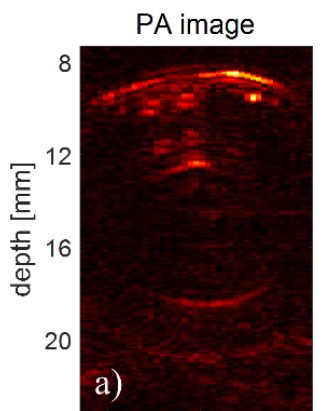

$4 \quad 8 \quad 12$

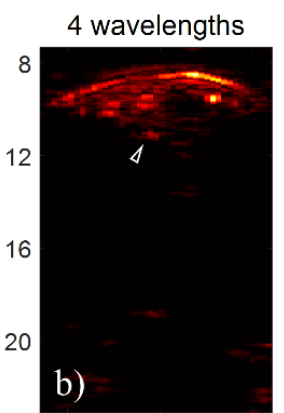

4

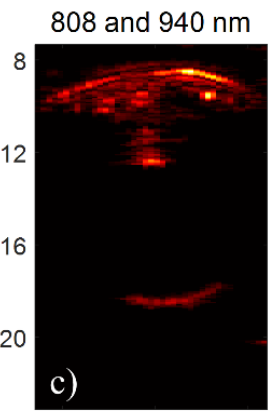

8
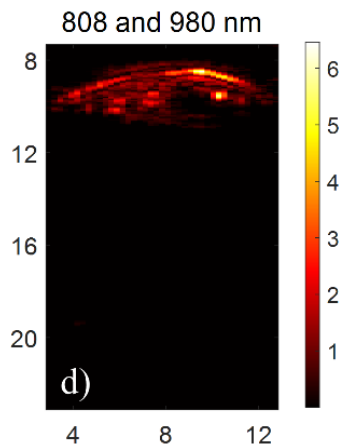

$\mathrm{y}[\mathrm{mm}]$

Fig. 6.9. In vivo image (a) with RAs corrected using 4 wavelengths (b), and 2 wavelengths (c-d). White arrow indicates an unremoved RA.

In the approach using 4 wavelengths, while most of the RAs are removed, one RA, marked with a white arrow, is not. This is probably due to the noise as discussed in [4]. While using 808 and $940 \mathrm{~nm}$ works reasonably well in the studies in sections 6.4.1 and 6.4.2, this approach is underperforming in this case leaving a few RAs unremoved, seen in Fig. 6.9(c). However, the approach using 808 and $980 \mathrm{~nm}$ provides a corrected image with all RAs identified. It even slightly outperforms the approach using 4 wavelength as the marked RA is also identified. These observations indicate that the approach using 2 wavelengths is strongly affected by the noise and the choice of 2 particular wavelengths is of importance to achieve a reasonable RA identification.

\subsubsection{RA identification without segmentation}

As described in section 6.2, the RA identification can also be achieved without using segmentation. The image processing is the same as presented in [4]. 
Fig. 6.10(a) shows again the PA image of the in vivo 1 experiment (section 6.4.2). Fig. 6.10(b) shows the spectral slope value of all pixels (see also Appendix 1.3). From the similarity in spectral slope of the skin and of deeper structures, we can already identify RAs belonging to the skin signal. Fig. 6.10(c) illustrates a pixel (top yellow pixel) in the skin. The slope of the two wavelength spectral response ( 808 and $940 \mathrm{~nm}$ ) of this pixel is indicated by the blue line in the white dashed window in Fig. 6.10(a).
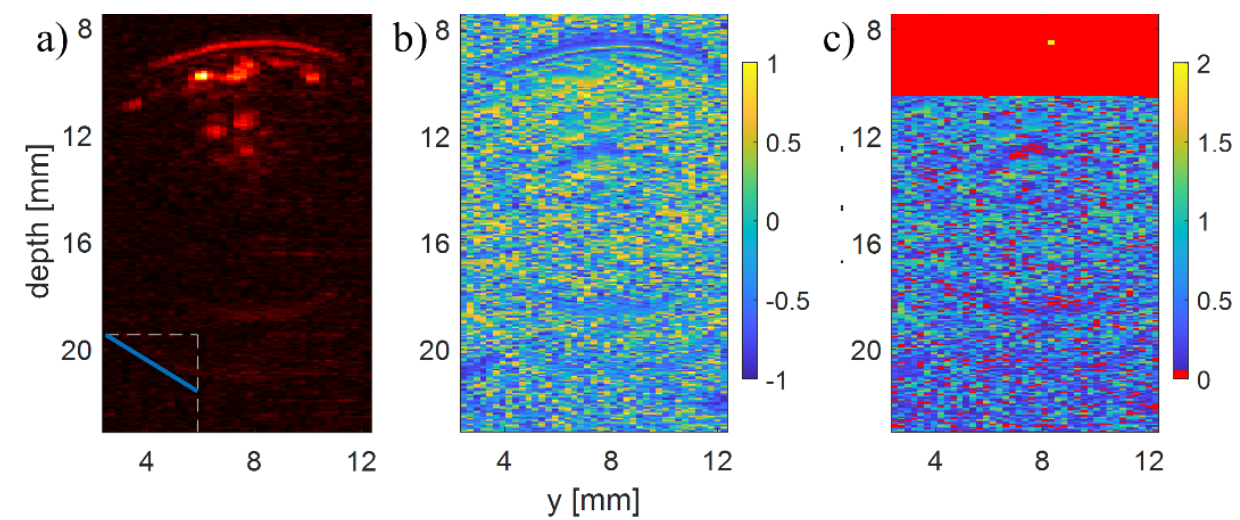

Fig. 6.10. RA identification with the non-segmentation method in the in vivo 1 image. (a) in vivo $1 \mathrm{PA}$ image. Blue line in the white dashed window is the slope of the reference pixel (top yellow pixel in (c)). (b) slope value map of all pixels. (c) $\Delta s$ map of a pixel in the skin with all other pixels at least $2 \mathrm{~mm}$ below the considered pixel (values below 0.05 are colored red).

The slope of the reference pixel is compared to other pixels at least $2 \mathrm{~mm}$ below it giving a $\Delta s$ map, seen in Fig. 6.10(c) and also in Appendix 2.20. The red color in Fig. 6.10(c) indicates $\Delta s$ below 0.05 revealing RAs (red pixels) of the considered pixel.

Fig. 6.11 shows the results of identifying RAs using 4 (Fig. 6.11(b) and (e)) or 2 (Fig. 6.11(c) and (f)) wavelengths without segmentation. Fig. 6.11(a) and (d) are acquired images of the phantom and in vivo 1 experiments respectively. The top and bottom images are results of the phantom and in vivo 1 experiments presented in section 6.4.1 and section 6.4.2. The 2 wavelengths used in the phantom experiment are 915 and $940 \mathrm{~nm}$, and in the in vivo 1 experiment are 808 and $940 \mathrm{~nm}$. All the images share the same color scale on the right. 

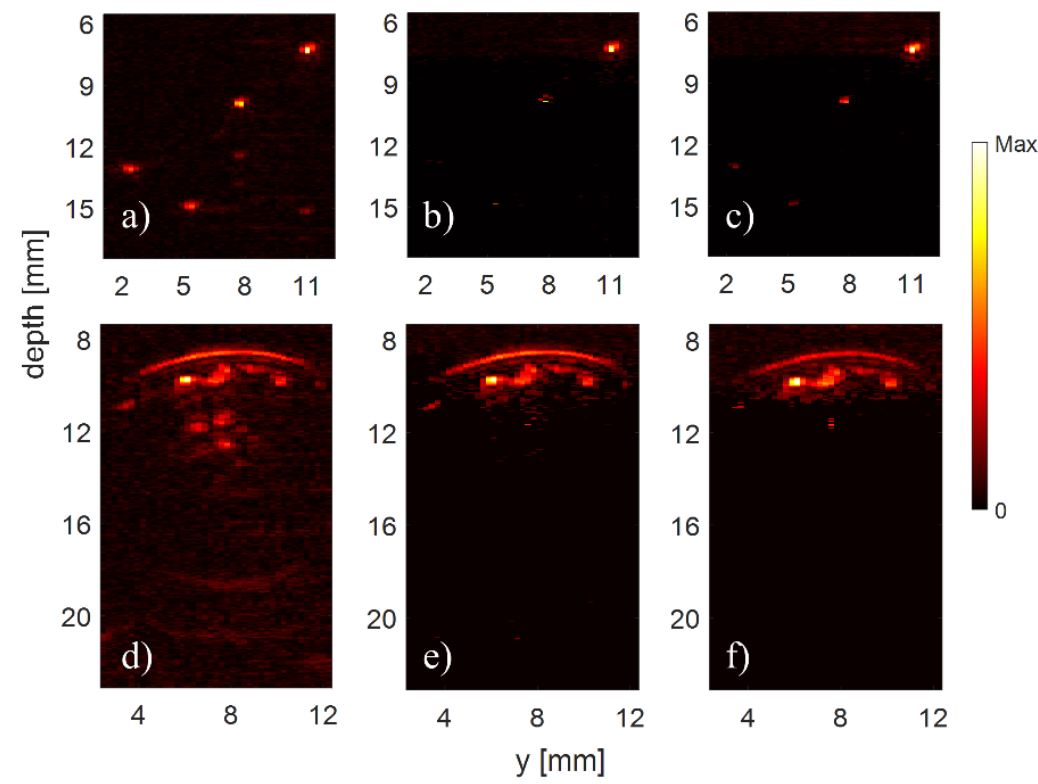

Fig. 6.11. Results of identifying RAs using 2 or 4 wavelengths without segmentation. Rows (top to bottom) images of the phantom and in vivo experiment presented in section 6.4.1 and section 6.4.2 respectively. Columns (left to right): acquired PA images, results of using 4 wavelengths [4], and results of using 2 wavelengths respectively.

In the phantom experiment, absorbers on the left are partly removed in the method using 4 wavelengths. This is similar to the observation in [4]. The result of 2 wavelengths is slightly better in this aspect. In the in vivo 1 experiment, the results of these 2 methods are in good agreement.

In the approach without using segmentation, most of the noisy pixels at large depths are identified as RAs. This can be also seen in Appendix 2.20 that while scanning through noisy pixels at the top of the image, noisy pixels at larger depths are identified as RAs of those pixels. At the end of the movie, most of noise pixels are removed. Our hypothesis is that for random noise, there would be always some other noisy pixels having a similar slope. To verify this, we create 2 images (same size as in vivo 1 images) with only random noise (command 'rand" in Matlab which generates uniformly distributed random numbers), Fig. 6.12(a) and (b), and treat them as 2 PA images. We then calculate a slope map based on these 2 images, seen in Fig. 6.12(c). Fig. 6.12(d) shows the result with the method without using 
segmentation. It can be seen that though the 2 initial images contain only noise, most of noisy pixels at large depths are still identified as RAs of their above noise. This result strengthens our hypothesis.
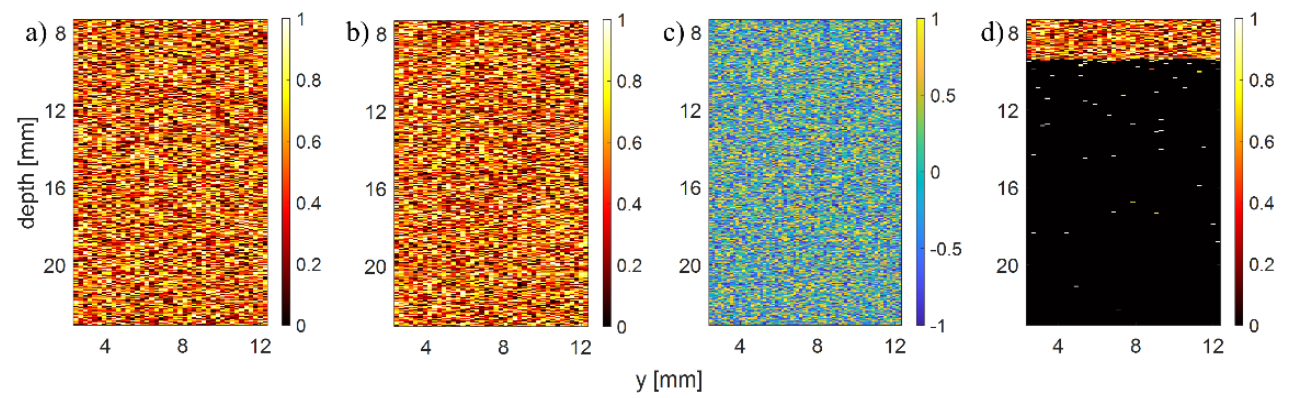

Fig. 6.12. RA identification for random noise. Random noise images (a) and (b). (c) Slope map formed based on imagesc (a) and (b). Corrected images without using segmentation.

It is worth noting that, RAs must have a pixel value smaller than their real absorber. Therefore, real images features at large depths having pixel value higher than the noise level will not be affected by the noisy pixels above them.

We also assess the performance of this method in low signal-to-noise ratio (SNR) images. Fig. 6.13(a) shows again the phantom image with a green and a dashed white rectangle depicting signal and noise windows respectively. The SNR is defined as:

$$
S N R=10 \log _{10}\left(\frac{E(S)}{E(N)}\right),
$$

where $E(S)$ and $E(N)$ are the mean square of the pixel values in signal and noise windows respectively. We manually add random noise to the phantom image using 'rand' in Matlab, seen in the upper row in Fig. 6.13. The lower row are RA corrected images. All the images have the same color scale shown at the bottom. We analyze the absorber with the second strongest signal rather than other absorbers with low signals so that we could have a large range of the SNR. 


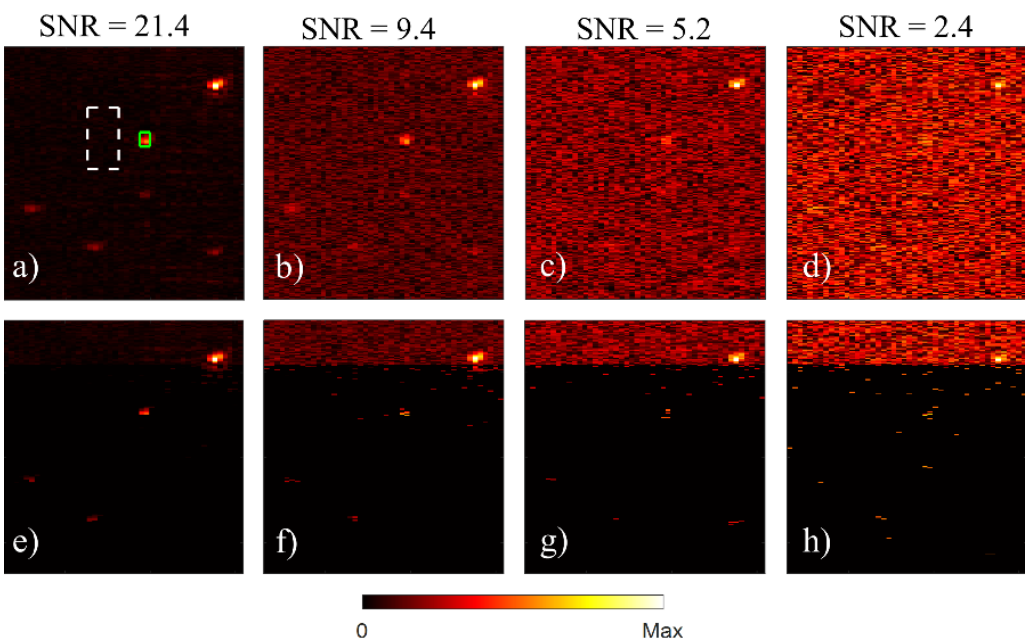

Fig. 6.13. Phantom images with different SNRs in $\mathrm{dB}$ (left to right). Upper row: PA images with random noise manually added. Lower row: RA corrected images corresponding the upper images. Green rectangle: signal window. Dashed white rectangle: noise window.

At the SNR is 9.4 and lower, RAs of the top right absorber have pixel values close to the noise level. The method can still remove them as RAs of either the top right absorber or above noisy pixels (as discussed above). When reducing the SNR to $5.2 \mathrm{~dB}$ and lower, the considered absorber is barely distinguishable from noise, it is then significantly removed. These results show that the method might not work properly at a certain SNR and lower.

To analyze the effect of the SNR on real image features at large depth, we count the number of remaining pixels in the signal window that have a greater pixel value than the noise level determined in the noise window. We repeat this analysis 3 times at each SNR. Fig. 6.14 shows the number of remaining pixels versus the SNR in an error bar plot presenting the average, minimum and maximum values. It can be seen that from the SNR equal and lower than around $6 \mathrm{~dB}$, the real image feature is significantly removed. 


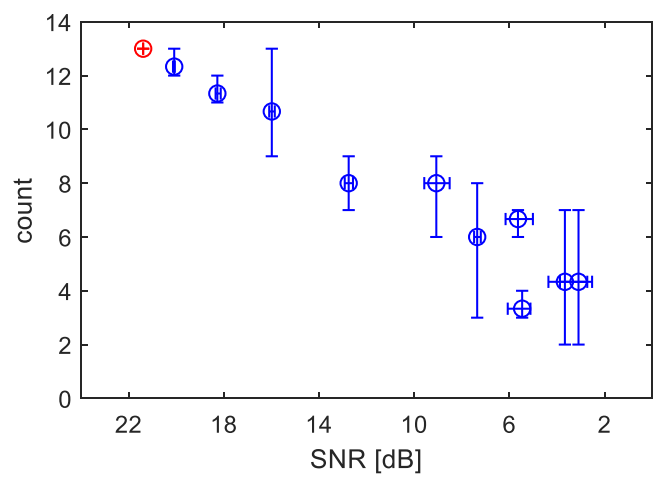

Fig. 6.14. Number of remaining pixels of a real image feature vs SNR. Red data point is when no noise added.

\subsection{Discussion}

PAI has great potential in clinical applications. Tackling RAs in PAI is therefore an important step to bring this technique further forwards. The proposed method offers significant advantages over existing methods for identifying RAs. First, the proposed method does not require any training or experience of the user as in [7, 9]. Second, it takes advantage of the use of 2-wavelength PAI which is a current focus of PAI [2, 11, 12]. Third, this method does not need US images to work as in [6-8]. Multi-plane wave US imaging comes at a greater time consuming and higher processing expense. Finally, compared to the method using 4 wavelengths [4], the 2-wavelength method is feasible with an even more compact and cost-efficient system.

In this work, we use the $\Delta z_{\text {min }}$ determined in the previous report [4]. $\Delta z_{\text {min }}$ defines a region below an original image feature in which the method will not identify any RAs of that feature. This still remains as one of the main limitations of the proposed method.

In the proposed method, $\Delta s_{t h}$ used to quantify the closeness between the 2-wavelength slopes is a crucial threshold. The larger $\Delta s_{t h}$ is, the higher the chance to identify RAs the method has. However, it is also a higher chance RA over-misidentification is such as real image features identified as RAs. In this work, $\Delta s_{t h}$ is set based on our experience to get reasonable results. 
Choosing the 2 wavelengths for a reasonable result is of importance as the method must distinguish: (1) different absorbers with the same optical absorption properties located at different locations and (2) different chromophores. For the former, the variance of the local light fluence along the depth (rather than other directions as we use $\Delta z_{\text {min }}$ ) must be significant enough so that the method will not mis-identify one absorber as an RA of another identical absorber. That means the optical efficient attenuation coefficient, $\mu_{e f f}=\sqrt{3 \mu_{a}\left(\mu_{a}+\mu_{s}^{\prime}\right)}$, of the bulk medium must not be flat at the chosen wavelengths. For the latter, the optical absorption of different chromophores must have different slopes at the chosen wavelengths. These together then require prior knowledge of the imaging sample. However, since the main chromophores in in vivo PAI are melanin and hemoglobin and based on their well-known optical absorption spectra [19], various wavelength combinations can be used. For example, the 2 wavelengths around 800 and $900 \mathrm{~nm}$ would be an option. The absorption of melanin in this range has a negative slope while oxygenated hemoglobin and deoxygenated hemoglobin have a positive slope and a relatively flat slope respectively. Therefore the method could differentiate these chromophores in this range. The $\mu_{\text {eff }}$ of the bulk tissue mainly depend on the absorption of the blood and scattering the soft tissue. The blood volume fraction in soft tissue is $1-8 \%$ [20]. The optical scattering of the soft tissue has a slightly negative slope [14]. If the oxygen saturation level of the blood is $60 \%$ or higher, the slope of the $\mu_{e f f}$ of the bulk medium is always positive with the 2 wavelengths of 800 $\mathrm{nm}$ and any wavelength in between 810-900 $\mathrm{nm}$. Therefore the method could distinguish identical absorbers.

It is shown in the experimental result section that in one case, a 2-wavelength combination works properly but another combination does not, and it is opposite in another case. This negatively affects the reliability of the proposed method though it still shows the feasibility of the method for identifying RAs. There would be 2 reasons for this. First, as presented in the experimental results, the slope formed by using 2 wavelengths is highly sensitive to noise. RA mis- or over-identification might happen with the proposed method. This can be seen in section 6.4.4 when the SNR of the image is too low. Second, the chosen 2 wavelengths might not differentiate well the optical absorption properties of the different 
absorbers as discussed in the strategy above. Further improvements in these aspects will be investigated in our future work.

As discussed above, choosing the 2 wavelengths might require prior knowledge about the imaging sample. If the optical properties of the sample change, choosing another 2wavelength combination might be needed for a reliable RA identification. However, in the wavelength range of $800-900 \mathrm{~nm}$, the change of the absorption of blood at different oxygen saturation levels is little. Using 2 wavelengths in this range would work well for RA identification in in vivo imaging.

As shown in [5], out-of-plane artifacts (with the acoustic source located outside the imaging plane) can also appear in the acquired PA images, these artifacts cannot be tackled using 4 wavelengths. This still holds for the proposed method. An additional method, such as transducer displacement $[5,9]$, is needed to identify these artifacts.

In the approach using segmentation, both methods using 4 or 2 wavelengths take $\sim 0.2$ $0.3 \mathrm{~s}$ time for correcting a PA image with a dimension of $512 \times 64$ pixels. The processing time in this case is approximately the same since most of the processing time is on the segmentation. However, in the approach without using segmentation, while the method using 4 wavelengths takes $\sim 2 \mathrm{~s}$, the method using 2 wavelengths only takes $0.2-0.3 \mathrm{~s}$. The reason is that calculating the Pearson correlation coefficient is significantly more computationally expensive than comparing the slopes. The processing speed is still slow for real-time imaging however, this is a considerable advantage of the proposed method over the previous one [4].

\subsection{Conclusion}

In this work, we have shown the feasibility of identifying reflection artifacts (RAs) in PAI using 2-wavelength excitation. Results show that a reasonable RA identification can be achieved by using a proper combination of 2 wavelengths. The proposed method offers significant advantages over the previous method using 4 wavelengths [4] in terms of processing time and imaging system compactness and cost. 


\subsection{References}

[1] I. Steinberg, D. M. Huland, O. Vermesh, H. E. Frostig, W. S. Tummers, and S. S. Gambhir, "Photoacoustic clinical imaging," Photoacoustics 14, 77-98 (2019).

[2] S. M. Schoustra, D. Piras, R. Huijink, T. J. Op't Root, L. Alink, W. M. Kobold, W. Steenbergen, and S. Manohar, "Twente Photoacoustic Mammoscope 2: system overview and three-dimensional vascular network images in healthy breasts," J. Biomed. Opt. 24(12), 121909 (2019).

[3] S. Manohar, and M. Dantuma, "Current and future trends in photoacoustic breast imaging," Photoacoustics 16, 100134 (2019).

[4] H. N. Y. Nguyen, A. Hussain, and W. Steenbergen, "Reflection artifact identification in photoacoustic imaging using multi-wavelength excitation," Biomed. Opt. Express 9(10), 4613-4630 (2018).

[5] H. N. Y. Nguyen, and W. Steenbergen, "Reducing artifacts in photoacoustic imaging by using multi-wavelength excitation and transducer displacement," Biomed. Opt. Express 10(7), 3124-3138 (2019).

[6] M. K. A. Singh, and W. Steenbergen, "Photoacoustic-guided focused ultrasound (PAFUSion) for identifying reflection artifacts in photoacoustic imaging," Photoacoustics 3(4), 123-131 (2015).

[7] M. Jaeger, J. C. Bamber, and M. Frenz, "Clutter elimination for deep clinical optoacoustic imaging using localised vibration tagging (LOVIT)," Photoacoustics 1(2), 19-29 (2013).

[8] H.-M. Schwab, M. F. Beckmann, and G. Schmitz, "Photoacoustic clutter reduction by inversion of a linear scatter model using plane wave ultrasound measurements," Biomed. Opt. Express 7(4), 1468-1478 (2016).

[9] M. Jaeger, L. Siegenthaler, M. Kitz, and M. Frenz, "Reduction of background in optoacoustic image sequences obtained under tissue deformation," J. Biomed. Opt. 14(5), 054011 (2009). 
[10] D. Allman, A. Reiter, and M. A. L. Bell, "Photoacoustic source detection and reflection artifact removal enabled by deep learning," IEEE transactions on medical imaging 37(6), 1464-1477 (2018).

[11] A. Wiacek, K. C. Wang, H. Wu, and M. A. L. Bell, "Dual-wavelength photoacoustic imaging for guidance of hysterectomy procedures," in Advanced Biomedical and Clinical Diagnostic and Surgical Guidance Systems XVIII (International Society for Optics and Photonics, 2020), p. 112291D.

[12] J. Märk, H. Dortay, A. Wagener, E. Zhang, J. Buchmann, C. Grötzinger, T. Friedrich, and J. Laufer, "Dual-wavelength 3D photoacoustic imaging of mammalian cells using a photoswitchable phytochrome reporter protein," Communications Physics 1(1), 1-10 (2018).

[13] A. A. Leino, A. Pulkkinen, and T. Tarvainen, "ValoMC: a Monte Carlo software and MATLAB toolbox for simulating light transport in biological tissue," OSA Continuum 2(3), 957-972 (2019).

[14] S. L. Jacques, "Optical properties of biological tissues: a review," Phys. Med. Biol. 58(11), R37 (2013).

[15] H. Azhari, Basics of biomedical ultrasound for engineers (John Wiley \& Sons, 2010).

[16] J. L. Prince, and J. M. Links, Medical imaging signals and systems (Pearson Prentice Hall Upper Saddle River, 2006).

[17] T. Feng, Q. Li, C. Zhang, G. Xu, L. J. Guo, J. Yuan, and X. Wang, "Characterizing cellular morphology by photoacoustic spectrum analysis with an ultra-broadband optical ultrasonic detector," Opt. Express 24(17), 19853-19862 (2016).

[18] R. Michels, F. Foschum, and A. Kienle, "Optical properties of fat emulsions," Optics Express 16(8), 5907-5925 (2008).

[19] P. Beard, "Biomedical photoacoustic imaging," Interface Focus 1(4), 602-631 (2011).

[20] S. Hindel, A. Söhner, M. Maßß, W. Sauerwein, D. Möllmann, H. A. Baba, M. Kramer, and L. Lüdemann, "Validation of blood volume fraction quantification with 3D 
gradient echo dynamic contrast-enhanced magnetic resonance imaging in porcine skeletal muscle," PLOS One 12(1), e0170841 (2017). 



\section{Discussion and Outlook}

Current efforts focus on translating the photoacoustic imaging (PAI) technique to clinical applications. However, the occurrence of artifacts still remains a major drawback challenging the reliability of this imaging technique. This thesis proposed several methods for identifying and reducing in-plane and out-of-plane artifacts. The proposed methods still need further improvements to be clinically suitable.

\subsection{In-plane artifacts}

In PAI, the forward generated US waves can be reflected back due to the acoustic heterogeneity of the sample causing in-plane artifacts (IPAs), also called reflection artifacts. This thesis presented 2 methods for identifying these artifacts: using multi-wavelength excitation (at least 3 wavelengths) and using 2-wavelength excitation. These two methods exploit the wavelength dependence of local light fluence. However, within a small volume, the variation of the light fluence might be minor resulting in a region in which methods might misidentify real image features as IPAs. To avoid this, for each real image feature, the methods do not find any IPAs within a region below that image feature. Specific wavelengths might be used to minimize the size of that region. Additionally, the 2 methods might not be able to identify IPAs that appear at the same position with another image feature. A different approach such as PAFUSion [1, 2] might be needed. This method uses focused US to mimic PA sources reproducing IPAs. By subtracting the reproduced IPAs from the acquired PA signal, IPAs can be reduced. However, the main limitation of this method is that it only works when the acoustic reflector is in the image plane, and perpendicular to it so it is present in the US image.

Compared to pure PAI, these two methods require more post-processing thus reduce the frame rate. It was shown that these two methods can correct an image in $\sim 0.2 \mathrm{~s}$ with using segmentation in offline processing. However, the processing time still needs to be further reduced to make the methods clinically applicable, especially the approach without using segmentation. 
Both two methods (using multi-wavelength excitation and using 2-wavelength excitation) can identify IPAs well. However the way they reduce the identified artifacts (setting the corresponding pixels to 0) still needs further improvements, since the used method also erases the background, which might contain important information.

The demonstrations of the method using 2 wavelengths showed its capability of identifying and reducing IPAs, however, this method is strongly sensitive to noise. Additionally, choosing the 2 wavelengths is crucial as it was shown that a 2 -wavelength combination might not work properly in all cases. Nevertheless, in in vivo PAI, melanin and hemoglobin are the main chromophores and based on their well-known absorption spectrum, various 2-wavelength combinations can be used, specially wavelengths in the range of 800$900 \mathrm{~nm}$.

The method using 2-wavelength excitation is more favorable for clinical use as it requires fewer wavelengths resulting in a more compact and affordable PAI system compared to the one using multi-wavelength excitation. With 2 selected wavelengths for in vivo imaging as discussed above, this method still can provide reliable results. Moreover, using a 2-wavelength PAI system is a current research focus for clinical applications [3-5].

\subsection{Out-of-plane artifacts}

Out-of-plane artifacts (OPAs) originate from absorbers located outside the imaging plane. The methods for identifying IPAs cannot work for OPAs since they are real absorbers. This thesis proposed a method using axial transducer displacement to de-correlate in-plane image features and OPAs. The method was implemented experimentally with a compact PAI system showing its capability for clinical use. However, further improvements are still needed.

The main concern of this method is its capability of working in real-time as in the demonstration, it took $\sim 5$ minutes to correct OPAs in one image. This was mainly due to technical limitations such as a low repetition rate of the laser or a low speed of the translating stage. Additionally, the imaging object has to stay still in the whole process as slight movements would result in OPA mis-correction. This makes the method in its current form clinically inconvenient. However, using a high repetition rate laser and a fast translating stage would significantly reduce the processing time of the method. 
Similar to the methods for identifying IPAs, this method also reduces artifacts by erasing the corresponding pixels resulting in losing background information. A more natural approach to reduce the artifacts as in $[1,2]$ should be considered in the future development.

The method requires the imaging plane to stay the same during the displacement. In other words, the user has to displace the probe strictly in the axial direction. A probe holder that can automatically adjust the probe axially might be a solution and will be investigated in future work. Additionally, for clinical use, the translation stage must have a high speed enough that movement of the user can be neglected.

As the method has to track image features during the displacement, segmentation is needed. In the demonstration, a simple segmentation algorithm based on the Sobel edge detection was used for a proof of concept. However, segmentation does not always work properly resulting in OPA mis-correction. A more effective segmentation algorithm such as combing the Sobel edge detection with the watershed transform as in [6] should be considered to enhance the reliability of the method.

This method was further exploited later in the thesis showing that it can also provide a three-dimensional (3D) view of OPAs. The reconstructed pressure distribution image at each position of the probe during the axial displacement is back-projected elevationally forming a 3D projection. A 3D image is formed by using back-projection and multiplication for all the $3 \mathrm{D}$ projections. This $3 \mathrm{D}$ reconstruction method on one hand can reduce OPAs in the inplane image, on the other hand can reconstruct the out-of-plane absorbers.

Though this 3D reconstruction method can three-dimensionally visualize out-of-plane absorbers, it cannot reconstruct in-plane absorbers in an elevationally correct manner. All reconstructed in-plane absorbers appear in a curved shape in the elevation direction. This is a reconstruction artifact due to a limited number of projections. An additional method to reduce the streak-type reconstruction artifacts such as [7] can be used to overcome this.

In addition to the time needed for displacing the transducer array, the 3D reconstruction is also computationally expensive. It is still far from real-time imaging. As discussed above, using a high repetition rate laser and a fast translating stage would be a solution to significantly reduce the performance time. 


\subsection{References}

[1] M. K. A. Singh, M. Jaeger, M. Frenz, and W. Steenbergen, "Photoacoustic reflection artifact reduction using photoacoustic-guided focused ultrasound: comparison between plane-wave and element-by-element synthetic backpropagation approach," Biomed. Opt. Express 8(4), 2245-2260 (2017).

[2] M. K. A. Singh, and W. Steenbergen, "Photoacoustic-guided focused ultrasound (PAFUSion) for identifying reflection artifacts in photoacoustic imaging," Photoacoustics 3(4), 123-131 (2015).

[3] S. M. Schoustra, D. Piras, R. Huijink, T. J. op't Root, L. Alink, W. M. Kobold, W. Steenbergen, and S. Manohar, "Twente Photoacoustic Mammoscope 2: system overview and three-dimensional vascular network images in healthy breasts," J. Biomed. Opt 24(12), 121909 (2019).

[4] J. Märk, H. Dortay, A. Wagener, E. Zhang, J. Buchmann, C. Grötzinger, T. Friedrich, and J. Laufer, "Dual-wavelength 3D photoacoustic imaging of mammalian cells using a photoswitchable phytochrome reporter protein," Commun. Phys. 1(1), 1-10 (2018).

[5] A. Wiacek, K. C. Wang, H. Wu, and M. A. L. Bell, "Dual-wavelength photoacoustic imaging for guidance of hysterectomy procedures," in Advanced Biomedical and Clinical Diagnostic and Surgical Guidance Systems XVIII (International Society for Optics and Photonics, 2020), p. 112291D.

[6] H. Ng, S. Ong, K. Foong, P. Goh, and W. Nowinski, "Medical image segmentation using k-means clustering and improved watershed algorithm," in 2006 IEEE southwest symposium on image analysis and interpretation (IEEE, 2006), pp. 61-65.

[7] C. Cai, X. Wang, K. Si, J. Qian, J. Luo, and C. Ma, "Streak artifact suppression in photoacoustic computed tomography using adaptive back projection," Biomed. Opt. Express 10(9), 4803-4814 (2019). 


\section{Summary}

Throughout the thesis, it is shown how artifacts negatively affect the reliability of the photoacoustic imaging technique. This thesis focuses on in-plane and out-of-plane artifacts. Several approaches are proposed in this thesis in order to identify and reduce these artifacts. This section will summarize all the work reported in this thesis.

\subsection{Chapter 2}

The second chapter of this thesis highlights the state-of-art applications and translational efforts of PAI over the past few years. As PAI provides localized information at ultrasound imaging depth, endogenous chromophores and contrast agents can be used to enhanced the image contrast. Of the endogenous chromophores, hemoglobin is a main target in PAI as the information of this chromophore might reveal angiogenesis or hypoxia which are hallmarks of the lesions. This has been demonstrated in numerous preclinical studies on various human diseases: breast cancer, Crohn's disease, rheumatoid arthritis, psoriasis, prostate cancer, atherosclerotic plaque. The PA image contrast can be further enhanced by using exogenous contrast agents. Additionally, activated contrast agents turn into gas bubbles increasing ultrasound image contrast. Recent research also takes advantage of the contrast agents in photothermal therapy as in tumor treatment with localized thermal ablation. The remaining barriers and future suggestion to bring PAI closer to clinical application are discussed at the end of this chapter.

\subsection{Chapter 3}

This chapter proposes a method for identifying and reducing reflection artifacts (RAs), also called in-plane artifacts, using multi-wavelength excitation. This method analyzes the spectral response of the image features obtained by imaging the sample with multiple wavelengths. Particularly, 4 wavelengths $(808,915,940$ and $980 \mathrm{~nm})$ were used in this work.

It is assumed that: (1) Absorbers with identical optical properties located at different positions give different spectral responses due to different wavelength dependence of the local light fluence, (2) Both RAs and their real image feature have a similar spectral response 
representing the optical properties of the absorber and the wavelength dependent optical fluence at the absorber location. With these 2 assumptions, RAs then have a similar spectral response as their real image feature. The similarity between the spectral responses is quantified by using the Pearson correlation coefficient. Additionally, RAs have weaker signals and appear at a larger depth than their real image feature as a consequence of longer ultrasound wave propagation. With these findings, RAs can be identified.

The method was first validated with a phantom experiment in which 2 identical absorbers were placed at different depths. An acoustic reflector was placed underneath the upper absorber such as to get RAs at the depth of the lower absorber. Results showed that the method is able to identify the RAs without misidentifying the lower absorber as an RA. These results proved the 2 assumptions. The method was then also validated in vivo where a finger was imaged. The in vivo image contained RAs of the skin and blood vessels signals reflected off the bone layer. The method again could identify all the RAs.

With the method experimentally validated, limitations of assumption (1) were then verified by imaging 2 identical absorbers placed at different depths in a tissue mimicking medium. The depth distance $\Delta z$ between the 2 absorbers was adjusted to find out the value of $\Delta z_{\text {min }}$ for which local light fluence is different enough to distinguish the 2 absorbers, such avoiding the deeper absorber to be misidentified as an RA of the other one. The studied showed that with the 4 wavelengths used in this work, a value of $\Delta z_{\min }=1.7 \mathrm{~mm}$ applies. In other words, the method only can identify RAs at least $1.7 \mathrm{~mm}$ underneath an absorber.

In order to detect image features, a segmentation algorithm based on the Sobel edge detection was used. However, the method was also implemented with a non-segmentation approach. In this approach, all pixels in the acquired image were compared to each other for the spectral responses. The results of this approach were in a great agreement with the approach using segmentation.

The experiments in this work were carried out using a compact PAI system showing the potential of this method for correcting RAs in real-time and the practical applicability of this method for medical use. 


\subsection{Chapter 4}

This chapter proposes a new method for identifying and reducing out-of-plane artifacts (OPAs). It demonstrates again the method identifying in-plane artifacts (IPAs), also called reflection artifacts, using the multi-wavelength excitation presented in Chapter 3 . The two methods are then combined together to identify and reduce both IPAs and OPAs.

The principle of the method for identifying OPAs is based on the different behaviors of in-plane image features and OPAs when displacing the transducer array axially. Particularly, when axially displacing up the transducer array, in-plane image features move down exactly the same distance as the displacement while OPAs travel a lesser extent. By exploiting these different behaviors, OPAs can then be distinguished from in-plane image features.

A phantom producing both IPAs and OPAs was used to validate the method. The outof-plane absorber placed $\sim 3-4 \mathrm{~mm}$ outside of the imaging plane elevationally was still identified as an OPA with $5 \mathrm{~mm}$ axial displacement of the transducer array. After removing the OPAs, IPAs were also processed with 8 images using 8 excitation wavelengths using the method described in Chapter 3. In the final corrected images when both methods were applied, all IPAs and OPAs were removed.

Another phantom mimicking an OPA overlapping an in-plane feature image was also used to verify whether the proposed method could remove the OPA and keep the in-plane image feature. The experimental results not only successfully addressed the concern but also showed that the method could also potentially reduce the streak-type reconstruction artifacts.

The method was then validated in in vivo imaging. A finger with a black ink mark on the skin was imaged giving an image with clear IPAs and OPAs. The same steps as in the first phantom experiment were carried out. In the final corrected image, IPAs and OPAs were again completely removed enhancing the capability of the method.

All experimental work was performed using a handheld US probe showing the potential of the proposed method for clinical use.

\subsection{Chapter 5}

The axial displacement of the transducer array method presented in Chapter 4 is further exploited in this chapter. Though the imaging plane remains the same during the axial 
displacement, this method still can provide a three-dimensional (3D) view of the OPAs. This chapter proposes a 3D reconstruction method using axial displacement.

At each position of the probe during the axial displacement, a reconstructed pressure distribution image (before the envelope detection) is back-projected in the axial-elevation plane forming a $3 \mathrm{D}$ projection. All 3D projections during the displacement are then combined, providing a 3D image of the sample.

Due to a limited total displacement distance and a limited number of displacement positions resulting in a limited number of $3 \mathrm{D}$ projections, the reconstructed $3 \mathrm{D}$ image has strong streak-type artifacts in the axial-elevation plane. Back projection and multiplication (BPM) which is in principle similar to the delay-multiply-and-sum reconstruction algorithm was used instead of the conventional direct back projection (BP) algorithm. BPM provides better 3D reconstruction with streak-type artifacts strongly reduced compared to BP, however, at a cost of more expensive computation.

The 3D reconstruction using axial displacement was explored in terms of axial, lateral, and elevation resolutions of an in-plane absorber and an out-of-plane absorber. It was also compared with the $3 \mathrm{D}$ reconstruction using elevation displacement. Results showed that elevationally displacing the probe forms a 3D image with more elevation information than the reconstruction using axial displacement. However, the quality of the in-plane image is significantly worse.

The proposed reconstruction method was then experimentally validated in phantoms and in vivo. $3 \mathrm{D}$ reconstructed images could visualize out-of-plane absorbers located $\sim 4 \mathrm{~mm}$ away from the imaging plane in both phantoms and in vivo with a total axial displacement distance of $2.625 \mathrm{~mm}$.

The in-plane image of the $3 \mathrm{D}$ reconstructed image was compared with the conventional reconstructed 2D image for the OPAs. The results showed that OPAs are strongly reduced in the in-plane image of the 3D image. Additionally, it was also compared with the reducing OPAs method presented in Chapter 4. While reducing OPAs with 3D reconstruction does not affect in-plane image features, it cannot remove completely OPAs as in the previous method. 
The sensitivity of the transducer array plays an important role in reconstructing images. Thus, compensating the sensitivity might improve the reliability of the reconstructed image. In this work, the sensitivity of the transducer array was experimentally measured and taken into account in the 3D reconstruction. Signals of out-of-plane absorbers were significantly more pronounced in the sensitivity compensated 3D. This is reasonable as the light fluence at the out-of-plane absorbers was much higher than inside the imaging plane.

In vivo demonstrations of the proposed $3 \mathrm{D}$ reconstruction method using a compact PAI system with a small axial displacement distance $(2.625 \mathrm{~mm})$ show promising clinical capability of this method, once this displacement can be applied automatically and sufficiently fast.

\subsection{Chapter 6}

The method for identifying and reducing RAs using multi-wavelength excitation presented in Chapter 3 showed its potential for clinical applications. However, that method requires at least 3 wavelengths. For the affordability of the method, the number of wavelengths should be as small as possible. This chapter simplifies the method by reducing the number of wavelengths to 2 while still preserving advantages of the method.

In this method, the 2-wavelength response of the RAs is also assumed to be similar to the one of the real image feature. Instead of using the Pearson correlation coefficient to quantify the similarity between the responses as in the previous method which requires at least 3 wavelengths, the slope of the 2-wavelength is used in this method. The 2-wavelength responses, obtained in the same way as obtaining spectral responses reported in Chapter 3, are compensated for the output energy of the corresponding wavelength and then normalized to their maximum. The slope of each 2-wavelength response is determined by subtracting the first from the second wavelength response. All the slopes in the image are subtracted from each other. The differences are compared with a threshold to separate small values revealing similar slopes. The image features of these slopes are then compared in terms of signal amplitude and depth as RAs appear at a larger depth with a weaker signal than their real image features. Using this combination of properties, RAs can be identified.

The assumption that an RA and its real image feature have similar slopes was first validated with a simulation as a theoretical backing. The validation was then performed in 
phantom and in vivo showing the feasibility of this method for identifying RAs. The method was also validated with the approach without using segmentation. The results again showed the potential of the method for identifying RAs and were in good agreement with the previous method using 4 wavelengths.

The selection of the 2 wavelengths is crucial to achieve a reasonable result as the experimental results indicated that a 2-wavelength combination does not work properly in all cases. Prior knowledge of the sample is required. However, in vivo imaging, the main chromophores are melanin and hemoglobin and their optical absorption spectra are wellknown. 2 wavelengths at $800 \mathrm{~nm}$ and another one in between 810-900 nm are an excellent option.

As the 2-wavelength response is strongly sensitive to noise, the robustness of the method was analyzed in a range of signal-to-noise ratios (SNRs). The analysis showed that an SNR of $\sim 5.2 \mathrm{~dB}$ and lower, the method tends to misidentify real image features as RAs.

In addition to having all main advantages as the method using 4 wavelengths, the method using 2 wavelengths outperforms the previous method in terms of processing time, imaging system cost and compactness. 


\section{Samenvatting}

In dit proefschrift is aangetoond hoe artefacten de betrouwbaarheid van fotoakoestische beeldvorming negatief kunnen beïnvloeden. Dit proefschrift focust zich op in-plane en outof-plane artefacten tijdens de beeldvorming. Meerdere methoden worden gepresenteerd om deze artefacten te identificeren en te verwijderen. Deze paragraaf vat al het werk samen dat terug te vinden is in dit proefschrift.

\subsection{Hoofdstuk 2}

Het tweede hoofdstuk focust zich op de nieuwste toepassingen en translationele inspanningen van fotoakoestiek van de laatste paar jaren. Endogene chromoforen en contrastmiddelen kunnen gebruikt worden om het fotoakoestische contrast te verhogen, doordat fotoakoestiek gelokaliseerde informatie kan verstrekken op beelddieptes vergelijkbaar met ultrasound imaging. Van alle endogene chromoforen is hemoglobine de meest interessante, omdat deze chromofoor angiogenese of hypoxie kan onthullen, welke kenmerken zijn van laesies. Dit is aangetoond in een aantal preklinische studies, op verschillende ziektebeelden: borstkanker, de ziekte van Crohn, reumatoïde artritis, psoriasis, prostaatkanker en atherosclerotische plaques. Het fotoakoestische contrast kan verder versterkt worden door gebruik te maken van exogene contrastmiddelen. Daarnaast is het mogelijk om contrastmiddelen om te zetten in bubbels die het ultrasound contrast verhogen. Recent onderzoek naar fotothermische therapie maakt ook gebruik van contrastmiddelen voor de behandeling van tumoren met gelokaliseerde thermische ablatie. Aan het eind van dit hoofdstuk worden de openstaande uitdagingen en aanbevelingen om fotoakoestische beeldvorming naar de kliniek te brengen besproken.

\subsection{Hoofdstuk 3}

Dit hoofdstuk bespreekt een nieuwe methode om reflectieartefacten (RAs), ook wel inplane artefacten genoemd, te reduceren door gebruik te maken van meerdere optische golflengtes en door vervolgens de spectrale reactie van de absorberende objecten te 
analyseren. Er wordt voornamelijk gebruik gemaakt van vier golflengtes (808, 915, 940 en $980 \mathrm{~nm})$.

Er wordt aangenomen dat: (1) absorberende objecten die zich op verschillende locaties bevinden maar identieke optische eigenschappen hebben, spectraal verschillend reageren door de verschillen in lokale licht fluence, (2) zowel de echte bronnen als hun RAs vergelijkbare spectrale reacties hebben, die bepaald worden door de optische eigenschappen van het absorberende object en de golflengte-afhankelijke optische fluence op de locatie van het object. Gebaseerd op deze twee aannames kunnen we zeggen dat de RAs en de echte objecten een vergelijkbare spectrale reactie hebben. De overeenkomst tussen de spectrale reacties wordt gekwantificeerd door gebruik te maken van de Pearson correlatiecoëfficiënt. Daarnaast hebben RAs zwakkere signalen en bevinden ze zich op grotere beelddieptes dan het signaal van de echte bron, door een langere ultrasound reistijd. Met deze methodes zijn we in staat om de RAs te identificeren.

De methode is gevalideerd met behulp van een fantoomexperiment, waarin twee identieke absorberende objecten op verschillende dieptes geplaatst waren. Een akoestische reflector was onder het bovenste absorberende object geplaatst, zodat RAs verkregen zouden worden op dezelfde diepte als het lagere object. Resultaten lieten zien dat de methode in staat is om de RAs te identificeren zonder het onderste object aan te zien voor een RA. Deze resultaten hebben de twee aannames bevestigd. De methode is vervolgens ook in vivo gevalideerd, met behulp van een meting op een vinger. Het in vivo beeld bevatte RAs afkomstig van de fotoakoestische signalen van de huid en bloedvaten die reflecteren op het bot. De methode was ook hier in staat om alle RAs te identificeren.

$\mathrm{Na}$ de experimentele validatie zijn de limitaties van aanname (1) geverifieerd door twee identieke absorberende objecten op verschillende dieptes in een weefsel-nabootsend medium te plaatsen. De afstand $\Delta z$ tussen de twee objecten is gevarieerd, om zo de minimale afstand $\Delta z_{\min }$ te vinden waar de licht fluence zodanig verschillend is dat de twee objecten geïdentificeerd kunnen worden, maar zodanig dat het diepere object niet aangezien werd als een RA van het andere object. De studie heeft laten zien dat de vier golflengtes die gebruikt zijn, leiden tot een $\Delta z_{\min }=1.7 \mathrm{~mm}$. Met andere woorden, de methode is alleen in staat om RAs te identificeren die zich ten minste $1.7 \mathrm{~mm}$ onder het object bevinden. 
Om objecten in de afbeelding te detecteren is een segmentatiealgoritme gebaseerd op de Sobel rand detectie gebruikt. De methode is ook getest zonder segmentatie. Voor deze benadering zijn alle spectrale reacties van de pixels in de verkregen afbeelding met elkaar vergeleken. De resultaten van deze benadering kwamen sterk overeen met de resultaten van de benadering met de segmentatie.

De experimenten in dit werk zijn uitgevoerd met een compact fotoakoestieksysteem, die de potentie van deze methode om real-time te corrigeren voor RAs en de praktische toepasbaarheid van deze methode in de klinische omgeving laat zien.

\subsection{Hoofdstuk 4}

Dit hoofdstuk stelt een nieuwe methode voor om out-of-plane artefacten (OPAs) te identificeren en verminderen. Het demonstreert opnieuw de methode om in-plane artefacten (IPAs) te identificeren, ook wel reflectieartefacten (RAs) genoemd, gebruikmakend van de methode met excitatie met meerdere golflengtes, gepresenteerd in Hoofdstuk 3. Beide methodes worden gecombineerd om zowel IPAs als OPAs te identificeren en reduceren.

Het principe van de methode voor het identificeren van OPAs is gebaseerd op het verschil in gedrag van in-plane beeldkenmerken en OPAs wanneer de transducer axiaal getransleerd wordt. Wanneer de transducer axiaal omhoog getransleerd wordt, zullen inplane beeldkenmerken precies dezelfde afstand naar beneden bewegen als deze translatie. OPAs zullen minder bewegen. Door het exploiteren van dit verschil in gedrag, kunnen OPAs onderscheiden worden van in-plane beeldkenmerken.

Een fantoom wat zowel IPAs als OPAs produceerde is gebruikt om de methode te valideren. Het out-of-plane absorberende object, geplaatst op 3-4 mm buiten het beeldvlak in de elevational richting, werd nog geïdentificeerd als een OPA met een $5 \mathrm{~mm}$ axiale translatie van de transducer. Na het verwijderen van de OPAs, zijn ook de IPAs verwerkt met acht afbeeldingen en met het gebruik van acht excitatiegolflengtes, volgens de methode beschreven in Hoofdstuk 3. In de definitieve gecorrigeerde afbeeldingen, met toepassing van beide methodes, waren alle IPAs en OPAs verwijderd.

Een ander fantoom bootst een OPA na die overlapt met een in-plane kenmerk. Dit fantoom is gebruikt om te verifiëren of de voorgestelde methode de OPA kon verwijderen 
met behoud van het in-plane beeldkenmerk. De experimentele resultaten lieten niet alleen een succesvolle aanpak van dit probleem zien, maar demonstreerden ook dat de methode streepachtige reconstructieartefacten zou kunnen reduceren.

De methode is vervolgens gevalideerd in in vivo beeldvorming. Een vinger, met een markering van zwarte inkt op de huid, is afgebeeld en het resultaat toonde duidelijk IPAs en OPAs. Dezelfde stappen als in het eerste fantoomexperiment zijn genomen. In de definitieve gecorrigeerde afbeelding waren wederom alle IPAs en OPAs verwijderd, wat de geschiktheid van de methode verbetert.

$\mathrm{Al}$ het experimentele werk is gedaan met een ultrasound probe welke in de hand gehouden kan worden, wat het potentieel van deze methode voor klinisch gebruik laat zien.

\subsection{Hoofdstuk 5}

De methode van axiale verplaatsing van de transducer array zoals gepresenteerd in Hoofdstuk 4 wordt verder gebruikt in dit hoofdstuk. Hoewel het beeldvlak hetzelfde blijft tijdens axiale verplaatsing, kan deze methode nog steeds een driedimensionaal (3D) beeld van de OPAs geven. Dit hoofdstuk stelt een 3D reconstructiemethode voor waarbij de axiale verplaatsing gebruikt wordt.

Op iedere positie van de probe tijdens de axiale verplaatsing wordt een gereconstrueerde afbeelding van de drukverdeling (voor detectie van de omhullende) terug geprojecteerd in het axiale-elevation vlak, waardoor een 3D projectie gevormd wordt. Alle 3D projecties tijdens de verplaatsing worden gecombineerd, wat zorgt voor een $3 \mathrm{D}$ afbeelding van het monster.

Vanwege een gelimiteerde maximale verplaatsing en een gelimiteerd aantal verplaatsingsposities, resulterend in een gelimiteerd aantal 3D projecties, heeft het gereconstrueerde 3D beeld sterke streepachtige artefacten in het axiale-elevation vlak. Terug projectie en vermenigvuldiging (back projection and multiplication, BPM), wat in principe vergelijkbaar is met het vertraag-vermenigvuldig-en-sommeer (delay-multiply-and-sum) reconstructiealgoritme, is gebruikt in plaats van het conventionele directe terug projectie (back projection, BP) algoritme. BPM leidt tot een betere 3D reconstructie met sterk 
verminderde streepachtige artefacten in vergelijking met BP, maar dit gaat wel ten koste van rekensnelheid.

De $3 \mathrm{D}$ reconstructie gebruikmakend van axiale verplaatsing is verkend in termen van axiale, laterale en elevation resoluties van een in-plane en een out-of-plane absorberend object. Het is daarnaast vergeleken met de 3D reconstructie die gebruik maakt van elevation verplaatsing. Resultaten laten zien dat het elevationally verplaatsen van de probe een $3 \mathrm{D}$ afbeelding geeft met meer elevation informatie dan de reconstructie die de axiale verplaatsing gebruikt. Echter, de kwaliteit van de in-plane afbeelding is aanzienlijk slechter.

De voorgestelde reconstructiemethode is toen experimenteel gevalideerd in fantomen en in vivo. 3D gereconstrueerde afbeeldingen konden out-of-plane absorberende objecten visualiseren die op $\sim 4 \mathrm{~mm}$ afstand van het beeldvlak gelokaliseerd waren, zowel in fantomen als in vivo, met een totale axiale verplaatsing van $2.625 \mathrm{~mm}$.

De in-plane afbeelding van de 3D gereconstrueerde afbeelding is vergeleken met de conventionele gereconstrueerde 2D afbeelding voor de OPAs. De resultaten laten zien dat OPAs sterk gereduceerd worden in de in-plane afbeelding van de 3D afbeelding. Daarnaast is het ook vergeleken met de in Hoofdstuk 4 gepresenteerde methode voor het reduceren van OPAs. Terwijl het reduceren van OPAs met een 3D reconstructie niet de in-plane beeldkenmerken beïnvloedt, kan het niet de OPAs helemaal verwijderen zoals in de vorige methode.

De gevoeligheid van de transducer array speelt een belangrijke rol in het reconstrueren van afbeeldingen. Dus, het compenseren van de gevoeligheid zou de betrouwbaarheid van de gereconstrueerde afbeelding kunnen verbeteren. In dit werk is de gevoeligheid van de transducer array gemeten en is er rekening mee gehouden in de 3D reconstructie. Signalen van out-of-plane absorberende objecten waren aanzienlijk meer uitgesproken in de 3D methode met gecompenseerde gevoeligheid. Dit is aannemelijk, omdat de licht fluence bij de out-of-plane absorberende objecten veel hoger was dan binnen het beeldvlak.

In vivo demonstraties van de voorgestelde 3D reconstructiemethode, gebruikmakend van een compact fotoakoestieksysteem met een kleine axiale verplaatsing $(2.625 \mathrm{~mm})$, laten veelbelovend klinisch potentieel zien, zodra deze verplaatsing automatisch en snel genoeg toegepast kan worden. 


\subsection{Hoofdstuk 6}

De methode gepresenteerd in Hoofdstuk 3, om RAs te identificeren en onderdrukken door gebruik te maken van excitatie met meerdere golflengtes, heeft zijn potentie voor klinische toepassingen laten zien. Echter, deze methode heeft op zijn minst drie excitatiegolflengtes nodig. Om de methode betaalbaar te laten zijn, moet het aantal golflengtes geminimaliseerd worden. In dit hoofdstuk versimpelen we de methode, door het aantal golflengtes terug te brengen naar twee, terwijl de voordelen van de methode behouden worden.

In deze methode wordt er aangenomen dat de 2-golflengte reactie van de RAs hetzelfde is als die van een echt absorberend object. In plaats van gebruik te maken van de Pearson correlatiecoëfficiënt om de overeenkomsten tussen de reacties te kwantificeren, zoals gedaan is in de vorige methode, wordt nu de helling van de 2-golflengte reactie gebruikt. De 2golflengte reactie, verkregen op dezelfde manier als in Hoofdstuk 3, wordt gecompenseerd voor de outputenergie voor de betreffende golflengtes en vervolgens genormaliseerd naar hun maximum. De helling van de 2-golflengte reactie wordt bepaald door eerst de amplitude van de eerste golflengte van die van de tweede af te trekken. Alle hellingen in de afbeelding worden van elkaar afgetrokken. De verschillen worden dan vergeleken met een threshold, om zodoende kleine waardes die verglijkbare hellingen laten zien te selecteren. De beeldkarakteristieken van deze hellingen worden dan vergeleken in termen van signaalamplitude en diepte, omdat RAs voorkomen op grotere dieptes met een zwakkere amplitude dan de echte objecten. Gebruikmakend van deze combinatie van eigenschappen kunnen de RAs geïdentificeerd worden.

De aanname dat een RA en zijn oorspronkelijke object dezelfde helling hebben is eerst gevalideerd met een simulatiestudie. De validatie is vervolgens ook met fantoommetingen en een in vivo studie gedaan. De methode is ook gevalideerd met de eerder beschreven aanpak zonder segmentatie. De resultaten van deze 2-golflengte methode kwamen goed overeen met de eerder gepresenteerde 4-golflengte methode.

De selectie van de twee golflengtes is cruciaal voor het verkrijgen van een goed resultaat. Experimenten toonden aan dat twee golflengtes niet altijd genoeg zijn, als er geen voorkennis over het monster beschikbaar is. Echter, in in vivo studies zijn de absorptiespectra 
van de chromoforen die aanwezig zijn bekend. De studie toonde aan dat een golflengte rond $800 \mathrm{~nm}$ en de andere tussen $810-900 \mathrm{~nm}$ goede opties zijn.

De robuustheid van de methode is onderzocht voor een aantal signaal-ruisverhoudingen (SNRs), omdat de 2-golflengte aanpak sterk afhankelijk bleek te zijn van ruis. De analyse toonde aan dat de methode fouten begint te maken voor een SNR van $\sim 5.2 \mathrm{~dB}$ en lager. Bij deze SNRs worden echte objecten aangezien voor RAs.

De 2-golflengte methode scoort beter dan de 4-golflengte methode op het gebied van verwerkingstijd, kosten en compactheid van het systeem. 



\section{Appendix}

\section{Appendix 1. Supplementary figures}

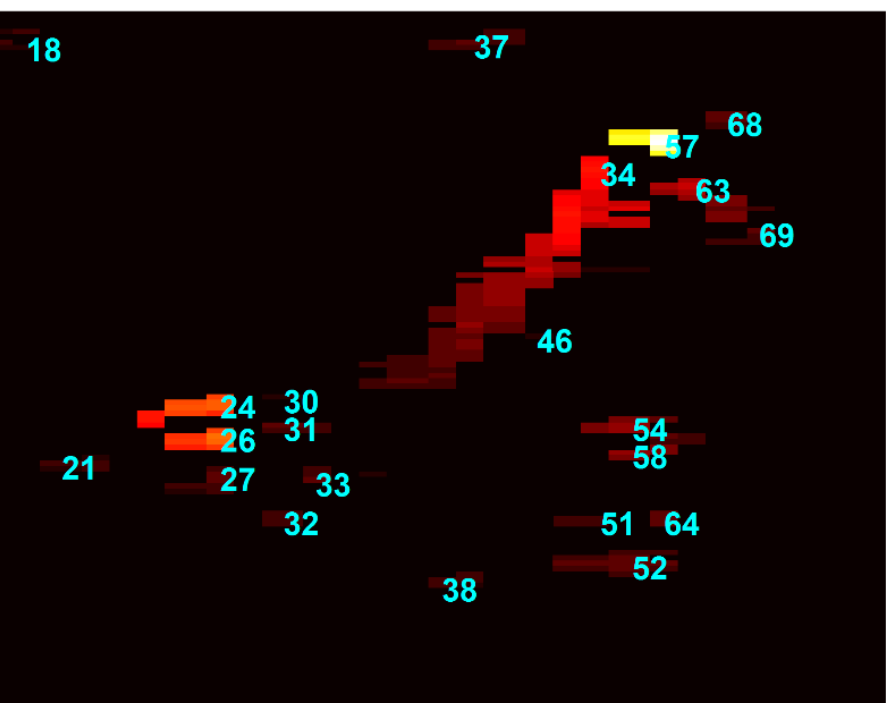

Appendix 1.1. Segmented image of the phantom with all features numbered [Fig. 3.7]. 


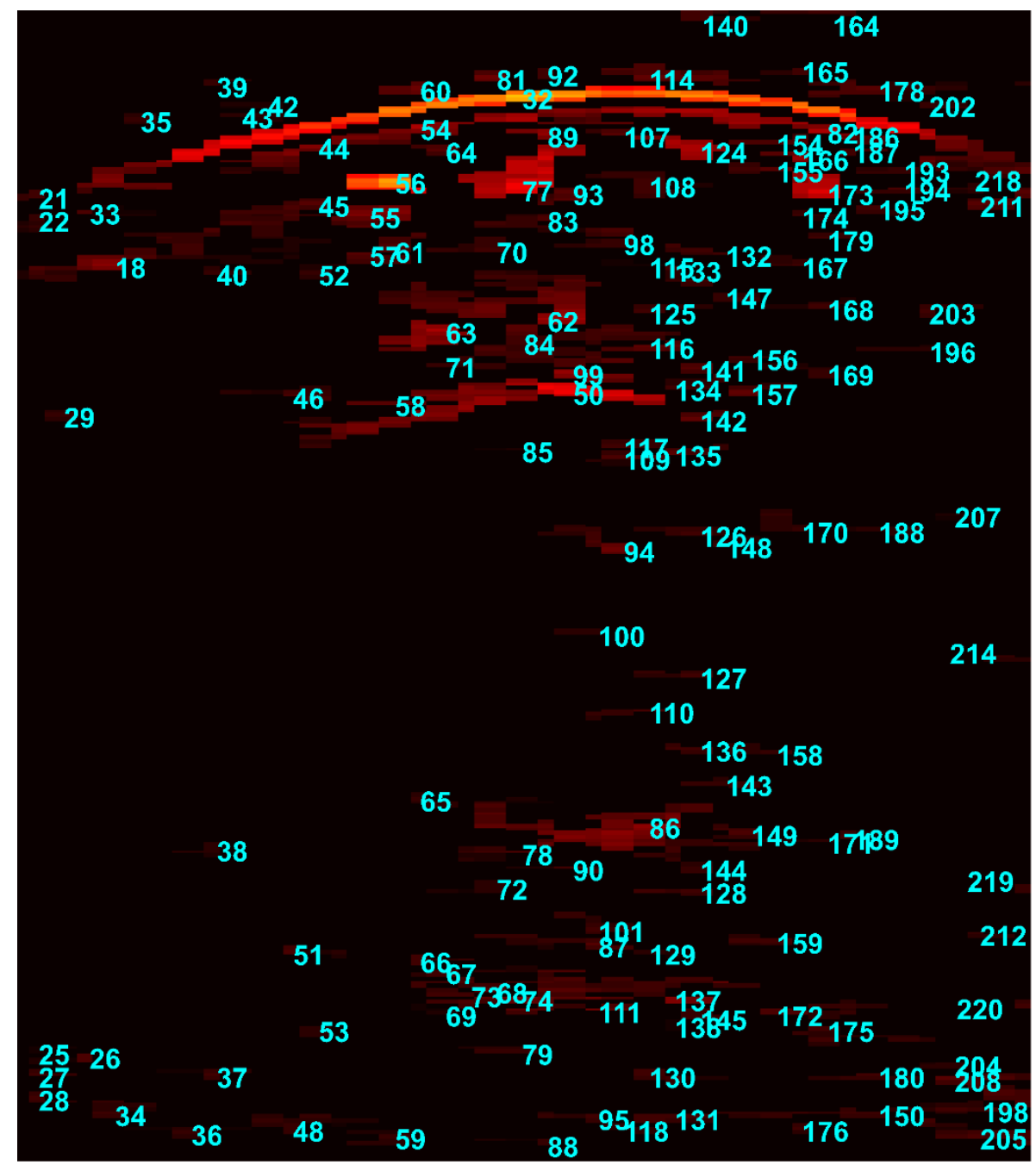

Appendix 1.2. Segmented in vivo image with all features numbered [Fig. 3.10]. 

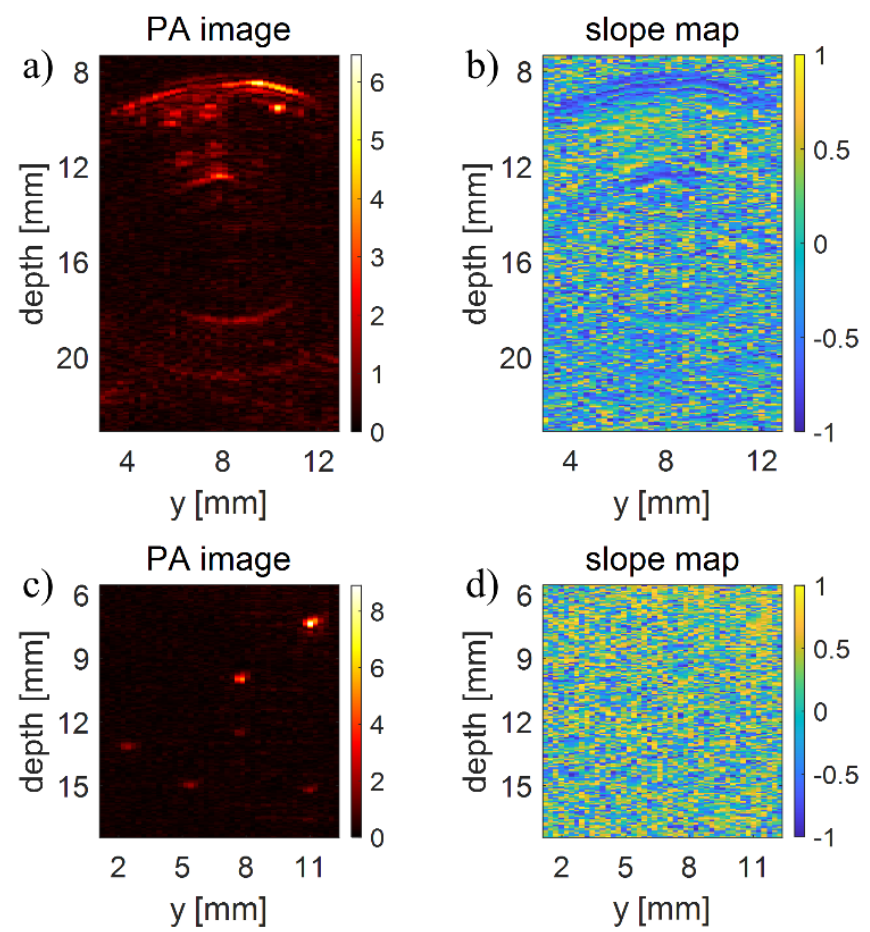

Appendix 1.3. PA images, (a) and (b), and the slope map, (c) and (d), of in vivo 2 and the phantom respectively [Fig. 6.10]. 


\section{Appendix 2. Links to supplementary data/videos}

Each Appendix line includes both links from the journal server (Link 1) and UTwente server (Link 2).

Appendix 2.1. Link 1 or Link 2

Appendix 2.2. Link 1 or Link 2

Appendix 2.3. Link 1 or Link 2

Appendix 2.4. Link 1 or Link 2

Appendix 2.5. Link 1 or Link 2

Appendix 2.6. Link 1 or Link 2

Appendix 2.7. Link 1 or Link 2

Appendix 2.8. Link 1 or Link 2

Appendix 2.9. Link 1 or Link 2

Appendix 2.10. Link 1 or Link 2

Appendix 2.11. Link 1 or Link 2

Appendix 2.12. Link 1 or Link 2

Appendix 2.13. Link 1 or Link 2

Appendix 2.14. Link 1 or Link 2

Appendix 2.15. Link 1 or Link 2

Appendix 2.16. Link 1 or Link 2

Appendix 2.17. Link 1 or Link 2

Appendix 2.18. Link 1 or Link 2

Appendix 2.19. Link 1 or Link 2

Appendix 2.20. Link 1 or Link 2 


\section{About the author}

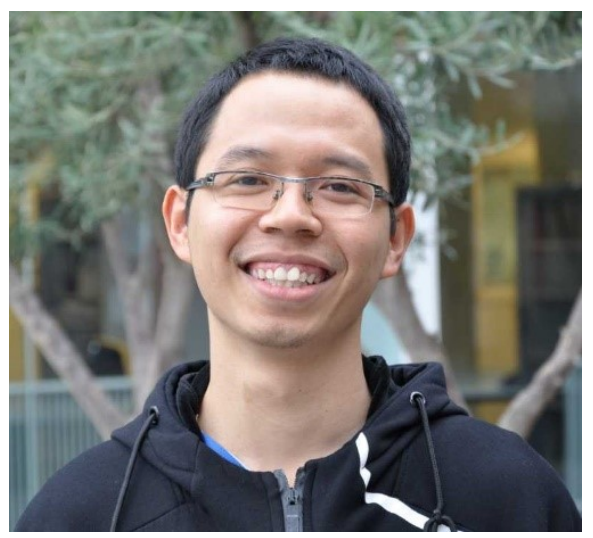

Ho Nhu Y Nguyen was born in April 1990

in Nghe An, Vietnam. He studied for a Bachelor of Engineer in Electronics and Telecommunications at the Hanoi University of Science and Technology from 2008 to 2013, in Hanoi. In the following year, he worked as a software developer at Samsung Vietnam R\&D Mobile Center in Hanoi. In 2014, he started following a Master of Science in Optics and Photonics as an Erasmus Mundus

Scholarship fellow. He studied at Aix-Marseille University, France in the first semester and at the Karlsruhe Institute of Technology, Germany in the second and third semesters. $\mathrm{He}$ went to Munich, Germany for his Master thesis where he worked on developing a dualwavelength hybrid photoacoustic-ultrasound microscope at the Institute of Biological and Medical Imaging, Helmholtz Zentrum Muenchen. He continued working here as a research assistant for 3 months after his graduation.

In February, 2017, he started pursuing a PhD degree at the Biomedical Photonic Imaging group, University of Twente, The Netherlands under supervision of Prof. dr. ir. Wiendelt Steenbergen. His research was part of a European Union's Horizon 2020 research and innovation program, and focused on identifying and reducing in-plane and out-of-plane artifacts in photoacoustic imaging. 


\section{Publications}

\section{Peer reviewed articles}

J. Yu*, H. N. Y. Nguyen*, W. Steenbergen, and K. Kim, "Recent development of technology and application of photoacoustic molecular imaging toward clinical translation," Journal of Nuclear Medicine 59(8), 1202-1207 (2018).

H. N. Y. Nguyen, A. Hussain, and W. Steenbergen, "Reflection artifact identification in photoacoustic imaging using multi-wavelength excitation," Biomedical Optics Express 9(10), 4613-4630 (2018).

H. N. Y. Nguyen, and W. Steenbergen, "Reducing artifacts in photoacoustic imaging by using multi-wavelength excitation and transducer displacement," Biomedical Optics Express 10(7), 3124-3138 (2019).

H. N. Y. Nguyen, and W. Steenbergen, "Three-dimensional view of out-of-plane artifacts in photoacoustic imaging using a laser-integrated linear-transducer-array probe," Photoacoustics, 100176 (2019).

H. N. Y. Nguyen, and W. Steenbergen, "Feasibility of identifying reflection artifacts in photoacoustic imaging using two-wavelength excitation," Biomedical Optics Express, 11(10), 5745-5759 (2020).

*Contributed equally to this work

\section{Conference Proceedings}

H. N. Y. Nguyen, and W. Steenbergen, "Out-of-plane artifact removal in photoacoustic imaging using transducer array displacement," in European Conference on Biomedical Optics (2019). 


\section{Conference presentations (oral and poster)}

H. N. Y. Nguyen, A. Hussain, and W. Steenbergen, "Reflection artifact reduction in photoacoustic imaging using multiple wavelengths" Photonics West: BiOS, San Francisco, USA (2018).

H. N. Y. Nguyen, and W. Steenbergen, "Reflection artifact identification in photoacoustic imaging using 2-wavelength excitation" Photonics West: BiOS, San Francisco, USA (2021) (accepted). 



\section{Acknowledgements}

It has been four memorable years working at the Biomedical Photonic Imaging group. To this end, I have received lots of help, support and encouragement from the people around me making my 4-year journey smoother. In this final section, I would like to express my sincere gratitude to them.

I would like to give the first words to my supervisor, Prof. Wiendelt Steenbergen. Thank you for giving me the opportunity to work on this project and guiding me through it. I really learned from you how to look into the problems from different angles and think deeper beyond the solutions. I also gained huge confidence in writing thanks to your guidance. I still remember how much worried I was in our first articles. I appreciate the working atmosphere you offered where I could work with lots of freedom and in my own style (not often taking notes in the lab books). The years working with you will undoubtedly still benefit my future career.

I would like to thank Altaf Hussain for helping me integrate into the CVENT project during the very first days of my research. Your help really paved the way to my achievements in this project. I am also greatly thankful to all partners of the project (ESAOTE Europe, Eindhoven University of Technology, Ruhr-University Bochum, Hôpital Européen Georges Pompidou, Lumibird, University of Bern, BrightLoop, SILIOS Technologies, and tp21) for sharing the knowledge and the collaborative work.

I would like to send my sincere thanks to the BMPI staffs. Thank you Sylvia for the magic that every time I came to you with an "I have a problem", I always got a "Problem solved!" from you plus a smile. Wilma, thanks for all nice recipes for cooking the chemicals in the lab and also for the friendly chats. Thank you Johan for reminding me to wear goggles, for reviving my translation stages and for a fancy probe holder design. And you Tom, thanks for letting me bother you with my issues like making a new water tank, 3D printing, ordering stuff. I really appreciate your enthusiasm.

I am greatly thankful to Prof. Srirang Manohar for his inputs and fruitful discussions. Thank you Yoeri for your idea to speed up the 3D back-projection. I really enjoyed our deep 
and interesting discussion on the back-projection and delay-and-sum algorithms with you and Srirang. David Thompson, thanks for proofreading my very first research article. My writing improved a lot from your comments. Sjoukje and Maura, thank you for your contribution to Chapter 9 of this thesis.

My special thanks to Hans-Jan, my bachelor student, for his hard work. I wish we had more time or continue someday to dive deeper into the work.

During the years at BMPI, I have been working in a very nice office. People might look at it differently but for me it has been more than an office. Ata, you became my first officemate as well as my first BMPI friend when I came to BMPI. Thank you for being a good friend, officemate, and practicummate. Colin, thank you for all the "conversations" over the years and your help with my Dutch and Twents. You were the only one I could talk with about football at BMPI. Thank you Carlos, Bahareh, and Giulia for building up a nice atmosphere in the office.

It was my pleasure to be part of the BMPI group. Thank you everyone for the discussions in the group meetings, for the chats and board games during the coffee breaks, for the tosti days, for the group events, and for the great memories over the years.

I am extremely grateful to my family and relatives for their unconditional and endless support and encouragement, especially to my Mom. Cảm ơn mẹ đã luôn động viên và lo lắng cho con. Special thanks to my brother's little family. No need to go to a vacation spot, your place is where I want to visit when I need a break from work. Playing with my nephew and niece (Pi and Hannah) makes me feel so refreshed after work. My girlfriend, thank you for coming into my life. It is so peaceful and relaxing when you are around. Especially the days that I was so busy with the manuscripts, having you around was like "Anh cứ làm việc đi, thế giới để em lo".

I would like to thank my Vietnamese friends in Enschede. Cảm ơn gia đình anh Đức, gia đình anh Nhân, gia đình anh Minh, gia đình anh Cường, gia đình chị Lan Anh, anh Hải chị Hòa, anh Tùng, Thành, Duy, Việt Anh cho những buổi liên hoan ấm áp, đậm vị Việt Nam và những buổi thể thao chém gió vui vẻ. 
I would also like to acknowledge the members of my graduation committee, Prof. dr. S. Manohar, Prof. dr. ir. R.M. Verdaasdonk, Prof. dr. ing. G. Schmitz, Dr. ir. R.G.P. Lopata, and Dr. M.K.A. Singh, for spending time and efforts on reviewing this thesis.

Enschede, October 2020 Như Ý 